\title{
Initial-boundary value problems for discrete evolution equations: discrete linear Schrödinger and integrable discrete nonlinear Schrödinger equations
}

\author{
Gino Biondini and Guenbo Hwang \\ State University of New York at Buffalo, Department of Mathematics, Buffalo, NY 14260
}

\begin{abstract}
We present a method to solve initial-boundary value problems for linear and integrable nonlinear differential-difference evolution equations. The method is the discrete version of the one developed by A. S. Fokas to solve initial-boundary value problems for linear and integrable nonlinear partial differential equations via an extension of the inverse scattering transform. The method takes advantage of the Lax pair formulation for both linear and nonlinear equations, and is based on the simultaneous spectral analysis of both parts of the Lax pair. A key role is also played by the global algebraic relation that couples all known and unknown boundary values. Even though additional technical complications arise in discrete problems compared to continuum ones, we show that a similar approach can also solve initial-boundary value problems for linear and integrable nonlinear differential-difference equations. We demonstrate the method by solving initial-boundary value problems for the discrete analogue of both the linear and the nonlinear Schrödinger equations, comparing the solution to those of the corresponding continuum problems. In the linear case we also explicitly discuss Robin-type boundary conditions not solvable by Fourier series. In the nonlinear case we also identify the linearizable boundary conditions, we discuss the elimination of the unknown boundary datum, we obtain explicitly the linear and continuum limit of the solution, and we write down the soliton solutions.
\end{abstract}

15 March 2022

\section{Introduction and outline}

The development of the theory of infinite-dimensional integrable systems was a remarkable advance of mathematical physics over the last forty years. One of the key properties of such systems is that they can be written as the compatibility condition of an overdetermined linear system, called the Lax pair. In turn, the existence of Lax pair is deeply related to many other features of these systems. Among them is the inverse scattering transform (IST), a nonlinear analogue of the Fourier transform which can be used to solve the initial value problem (IVP). The IST was successfully used in the late 1960's and early 1970's to solve IVPs on infinite domains or with periodic or quasi-periodic boundary conditions (BCs) for a variety of nonlinear partial differential equations (PDEs), differential-difference fully discrete, integro-differential equations, etc. (e.g., see Refs. [2, 7, 10, 19] and references therein).

Following the solution of IVPs, a natural issue was the solution of initial-boundary value problems (IBVPs). After some early results [8, 13, 14, 22], however, the issue remained essentially open for over twenty years. Recently, renewed interest in the problem has lead to a number of developments (e.g., see Refs. [9, 11, 12, 15, 23-30, 39-41] and references therein). Particularly important among these is the method developed by A. S. Fokas [23-30]. Fokas' method, which is a significant extension of the IST, is based on the simultaneous spectral analysis of both parts of the Lax pair. A crucial role is also played by a relation called 
global algebraic relation that couples all known and unknown boundary values. Indeed, it is the analysis of the global relation that allows one to express the unknown boundary datum in terms of known ones plus the initial datum. Importantly, the method also yields a new approach to IBVPs for linear PDEs, which allows the solution of new kinds of problems.

At the same time, the effort to extend the properties of integrable nonlinear PDEs to discrete integrable systems has been an ongoing theme in the last thirty years (e.g., see Refs. [2, 3, 5, 6, 20, 21, 31, 35, 36, 42] and references therein). The purpose of this work is to show that, mutatis mutandis, an approach similar to that for PDEs can also be used to solve IBVPs for linear and integrable nonlinear differential-difference equations (DDEs). We demonstrate this claim by solving IBVPs for the discrete analogue of the linear and nonlinear Schrödinger equations on the natural numbers. Note that the integrable discrete nonlinear Schrödinger (IDNLS) equation is an important model since it arises in a number of physical and mathematical contexts (e.g., see references in Ref. [6]).

The outline of this work is the following. In section 2 we solve the IBVP on the natural numbers for the discrete linear Schrödinger (DLS) equation, namely the linear DDE

$$
i \dot{q}_{n}+\frac{q_{n+1}-2 q_{n}+q_{n-1}}{h^{2}}=0
$$

where $q_{n}=q_{n}(t) \in \mathbb{C}, n \in \mathbb{N}, \dot{f} \equiv d f / d t$ denotes time derivative and $h$ is the lattice spacing. Then, in sections 3 and 4 we consider the IBVP for the integrable nonlinear counterpart of (1.1), namely the IDNLS equation or Ablowitz-Ladik (AL) equation [4, 5],

$$
i \dot{q}_{n}+\frac{q_{n+1}-2 q_{n}+q_{n-1}}{h^{2}}-v\left|q_{n}\right|^{2}\left(q_{n+1}+q_{n-1}\right)=0
$$

(where as usual the cases $v=-1$ and $v=1$ will be called respectively focusing and defocusing). In particular, in section 4 we discuss the elimination of the unknown boundary datum, the linearizable boundary conditions, and we write down the soliton solutions. Finally, in order to appreciate the similarities and differences between the method in the discrete versus the continuum case, in section 5 we review the solution of IBVPs for the continuum limits of both equations, namely the linear and nonlinear Schrödinger equations, and we discuss explicitly the correspondence between the method in the discrete case versus the continuum limit. The proof of various statements in the text is confined to the Appendix, which also contains a list of notations and frequently used formulae.

In both the linear and the nonlinear problem we will require the initial datum to be absolutely summable and the boundary datum $q_{0}(t)$ to be smooth, even though the method can be formulated under weaker conditions. The constant $h$ can be eliminated from (1.1) and (1.2) via the rescalings $t^{\prime}=t / h^{2}$ and $q_{n}^{\prime}(t)=h q_{n}(t)$. Thus, for simplicity we will consider the rescaled problems throughout (thus effectively setting $h=1$ ); however, we will will omit the primes except when considering the limit $h \rightarrow 0$ to recover the solution of the continuum cases. The indended meaning should be clear from the context. Also, for brevity we will occasionally omit functional dependences when doing so does not cause ambiguity.

\section{Discrete linear Schrödinger equation}

Here we solve the linear problem (1.1), which serves to introduce some of the tools that will be used in the nonlinear case. In section 2.1 we derive a Lax pair for (1.1). Then, in section 2.2 we solve the IVP and in section 2.3 IBVPs via spectral methods. 
IVP and IBVP for DLS via Fourier methods. Let us briefly review the solution of the IVP and the IBVP via Fourier methods. Doing so we will serve to introduce quantities that will also be used later. Consider first the IVP, namely (1.1) with $n \in \mathbb{Z}$ and with $q_{n}(0)$ given. We require that the initial datum $q_{n}(0)$ decays rapidly enough as $n \rightarrow \pm \infty$ to belong to $\ell^{1}(\mathbb{Z})$, the space of sequences $\left\{a_{n}\right\}_{n \in \mathbb{Z}}$ such that $\sum_{n=-\infty}^{\infty}\left|a_{n}\right|<\infty$. Introduce the transform pair as

$$
\begin{aligned}
& \hat{q}(k, t)=\sum_{n=-\infty}^{\infty} q_{n}(t) / z^{n}=\sum_{n=-\infty}^{\infty} \mathrm{e}^{-i n k} q_{n}(t), \\
& q_{n}(t)=\frac{1}{2 \pi i} \oint_{|z|=1} z^{n-1} \hat{q}(z, t) \mathrm{d} z=\frac{1}{2 \pi} \int_{-\pi}^{\pi} \mathrm{e}^{i n k} \hat{q}(k, t) \mathrm{d} k,
\end{aligned}
$$

where $z=\mathrm{e}^{i k}$, and the contour $|z|=1$ is oriented counterclockwise. The transformation $k \rightarrow z$ maps $k \in \mathbb{R}$ into $|z|=1$ and $\operatorname{Im} k \gtrless 0$ into $|z| \lesseqgtr 1$ (with $k= \pm i \infty$ corresponding respectively to $z=0$ and $z=\infty$ ). Use of (2.1) yields the solution of the IVP in Ehrenpreis form as

$$
q_{n}(t)=\frac{1}{2 \pi i} \oint_{|z|=1} z^{n-1} \mathrm{e}^{-i \omega(z) t} \hat{q}(z, 0) \mathrm{d} z=\frac{1}{2 \pi} \int_{-\pi}^{\pi} \mathrm{e}^{i(n k-\omega(k) t)} \hat{q}(k, 0) \mathrm{d} k,
$$

where the linear dispersion relation is

$$
\omega(z)=2-(z+1 / z)=2(1-\cos k) .
$$

Now consider the IBVP, namely (1.1) with $n \in \mathbb{N}$ and $t \in \mathbb{R}^{+}$, with $q_{n}(0)$ and $q_{0}(t)$ given. We assume $q_{n}(0) \in \ell^{1}(\mathbb{N})$ and $q_{0}(t) \in C\left(\mathbb{R}_{0}^{+}\right)$. Introduce the Fourier sine series and its inverse as

$$
\hat{q}^{(s)}(z, t)=\sum_{n=1}^{\infty} q_{n}(t)\left(1 / z^{n}-z^{n}\right), \quad q_{n}(t)=\frac{1}{4 \pi i} \oint_{|z|=1}\left(z^{n}-1 / z^{n}\right) \hat{q}^{(s)}(z, t) \mathrm{d} z / z,
$$

Use of this pair yields the solution of the IBVP as

$q_{n}(t)=\frac{1}{4 \pi i} \oint_{|z|=1}\left(z^{n}-1 / z^{n}\right) / z \mathrm{e}^{-i \omega(z) t} \hat{q}^{(s)}(z, 0) \mathrm{d} z-\frac{1}{4 \pi} \oint_{|z|=1}\left(z^{n}-1 / z^{n}\right) / z \mathrm{e}^{-i \omega(z) t} \hat{g}(z, t) \mathrm{d} z$,

where

$$
\hat{g}(z, t)=(z-1 / z) \int_{0}^{t} \mathrm{e}^{i \omega(z) t^{\prime}} q_{0}\left(t^{\prime}\right) \mathrm{d} t^{\prime}
$$

\subsection{A Lax pair for the discrete linear Schrödinger equation}

A Lax pair formulation, first discovered for nonlinear PDEs [33], is also possible for linear PDEs, and in fact it is the key to solving a wide class of IBVPs [24, 30]. Here we show how a Lax pair for the DLS equation (1.1). can be obtained by taking the linear limit of the the Lax pair of the IDNLS equation (1.2). (As in the continuum limit, an algorithmic way also exists to obtain the Lax pair associated to any linear discrete evolution equation. The corresponding formalism will be presented elsewhere.)

It is well-known that the IDNLS (1.2) is a reduction of the Ablowitz-Ladik (AL) system (A.4) [5]. A Lax pair for (A.4) is given by the overdetermined linear system (A.5). To obtain the linear limit of (A.5), let $\mathrm{Q}_{n}=O(\varepsilon)$, and take $\Phi_{n}(z, t)=\mathbf{v}_{n}(z, t)=\left(v_{1, n}, v_{2, n}\right)^{t}$ to be a two-component vector. The leading order solution of (A.5) is then $\mathbf{v}_{n}(z, t)=Z^{n} \mathrm{e}^{i(z-1 / z)^{2} \sigma_{3} t / 2} \mathbf{v}_{o}$, where $\mathbf{v}_{o}=\left(v_{1, o}, v_{2, o}\right)^{t}$ is an arbitrary constant vector. Choosing $v_{2, o}=1$ and keeping terms up to $O(\varepsilon)$ then yields the following scalar linear system for $v_{1, n}$ :

$$
\begin{aligned}
& v_{1, n+1}-z v_{1, n}=q_{n} z^{-n} \mathrm{e}^{-i(z-1 / z)^{2} t / 2} \\
& \dot{v}_{1, n}-\frac{i}{2}(z-1 / z)^{2} v_{1, n}=i\left(z q_{n}-q_{n-1} / z\right) z^{-n} \mathrm{e}^{-i(z-1 / z)^{2} t / 2} .
\end{aligned}
$$


Enforcing the compatibility of (2.5a) and (2.5b) now yields the discrete linear Schrödinger equation (1.1). To eliminate the dependence on $z^{n}$ from the right-hand side (RHS) of (2.5a), we now perform the rescaling $z^{\prime}=z^{2}$ and $\phi_{n}=z^{n-1} \mathrm{e}^{i(z-1 / z)^{2} t / 2} v_{1, n}$. Dropping primes for simplicity, we then obtain the following Lax pair for (1.1):

$$
\phi_{n+1}-z \phi_{n}=q_{n}, \quad \dot{\phi}_{n}+i \omega(z) \phi_{n}=i\left(q_{n}-q_{n-1} / z\right),
$$

where $\omega(z)$ is given by (2.3) as before. Indeed, although it may not be obvious at this point, the meaning of the variable $z$ in (2.6) coincides exactly with that of $z$ in (2.1).

The rescaling $z^{\prime}=z^{2}$ between the linear and the nonlinear problem is the discrete analogue of the rescaling $k^{\prime}=2 k$ in the continuum limit. Such rescaling will reflect on the location of the jumps in the Riemann-Hilbert problem (RHP) for the IBVP in the nonlinear problem, which will differ from the corresponding locations in the linear problem.

\subsection{IVP for DLS via spectral analysis of the Lax pair}

We now solve the IVP for (1.1) using spectral methods. Doing so will introduce some of the ideas that will be useful for the IBVP and nonlinear case. Making use of the integrating factor $z^{n} \mathrm{e}^{-i \omega(z) t}$ [with $\omega(z)$ as in (2.3)], we introduce the modified eigenfunction

$$
\psi_{n}(z, t)=z^{-n} e^{i \omega(z) t} \phi_{n}(z, t)
$$

which satisfies the following modified Lax pair:

$$
\psi_{n+1}-\psi_{n}=\mathrm{e}^{i \omega(z) t} q_{n} / z^{n+1}, \quad \dot{\psi}_{n}=\mathrm{e}^{i \omega(z) t} i\left(q_{n}-q_{n-1} / z\right) / z^{n}
$$

Of course the above linear system is also compatible if $q_{n}(t)$ satisfies (1.1). It is then easy to define $\phi_{n}^{(1,2)}(z, t)$ as the solutions of (2.6) which vanish as $n \rightarrow \mp \infty$, respectively:

$$
\phi_{n}^{(1)}(z, t)=\sum_{m=-\infty}^{n-1} q_{m}(t) z^{n-m-1}, \quad \phi_{n}^{(2)}(z, t)=-\sum_{m=n}^{\infty} q_{m}(t) z^{n-m-1} .
$$

Note that $\phi_{n}^{(1)}(z, t)$ is analytic as a function of $z$ for $|z|<1$ and continuous on $|z|=1$, while $\phi_{n}^{(2)}(z, t)$ is analytic for $|z|>1$ and bounded for $|z|=1$. The jump conditions obtained by evaluating $\phi_{n}^{(1,2)}(z, t)$ on $|z|=1$ then yield a scalar RHP: $\phi_{n}^{(1)}(z, t)-\phi_{n}^{(2)}(z, t)=z^{n-1} \hat{q}(z, t)$, where $\hat{q}(z, t)$ is given by (2.1). However, the difference $\phi_{n}^{(1)}-\phi_{n}^{(2)}$ solves the homogeneous version of (2.6), and hence it depends on $n$ and $t$ only through the factor $z^{n} \mathrm{e}^{-i \omega(z) t}$. Evaluating (2.9) at $(n, t)=(0,0)$ we can then rewrite the jump condition as:

$$
\phi_{n}^{(1)}(z, t)-\phi_{n}^{(2)}(z, t)=z^{n-1} \mathrm{e}^{-i \omega(z) t} \hat{q}(z, 0), \quad|z|=1 .
$$

Equations (2.9) imply $\phi_{n}^{(1)}(0, t)=q_{n-1}(t) \neq 0$, and $\phi_{n}^{(2)}(z, t) \rightarrow 0$ as $z \rightarrow \infty$. Thus, the RHP defined by (2.10) is trivially solved by applying standard Cauchy projectors, namely:

$$
\phi_{n}(z, t)=\frac{1}{2 \pi i} \oint_{|\zeta|=1} \zeta^{n-1} \mathrm{e}^{-i \omega(\zeta) t} \frac{\hat{q}(\zeta, 0)}{\zeta-z} \mathrm{~d} \zeta,
$$

where the contour is oriented counterclockwise, as usual. Then, inserting (2.11) into the LHS of the first of (2.6), one obtains the solution of the IVP as (2.2).

The continuum limit of (2.2) yields the solution of the linear Schrödinger equation. Indeed, reinstating the lattice spacing $h$, the solution of the IVP for the DLS (2.2) is

$$
q_{n}(t)=\frac{1}{2 \pi} \int_{-\pi / h}^{\pi / h} \mathrm{e}^{i(n k h-\omega(k) t)} \hat{q}(k, 0) \mathrm{d} k,
$$

where now $\omega(k)=2(1-\cos k h) / h^{2}$ and

$$
\hat{q}(k, t)=h \sum_{n=-\infty}^{\infty} \mathrm{e}^{-i n k h} q_{n}(t) .
$$

Then, taking the limit $h \rightarrow 0$ of (2.12) with $x_{n}=n h$ fixed, one obtains (5.3) and the first of (5.2). 

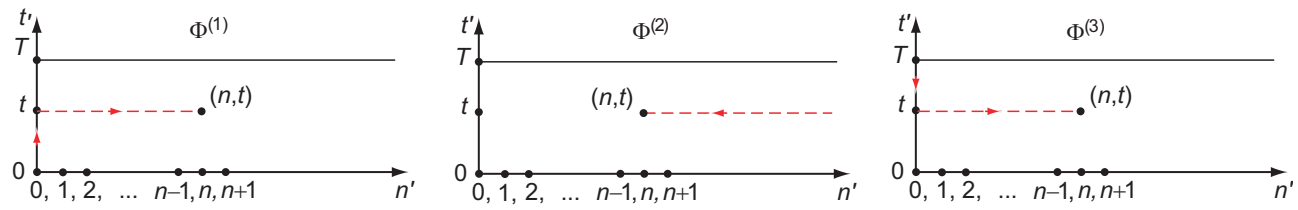

Figure 1. The distinguished points for the eigenfunctions $\phi_{n}^{(1)}, \phi_{n}^{(2)}$ and $\phi_{n}^{(3)}$.

\subsection{IBVP for DLS via spectral analysis of the Lax pair}

We now use spectral methods to solve the IBVP for the DLS, namely (1.1) for $n \in \mathbb{N}$ and $t \in \mathbb{R}^{+}$, with $q_{n}(0)$ and $q_{0}(t)$ given, where as before we assume $q_{n}(0) \in \ell^{1}(\mathbb{N})$ and $q_{0}(t) \in C\left(\mathbb{R}_{0}^{+}\right)$. Before we do so, however, we address the issue of the well-posedness of the linear system (2.6).

In the continuum limit, the $t$-part of the Lax pair evaluated at $x=0$ depends on $q(0, t)$ and $q_{x}(0, t)$, only one of which is given. Use of the global relation allows one to obtain the unknown $\mathrm{BC}$ in terms of the given one. In the discrete case, evaluation of the $t$-part of the Lax pair for $n=0$ requires the knowledge of $q_{-1}(t)$. Thus, the role of the unknown boundary datum in the discrete case is played by the fictitious function $q_{-1}(t)$. In analogy with the continuum limit, the solution method proceeds as though this function is given; a posteriori we will then show that this unknown boundary datum is determined in terms of known initial-boundary data via the global relation.

A similar problem arises with Fourier methods, where one must define an appropriate transform so that the unknown boundary data do not appear in the expression for the solution. A similar situation also occurs in IBVPs for Burgers' equation [13, 14], where the solution depends on an unknown function that must be determined a posteriori. There, similarly to nonlinear PDEs solvable by the IST, the IBVP is reduced to a nonlinear integro-differential equation [14], which can be linearized for special kinds of BCs [13].

Eigenfunctions and analyticity. As in the continuum case [24, 26, 28], to solve the IBVP we consider simultaneous solutions of both the $x$-part and the $t$-part of the Lax pair. To do this we again use $\psi_{n}(z, t)$, defined in (2.7). Integrating (2.8), we then define three eigenfunctions uniquely determined in terms of their normalizations: namely, $\phi_{n}^{(j)}(z, t)$ for $j=1,2,3$, so that $\phi_{n}^{(j)}(z, t)=0$ respectively at $(n, t)=(0,0)$, as $(n, t) \rightarrow(\infty, t)$ and at $(n, t)=(0, T)$ (cf. Fig. 1):

$$
\begin{aligned}
& \phi_{n}^{(1)}(z, t)=\sum_{m=0}^{n-1} q_{m}(t) z^{n-m-1}+i z^{n} \int_{0}^{t} \mathrm{e}^{-i \omega(z)\left(t-t^{\prime}\right)}\left(q_{0}\left(t^{\prime}\right)-q_{-1}\left(t^{\prime}\right) / z\right) \mathrm{d} t^{\prime}, \\
& \phi_{n}^{(2)}(z, t)=-\sum_{m=n}^{\infty} q_{m}(t) z^{n-m-1}, \\
& \phi_{n}^{(3)}(z, t)=\sum_{m=0}^{n-1} q_{m}(t) z^{n-m-1}-i z^{n} \int_{t}^{T} \mathrm{e}^{-i \omega(z)\left(t-t^{\prime}\right)}\left(q_{0}\left(t^{\prime}\right)-q_{-1}\left(t^{\prime}\right) / z\right) \mathrm{d} t^{\prime} .
\end{aligned}
$$

We introduce the domains $D_{ \pm}=\{z \in \mathbb{C}: \operatorname{Im} \omega(z) \gtrless 0\}$, which will also be convenient to decompose as $D_{ \pm}=D_{ \pm \text {in }} \cup D_{ \pm \text {out }}$, where $D_{ \pm \text {in }}$ and $D_{ \pm \text {out }}$ are respectively the portions of $D_{ \pm}$ inside and outside the unit disk (cf. Fig. 2), namely

$$
\begin{array}{ll}
D_{\text {+in }}=\{z \in \mathbb{C}:|z|<1 \wedge \operatorname{Im} z>0\}, & D_{\text {+out }}=\{z \in \mathbb{C}:|z|>1 \wedge \operatorname{Im} z<0\}, \\
D_{- \text {in }}=\{z \in \mathbb{C}:|z|<1 \wedge \operatorname{Im} z<0\}, & D_{\text {-out }}=\{z \in \mathbb{C}:|z|>1 \wedge \operatorname{Im} z>0\} .
\end{array}
$$

We then note that: 

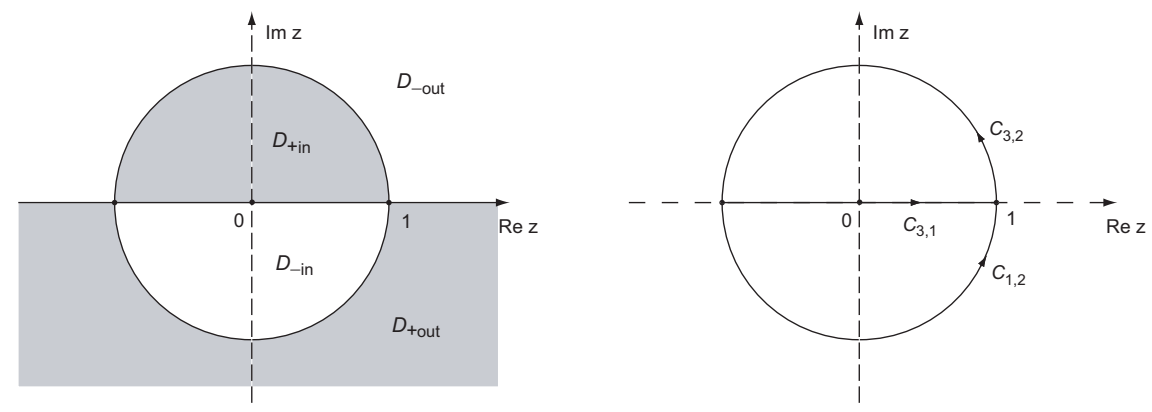

Figure 2. (Left) The regions $D_{+}$(shaded) and $D_{-}$(white) of the $z$-plane where $\operatorname{Im}[\omega(z)] \gtrless 0$. (Right) The contours $C_{1,2}, C_{2,3}$ and $C_{3,1}$ that define the Riemann-Hilbert problem in the linear case (see text for details).

- $\phi_{n}^{(2)}$ coincides with the eigenfunction in the IVP, hence it is analytic for $|z|>1$ and continuous and bounded for $|z| \geq 1$, and $\phi_{n}^{(2)}(z, t) \rightarrow 0$ as $z \rightarrow \infty$;

- $\phi_{n}^{(1)}$ and $\phi_{n}^{(3)}$ are analytic in the punctured complex $z$-plane $\mathbb{C}^{[\emptyset]}$;

- for all $t>0$ it is $\mathrm{e}^{i \omega(z) t} \rightarrow 0$ as $z \rightarrow 0, \infty$ in $D_{+}$and $\mathrm{e}^{-i \omega(z) t} \rightarrow 0$ as $z \rightarrow 0, \infty$ in $D_{-}$; as a result, $\phi_{n}^{(1)}$ and $\phi_{n}^{(3)}$ are bounded respectively for $z \in \bar{D}_{- \text {in }}$ and $z \in \bar{D}_{+ \text {in }}$.

Note that (2.13) do not define $\phi_{0}^{(1)}(z, t)$ and $\phi_{0}^{(3)}(z, t)$ at $z=0$. In Appendix D, however, we compute the asymptotics of these eigenfunctions as $z \rightarrow 0$, and we show that $\phi_{0}^{(1)}(z, t)=O(1)$ as $z \rightarrow 0$ with $\operatorname{Im} z \leq 0$ and $\phi_{0}^{(3)}(z, t)=O(1)$ as $z \rightarrow 0$ with $\operatorname{Im} z \geq 0$.

Jump conditions and Riemann-Hilbert problem. The difference between eigenfunctions at $|z|=1$ and $z \in[-1,1]$ yields a scalar RHP whose solution will enable us to reconstruct the potential in terms of the scattering data. As before, the difference between any eigenfunctions solves the homogeneous version of (2.6). Evaluating these differences at $(n, t)=(0,0)$ we then obtain the jumps as (of course any two of the jumps uniquely determine the third one):

$\phi_{n}^{(1)}(z, t)-\phi_{n}^{(2)}(z, t)=z^{n-1} \mathrm{e}^{-i \omega(z) t} \hat{q}(z, 0)$

$|z|=1 \wedge \operatorname{Im} z \leq 0$

$\phi_{n}^{(1)}(z, t)-\phi_{n}^{(3)}(z, t)=z^{n-1} \mathrm{e}^{-i \omega(z) t} \hat{F}(z, T)$

$\phi_{n}^{(3)}(z, t)-\phi_{n}^{(2)}(z, t)=z^{n-1} \mathrm{e}^{-i \omega(z) t}(\hat{q}(z, 0)-\hat{F}(z, T)), \quad|z|=1 \wedge \operatorname{Im} z \geq 0$,

with $\hat{F}(z, t)=i\left(z \hat{f}_{0}(z, t)-\hat{f}_{-1}(z, t)\right)$, and where $\hat{q}(z, t)$ and $\hat{f}_{n}(z, t)$ are respectively the $z$ transforms of the initial and boundary data; namely:

$$
\hat{q}(z, t)=\sum_{m=0}^{\infty} q_{m}(t) / z^{m}, \quad \hat{f}_{n}(z, t)=\int_{0}^{t} \mathrm{e}^{i \omega(z) t^{\prime}} q_{n}\left(t^{\prime}\right) \mathrm{d} t^{\prime} .
$$

Note that $\hat{q}(z, t)$ is analytic for $|z|>1$ and continuous and bounded for $|z| \geq 1$, while the $\hat{f}_{n}(z, t)$ are analytic $\forall z \neq 0$ and continuous and bounded for $z \in \bar{D}_{+}$. Moreover, $\hat{q}(z, t) \rightarrow q_{0}(t)$ as $z \rightarrow \infty$, while $\hat{f}_{n}(z, t) \rightarrow 0$ as $z \rightarrow 0, \infty$ in $D_{+}$. Finally, integration by parts shows that

$$
\hat{f}_{n}(z, t)=i z\left(\mathrm{e}^{i \omega(z) t} q_{n}(t)-q_{n}(0)\right)+O\left(z^{2}\right)
$$

as $z \rightarrow 0$ in $\partial D_{+}$(i.e., along the real $z$-axis). As shown in Appendix B, (2.14) are inverted by

$$
q_{n}(t)=\frac{1}{2 \pi i} \oint_{|z|=1} z^{n-1} \hat{q}(z, t) \mathrm{d} z, \quad q_{n}(t)=\frac{1}{2 \pi} \int_{\partial D_{\text {+out }}} \omega^{\prime}(z) \mathrm{e}^{-i \omega(z) t} \hat{f}_{n}(z, T) \mathrm{d} z,
$$


for all $0<t<T$, where $\omega^{\prime}(z)=d \omega / d z$ and $\partial D_{+ \text {out }}$ is oriented so that $\operatorname{Re} z$ is decreasing.

Note that $\hat{F}(z, T) / z$ remains bounded as $z \rightarrow 0$ along the real $z$-axis [cf. Appendix D]. Thus, the RHS of (2.14b) with $n=0$ does not have a pole at $z=0$. The solution of the RHP defined by (2.14) is therefore simply obtained using standard Cauchy projectors over the unit circle:

$$
\phi_{n}(z, t)=\frac{1}{2 \pi i} \oint_{|\zeta|=1} \zeta^{n-1} \mathrm{e}^{-i \omega(\zeta) t} \frac{\hat{q}(\zeta, 0)}{\zeta-z} \mathrm{~d} \zeta-\frac{1}{2 \pi i} \oint_{\partial D_{+ \text {in }}} \zeta^{n-1} \mathrm{e}^{-i \omega(\zeta) t} \frac{\hat{F}(\zeta, T)}{\zeta-z} \mathrm{~d} \zeta,
$$

where $|\zeta|=1$ is taken counterclockwise and $\partial D_{+}$is oriented so as to leave the domain to its left, as usual. Inserting (2.18) into the first of (2.6) then yields the reconstruction formula:

$$
q_{n}(t)=\frac{1}{2 \pi i} \oint_{|z|=1} z^{n-1} \mathrm{e}^{-i \omega(z) t} \hat{q}(z, 0) \mathrm{d} z-\frac{1}{2 \pi i} \int_{\partial D_{+\mathrm{in}}} z^{n-1} \mathrm{e}^{-i \omega(z) t} \hat{F}(z, T) \mathrm{d} z
$$

Of course the right-hand side of (2.19) still depends on the undetermined value $q_{-1}(t)$ via its transform $\hat{f}_{-1}(z, T)$. We next show how to eliminate this unknown using the global relation.

Global relation and symmetries. The global relation, which couples all initial and boundary values, is obtained in a similar way as in the continuum problem by integrating (2.8) around the edges of the domain $\mathbb{N}_{0} \times[0, T]$, namely for $(n, t)$ from $(0,0)$ to $(0, T)$, from there to $(\infty, T)$, and then to $(\infty, 0)$ and back to $(0,0)$ :

$$
i \int_{0}^{t} \mathrm{e}^{i \omega(z) t^{\prime}}\left(q_{0}\left(t^{\prime}\right)-q_{-1}\left(t^{\prime}\right) / z\right) \mathrm{d} t^{\prime}+\mathrm{e}^{i \omega(z) t} \sum_{m=0}^{\infty} q_{m}(t) / z^{m+1}=\sum_{m=0}^{\infty} q_{m}(0) / z^{m+1} .
$$

Equation (2.20) holds where all of its terms are defined, that is, for all $|z| \geq 1$. In terms of the $z$-transforms:

$$
i\left[z \hat{f}_{0}(z, t)-\hat{f}_{-1}(z, t)\right]+\mathrm{e}^{i \omega(z) t} \hat{q}(z, t)=\hat{q}(z, 0) .
$$

Now note that $\omega(z)$ is invariant under the transformation $z \rightarrow 1 / z$, and therefore so are the functions $\hat{f}_{n}(z, t)$. Moreover, $z \in D_{\text {+out }}$ implies $1 / z \in D_{+ \text {in }}$ and viceversa. Hence, (2.21) with $z \rightarrow 1 / z$ gives, for all $0<|z| \leq 1$ :

$$
i\left[(1 / z) \hat{f}_{0}(z, t)-\hat{f}_{-1}(z, t)\right]+\mathrm{e}^{i \omega(z) t} \hat{q}(1 / z, t)=\hat{q}(1 / z, 0) .
$$

We can then solve for $\hat{f}_{-1}(z, t)$, obtaining, for all $0<|z| \leq 1$ :

$$
\hat{f}_{-1}(z, t)=\hat{f}_{0}(z, t) / z-i\left(\mathrm{e}^{i \omega(z) t} \hat{q}(1 / z, t)-\hat{q}(1 / z, 0)\right) .
$$

Solution of the IBVP. Of course the RHS of (2.23) contains $\mathrm{e}^{i \omega(z) T} \hat{q}(1 / z, T)$, which is (apart from the changes $t \rightarrow T$ and $z \rightarrow 1 / z)$ the transform of the solution we are trying to recover. When this terms is inserted in (2.19), however, the resulting integrand is $z^{n-1} \mathrm{e}^{i \omega(z)(T-t)} \hat{q}(1 / z, t)$, which is analytic and bounded in $D_{+ \text {in }}$, and whose integral over $\partial D_{+ \text {in }}$ is therefore zero. [This is analogous to what happens in the continuum limit; cf. section 5.] Importantly, the result also holds for $n=0$, since $\mathrm{e}^{i \omega(z)(T-t)}$ decays exponentially for all $t<T$ as $z \rightarrow 0$ in $D_{+ \text {in }}$. We then have

$q_{n}(t)=\frac{1}{2 \pi i} \oint_{|z|=1} z^{n-1} \mathrm{e}^{-i \omega(z) t} \hat{q}(z, 0) \mathrm{d} z+\frac{1}{2 \pi} \int_{\partial D_{+ \text {in }}} z^{n-1} \mathrm{e}^{-i \omega(z) t}\left[i \hat{q}(1 / z, 0)-(z-1 / z) \hat{f}_{0}(z, T)\right] \mathrm{d} z$.

Equation (2.24) provides the solution of the IBVP in Ehrenpreis form [18, 37, 32], since the only dependence of the RHS on $n$ and $t$ is via the terms $z^{n} \mathrm{e}^{-i \omega(z) t}$, as in the IVP. Performing the 
change of variable $z^{\prime}=1 / z$ we can write the second term in the RHS of (2.24) as an integral over $\partial D_{\text {+out }}$. Then, since the resulting integrand, $\mathrm{e}^{-i \omega(z) t} \hat{q}(z, 0) / z^{n+1}$ is analytic on $D_{- \text {out }}$, for that portion we can deform the contour $\partial D_{+ \text {out }}$ onto the circle $|z|=1$ and combine the result with the first integral in (2.24), obtaining the equivalent representation

$q_{n}(t)=\frac{1}{2 \pi i} \oint_{|z|=1}\left(z^{n}-z^{-n}\right) / z \mathrm{e}^{-i \omega(z) t} \hat{q}(z, 0) \mathrm{d} z-\frac{1}{2 \pi} \int_{\partial D_{\text {+out }}}(z-1 / z) z^{-n-1} \mathrm{e}^{-i \omega(z) t} \hat{f}_{0}(z, T) \mathrm{d} z$

where, as before, $\partial D_{+ \text {out }}$ is oriented so that $\operatorname{Re} z$ is decreasing.

Continuum limit. The representation (2.25) is the discrete analogue of the solution in the continuum limit. To see this, one can reinstate the lattice spacing $h$ and follow the same steps as above. When expressed in terms of $k$, the solution of the IBVP then becomes:

$$
q_{n}(t)=\frac{2}{\pi} \int_{0}^{\pi / h} \mathrm{e}^{-i \omega(k) t} \sin (n k h) \hat{q}^{\left(s^{\prime}\right)}(k, 0) \mathrm{d} k+\frac{1}{\pi} \int_{0}^{\pi / h} \mathrm{e}^{-i \omega(k) t} \sin (n k h) \hat{g}(k, t) \mathrm{d} k,
$$

where $\omega(k)=2(1-\cos (k h)) / h^{2}$, and with

$$
\hat{q}^{\left(s^{\prime}\right)}(k, t)=h \sum_{n=1}^{\infty} \sin (n k h) q_{n}(t), \quad \hat{g}(k, t)=2 i \frac{\sin (k h)}{h} \int_{0}^{t} \mathrm{e}^{i \omega(k) t^{\prime}} q_{0}\left(t^{\prime}\right) \mathrm{d} t^{\prime} .
$$

It is then trivial to show that, in the limit $h \rightarrow 0$, (2.26) yield the solution of the continuum problem, namely (5.5).

Remarks. Assuming existence, one can now verify that the RHS of (2.24) and (2.25) indeed satisfies the DDE as well as the initial and BCs. That the function defined by (2.24) solves the DLS equation is a trivial consequence of the fact that it is in Ehrenpreis form. When $t=0$ the term proportional to $z^{-n}$ in the first integral of (2.25) gives zero contribution, since the corresponding integrand is analytic, bounded for $|z|>1$, and $O\left(1 / z^{n+1}\right)$ as $z \rightarrow \infty$. Similarly, the second integral vanishes for the same reasons. The only piece left coincides with the RHS of the first of (2.17) at $t=0$, which therefore yields the initial datum $q_{n}(0)$. Finally, for $n=0$ the first integral in (2.25) is obviously zero, while the second becomes just the inversion integral in (2.17). Hence its result is simply $q_{0}(t)$.

Even though $\hat{f}_{0}(z, T)$ depends on values of the BC $q_{0}(t)$ at all times $t$ from 0 to $T$, in practice (2.25) preserves causality, and the solution of the IBVP at time $t$ does not depend on future values of the BCs, because one can replace $T$ with $t$ in (2.25). The reason is that the difference between the two terms is

$$
\frac{1}{2 \pi} \int_{\partial D_{\text {+out }}}(z-1 / z) z^{-n-1} \int_{t}^{T} \mathrm{e}^{-i \omega(z)\left(t-t^{\prime}\right)} q_{0}\left(t^{\prime}\right) \mathrm{d} t^{\prime} \mathrm{d} z
$$

and $\forall n \neq 0$ the integrand is analytic and bounded in $D_{+ \text {out }}$, and vanishes as $z \rightarrow \infty$ in $D_{+}$. Hence, the integral is zero $\forall n>0$.

For all $n \neq 0$, the second integrand in (2.25) is analytic and bounded in $D_{\text {-out }}$. Hence we can deform the integration contour from $\partial D_{+ \text {out }}$ to $|z|=1$, and substitute $z \rightarrow 1 / z$ in half of the integral. The resulting expression for the solution coincides with the solution of the IBVP via sine series, namely (2.4). We reiterate however that (2.25) also holds for $n=0$, unlike (2.4).

Unlike sine/cosine transforms, the present method works equally well for more general BCs, as we show below. Also, unlike sine/cosine transforms, the present method can solve IBVPs for arbitrary linear discrete evolution equations. Finally, the method can be generalized to solve IBVPs for integrable nonlinear DDEs, as we show in section 3. 
Other boundary conditions. We now consider a IBVP for the DLS equation (1.1) in which the BCs are a linear combination of $q_{0}(t)$ and $q_{-1}(t)$ with constant coefficients, namely, when

$$
q_{-1}(t)-\alpha q_{0}(t)=h(t)
$$

is given, $\alpha \in \mathbb{C}$ is a nonzero but otherwise arbitrary constant, and where in this case the labeling of the lattice should be such that $n=-1$, not $n=0$, is the first lattice site. Such BCs are the discrete analogue of Robin-type BCs in IBVPs for PDEs, and cannot be solved using sine/cosine series. The present method however works equally well; the only difference from the previous case being that one needs to solve the global relation for a different unknown. Indeed, in Appendix $\mathrm{C}$ we show that the solution of this IBVP is given by

$q_{n}(t)=\frac{1}{2 \pi i} \oint_{|z|=1} z^{n-1} \mathrm{e}^{-i \omega(z) t} \hat{q}(z, 0) \mathrm{d} z-\frac{1}{2 \pi i} \int_{\partial D_{+ \text {in }}} z^{n-1} \mathrm{e}^{-i \omega(z) t} \frac{\hat{G}(z, T)}{1 / z-\alpha} \mathrm{d} z-v_{\alpha} \alpha^{-n-1} \mathrm{e}^{-i \omega(\alpha) t} \hat{G}(1 / \alpha, t)$,

where

$$
\hat{G}(z, t)=i(z-1 / z) \hat{h}(z, t)+(z-\alpha) \hat{q}(1 / z, 0),
$$

and where $v_{\alpha}=1$ if $\alpha \in D_{+ \text {out }}, v_{\alpha}=1 / 2$ if $\alpha \in \partial D_{+ \text {out }}$ and $v_{\alpha}=0$ otherwise, and where the integral along $\partial D_{+ \text {in }}$ is to be taken in the principal value sense when $\alpha \in \partial D_{+ \text {out }}$. As before, one can easily verify that the expression in (2.28) indeed solves (1.1) and satisfies the initial condition and the BC (2.27). Moreover, one can also verify that, in the limit $\alpha \rightarrow \infty$ with $h(t) / \alpha=h^{\prime}(t)$ finite, the solution of the IBVP with "Dirichlet-type" BCs [namely (2.24)], is recovered.

\section{Integrable discrete nonlinear Schrödinger equation}

We now turn our attention to IVBPs for the IDNLS equation (1.2). As before, we first review the IVP, which serves to introduce some of tools that will be used for the IBVP. We require the same regularity conditions on the initial-boundary data as in the linear case.

\subsection{The Ablowitz-Ladik system on the integers}

Consider the AL system (A.4) with $n \in \mathbb{Z}$ and $t \in \mathbb{R}^{+}$, and with $q_{n}(0)$ given. A Lax pair for (A.4) is given by (A.5), where now we take $\Phi_{n}(z, t)$ to be a $2 \times 2$ matrix, $\mathrm{Q}_{n}(t)$ and $\mathrm{H}_{n}(z, t)$ are defined in (A.6), and $\omega(z) \equiv \omega_{\text {idnls }}(z)=\omega_{\text {dls }}\left(z^{2}\right) / 2$, where $\omega_{\text {dls }}(z)$ was defined in (2.3). As in the linear case, we assume $q_{n}(0) \in \ell^{1}(\mathbb{Z})$. (As in the continuum limit, the IST with non-vanishing BCs at infinity is significantly more involved, see Refs. [1, 43].)

Jost solutions. As customary, we remove the $n$-dependence of the eigenfunctions as $n \rightarrow \pm \infty$ by introducing a modified eigenfunction as

$$
\Phi_{n}(z, t)=\mu_{n}(z, t) Z^{n} \mathrm{e}^{-i \omega(z) t \sigma_{3}} .
$$

(This definition differs from the usual one by the factor $\mathrm{e}^{-i \omega(z) t \sigma_{3}}$, which has been added for consistency with the the IBVP, discussed in section 3.2. With this choice, the scattering matrix will be independent of time.) Then $\mu_{n}(z, t)$ satisfies the following modified Lax pair:

$$
\mu_{n+1}-\hat{Z} \mu_{n}=\mathrm{Q}_{n} \mu_{n} \mathrm{Z}^{-1}, \quad \dot{\mu}_{n}+i \omega(z)\left[\sigma_{3}, \mu_{n}\right]=\mathrm{H}_{n} \mu_{n},
$$

where $\hat{Z} A=Z A Z^{-1}$ It is also useful to use the integrating factor $e^{i \theta \hat{\sigma}_{3}}(A)=\mathrm{e}^{i \theta \sigma_{3}} \mathrm{Ae}^{-i \theta \sigma_{3}}$ (cf. Appendix A). Then, the function

$$
\Psi_{n}(z, t)=\hat{Z}^{-n} \mathrm{e}^{i \omega(z) t \hat{\sigma}_{3}} \mu_{n}(z, t),
$$


solves

$$
\Psi_{n+1}-\Psi_{n}=\mathrm{Z}^{-1} \hat{Z}^{-n} \mathrm{e}^{i \omega(z) t \hat{\sigma}_{3}}\left(\mathrm{Q}_{n}\right) \Psi_{n} . \quad \dot{\Psi}_{n}=\hat{Z}^{-n} \mathrm{e}^{i \omega(z) t \hat{\sigma}_{3}}\left(\mathrm{H}_{n}\right) \Psi_{n} .
$$

One can now easily "integrate" (3.4) and thereby obtain the solutions of (3.2) which reduce to the identity matrix as $n \rightarrow \mp \infty$ :

$\mu_{n}^{(1)}(z, t)=\mathrm{I}+\mathrm{Z}^{-1} \sum_{m=-\infty}^{n-1} \hat{Z}^{n-m}\left(\mathrm{Q}_{m} \mu_{m}^{(1)}\right), \quad \mu_{n}^{(2)}(z, t)=\mathrm{I}-\mathrm{Z}^{-1} \sum_{m=n}^{\infty} \hat{Z}^{n-m}\left(\mathrm{Q}_{m} \mu_{m}^{(2)}\right)$.

Of course, unlike the linear case the eigenfunctions are now defined in terms of summation equations (the discrete analogue of integral equations).

As in the linear problem, (3.5) imply certain analyticity properties for the eigenfunctions. More precisely, let $\mu_{n}^{(j)}(z, t)=\left(\mu_{n}^{(j, L)}, \mu_{n}^{(j, R)}\right), j=1,2$, where the column vectors $\mu_{n}^{(j, L)}(z, t)$ and $\mu_{n}^{(j, R)}(z, t)$ denote respectively the first and second column of $\mu_{n}^{(j)}(z, t)$. These columns are analytic in the following regions [6]:

$$
\mu_{n}^{(1, L)}, \mu_{n}^{(2, R)}: \quad|z|>1, \quad \mu_{n}^{(1, R)}, \mu_{n}^{(2, L)}: \quad|z|<1,
$$

Moreover, these columns are continuous and bounded on the closure of these domains. These properties immediately yield those of $\Phi_{n}^{(j)}(z, t)=\mu_{n}^{(j)}(z, t) Z^{n} \mathrm{e}^{-i \omega(z) t \sigma_{3}}$ for $j=1,2$ : $\Phi_{n}^{(1, L)}(z, t)$ and $\Phi_{n}^{(2, R)}(z, t)$ are analytic for $|z|>1$, and $\Phi_{n}^{(1, R)}(z, t)$ and $\Phi_{n}^{(2, L)}(z, t)$ for $|z|<1$.

Scattering matrix. Equation (A.5a) implies $\operatorname{det} \Phi_{n+1}=\left(1-q_{n} p_{n}\right) \operatorname{det} \Phi_{n}$. Therefore

$$
\operatorname{det} \Phi_{n}^{(1)}=\prod_{m=-\infty}^{n-1}\left(1-q_{m} p_{m}\right), \quad \operatorname{det} \Phi_{n}^{(2)}=\prod_{m=n}^{\infty}\left(1-q_{m} p_{m}\right)^{-1}=: 1 / C_{n} .
$$

(Note $\operatorname{det} \Phi_{n}=\operatorname{det} \mu_{n}$.) Equations (3.6) mark a significant difference of the discrete case from the continuum case, where such determinants are independent of both the potential and the independent variable (cf. section 5).

For the focusing IDNLS [namely, (A.4) with $p_{n}=v q_{n}^{*}$ and $v=-1$ ], $1-q_{n} p_{n}=1+\left|q_{n}\right|^{2}$, and therefore $\operatorname{det} \mu_{n}^{(j)} \neq 0 \forall n \in \mathbb{Z}$ for $j=1,2$. For the defocusing case $(v=1)$, however, it is necessary to assume that $\left|q_{n}\right| \neq 1 \forall n \in \mathbb{Z}$ in order that $\operatorname{det} \mu_{n}^{(j)}$ to be guaranteed to be nonzero. Hereafter we will assume that $q_{n} p_{n} \neq 1 \forall n \in \mathbb{Z}$. Moreover, we will require that the product

$$
C_{-\infty}=\operatorname{det} \Phi_{\infty}^{(1)}=1 / \operatorname{det} \Phi_{-\infty}^{(2)}=\prod_{n=-\infty}^{\infty}\left(1-q_{n} p_{n}\right)
$$

be finite, which will simplify the study of the scattering coefficients. Under these hypotheses, the matrices $\Phi_{n}^{(1)}$ and $\Phi_{n}^{(2)}$ are both fundamental solutions of the scattering problem (A.5a). Hence they must be proportional to each other: $\Phi_{n}^{(1)}(z, t)=\Phi_{n}^{(2)}(z, t) \mathrm{A}(z)$ on $|z|=1$, where $\mathrm{A}(z)=\left(a_{j j^{\prime}}(z)\right)$ is the $2 \times 2$ scattering matrix. In terms of the modified eigenfunctions:

$$
\mu_{n}^{(1)}(z, t)=\mu_{n}^{(2)}(z, t) \hat{Z}^{n} \mathrm{e}^{-i \omega(z) t \hat{\sigma}_{3}} \mathrm{~A}(z) .
$$

Or, in component form,

$$
\begin{aligned}
& \mu_{n}^{(1, L)}(z, t)=a_{11}(z) \mu_{n}^{(2, L)}(z, t)+z^{-2 n} \mathrm{e}^{2 i \omega(z) t} a_{21}(z) \mu_{n}^{(2, R)}(z, t), \\
& \mu_{n}^{(1, R)}(z, t)=z^{2 n} \mathrm{e}^{-2 i \omega(z) t} a_{12}(z) \mu_{n}^{(2, L)}(z, t)+a_{22}(z) \mu_{n}^{(2, R)}(z, t) .
\end{aligned}
$$

The above relations imply $\mathrm{A}(z)=\lim _{n \rightarrow \infty} \hat{\mathrm{Z}}^{-n} \mathrm{e}^{i \omega(z) t \hat{\sigma}_{3}} \mu_{n}^{(1)}(z, t)=\lim _{n \rightarrow \infty} \Psi_{n}^{(1)}(z, t)$, that is,

$$
\mathrm{A}(z)=\mathrm{I}+\mathrm{Z}^{-1} \sum_{n=-\infty}^{\infty} \hat{\mathrm{Z}}^{-n} \mathrm{e}^{-i \omega(z) t \hat{\sigma}_{3}}\left(\mathrm{Q}_{n}(t) \mu_{n}^{(1)}(z, t)\right) .
$$


The scattering matrix $A(z)$ is independent of time, since $A(z)=\lim _{n \rightarrow \infty} \Psi_{n}^{(1)}(z, t)$, and $\lim _{n \rightarrow \infty} \dot{\Psi}_{n}(z, t)=0$. Equation (3.7) also implies $\operatorname{det} \mathrm{A}(z)=\operatorname{det} \Phi_{\infty}^{(1)}(z, t)=C_{-\infty}$, as well as

$$
\mathrm{A}(z)=C_{n}\left(\begin{array}{cc}
\operatorname{Wr}\left(\Phi_{n}^{(1, L)}, \Phi_{n}^{(2, R)}\right) & \operatorname{Wr}\left(\Phi_{n}^{(1, R)}, \Phi_{n}^{(2, R)}\right) \\
-\operatorname{Wr}\left(\Phi_{n}^{(1, L)}, \Phi_{n}^{(2, L)}\right) & -\operatorname{Wr}\left(\Phi_{n}^{(1, R)}, \Phi_{n}^{(2, L)}\right)
\end{array}\right) .
$$

The analyticity of the eigenfunctions then implies that $a_{11}(z)$ and $a_{22}(z)$ can be analytically continued off the unit circle, respectively into the domains $|z|>1$ and $|z|<1$, but $a_{12}(z)$ and $a_{21}(z)$ cannot. It is also useful to introduce the reflection coefficients

$$
\rho_{1}(z)=a_{21}(z) / a_{11}(z), \quad \rho_{2}(z)=a_{12}(z) / a_{22}(z) .
$$

Symmetries. When $p_{n}(t)=v q_{n}^{*}(t)$, the scattering problem (A.5a) admits an important involution, which can be conveniently written introducing the matrix $\sigma_{v}$ defined in (A.11a). Indeed, when $p_{n}=v q_{n}^{*}$, if $\Phi_{n}(z, t)$ is a solution of (A.5a), so is the matrix

$$
\Phi_{n}^{\prime}(z, t)=\sigma_{v} \Phi_{n}^{*}\left(1 / z^{*}, t\right),
$$

Then, comparing the asymptotic behavior of the first and second columns of the Jost eigenfunctions as $n \rightarrow \pm \infty$ one obtains, for $j=1,2$,

$$
\Phi_{n}^{(j, L)}(z, t)=\sigma_{v}\left(\Phi_{n}^{(j, R)}\left(1 / z^{*}, t\right)\right)^{*}, \quad \Phi_{n}^{(j, R)}(z, t)=v \sigma_{v}\left(\Phi_{n}^{(j, L)}\left(1 / z^{*}, t\right)\right)^{*} .
$$

The above relations imply the following symmetries for the elements of the scattering matrix:

$$
a_{22}(z)=a_{11}^{*}\left(1 / z^{*}\right), \quad a_{21}(z)=v a_{12}^{*}\left(1 / z^{*}\right) .
$$

In turn, these imply $\rho_{2}(z)=v \rho_{1}^{*}\left(1 / z^{*}\right)$.

Discrete spectrum. The proper eigenvalues of the scattering problem (A.5a) are the values $z=z_{j}$ with $\left|z_{j}\right|<1$ and $z=\bar{z}_{j}$ with $\left|\bar{z}_{j}\right|>1$ for which there exist eigenfunctions bounded $\forall n \in \mathbb{Z}$. From the asymptotic behavior of the Jost solutions one can see that such eigenvalues occur whenever the appropriate left- and right-sided Jost solutions are proportional, namely $\Phi_{n}^{(1, L)}\left(\bar{z}_{j}, t\right)=\bar{b}_{j}^{(o)} \Phi_{n}^{(2, R)}\left(\bar{z}_{j}, t\right)$ and $\Phi_{n}^{(1, R)}\left(z_{j}, t\right)=b_{j}^{(o)} \Phi_{n}^{(2, L)}\left(z_{j}, t\right)$, or equivalently:

$\mu_{n}^{(1, L)}\left(\bar{z}_{j}, t\right)=\bar{b}_{j}^{(o)} \bar{z}_{j}^{-2 n} \mathrm{e}^{2 i \omega\left(\bar{z}_{j}\right) t} \mu_{n}^{(2, R)}\left(\bar{z}_{j}, t\right), \quad \mu_{n}^{(1, R)}\left(z_{j}, t\right)=b_{j}^{(o)} z_{j}^{2 n} \mathrm{e}^{-2 i \omega\left(z_{j}\right) t} \mu_{n}^{(2, L)}\left(z_{j}, t\right)$.

The Wronskian representations (3.10) then imply that such eigenvalues are the zeros of the scattering coefficients: $a_{11}\left(\bar{z}_{j}\right)=0$ and $a_{22}\left(z_{j}\right)=0$, respectively. (As in Ref. [6] we assume that $a_{j j}(z) \neq 0$ for all $|z|=1$.) Since no accumulation points of such zeros can exist (because of the sectional analyticity of the scattering coefficients), it follows that there is a finite number of them. As in Ref. [6] we assume all of these zeros are simple. (The case of multiple zeros can be studied as the coalescence of simple zeros, by analogy with the continuum case [44].) Since $a_{j j}(z)$ are even functions [6], $z=z_{j}$ is a zero of $a_{22}(z)$ iff $z=-z_{j}$ is, and similarly for $a_{11}(z)$. Moreover, the symmetries (3.14) imply that $z=z_{j}$ is a zero of $a_{22}(z)$ iff $\bar{z}_{j}=1 / z_{j}^{*}$ is a zero of $a_{11}(z)$. Thus, discrete eigenvalues appear in quartets.

The inverse problem will involve the modified eigenfunctions $\mu_{n}^{(1, L)}(z, t) / a_{11}(z)$ and $\mu_{n}^{(1, R)}(z, t) / a_{22}(z)$. Equations (3.15) imply

$\operatorname{Res}_{z=\bar{z}_{j}}\left[\frac{\mu_{n}^{(1, L)}(z, t)}{a_{11}(z)}\right]=\bar{b}_{j} \bar{z}_{j}^{-2 n} \mathrm{e}^{2 i \omega\left(\bar{z}_{j}\right) t} \mu_{n}^{(2, R)}\left(\bar{z}_{j}, t\right), \quad \operatorname{Res}_{z=z_{j}}\left[\frac{\mu_{n}^{(1, R)}(z, t)}{a_{22}(z)}\right]=b_{j} z_{j}^{2 n} \mathrm{e}^{-2 i \omega\left(z_{j}\right) t} \mu_{n}^{(2, L)}\left(z_{j}, t\right)$,

where $b_{j}=b_{j}^{(o)} / a_{22}^{\prime}\left(z_{j}\right)$ and $\bar{b}_{j}=\bar{b}_{j}^{(o)} / a_{11}^{\prime}\left(\bar{z}_{j}\right)$ are referred to as the norming constants. The symmetries of the scattering problem imply $\bar{b}_{j}=-v\left(b_{j} / z_{j}^{2}\right)^{*}$. 
Asymptotics. The asymptotic behavior of the eigenfunctions as $z \rightarrow 0$ or $z \rightarrow \infty$ can be obtained from (3.5). For example, for $\mu_{n}^{(1)}(z, t)$ it is

$$
\mu_{n}^{(1)}(z, t)=\mathrm{I}+\mathrm{Q}_{n-1} \mathrm{Z}^{-1}+O\left(\mathrm{Z}^{-2}\right) \quad \text { as } \mathrm{z} \rightarrow(\infty, 0)
$$

where $z \rightarrow\left(z_{L}, z_{R}\right)$ indicates $z \rightarrow z_{L}$ in the first column and $z \rightarrow z_{R}$ in the second one, and the asymptotics corresponding to $O\left(Z^{m}\right)$ is defined in Appendix A. Equation (3.17) will allow us to reconstruct the potentials from the asymptotic behavior of $\mu_{n}^{(1)}$ :

$$
\mathrm{Q}_{n}(t)=\lim _{z \rightarrow(\infty, 0)}\left(\mu_{n+1}^{(1)}(z, t)-1\right) Z \text {. }
$$

The asymptotic behavior of $\mu_{n}^{(2)}(z, t)$ is obtained in a slightly different way as that of $\mu_{n}^{(1)}(z, t)$, and the result is also different. More precisely, in Appendix $\mathrm{D}$ we show that

$$
C_{n} \mu_{n}^{(2)}(z, t)=\mathrm{I}-\mathrm{Q}_{n} \mathrm{Z}+O\left(\mathrm{Z}^{2}\right) \quad \text { as } z \rightarrow(0, \infty) .
$$

Also, inserting (3.17) into the diagonal elements of (3.9) one obtains the asymptotic behavior of the analytic scattering coefficients:

$$
a_{11}(z)=1+\frac{1}{z^{2}} \sum_{n=-\infty}^{\infty} q_{n}(t) p_{n}(t)+O\left(1 / z^{4}\right), \quad \text { as } z \rightarrow \infty .
$$

which by symmetry also determines the behavior of $a_{22}(z)$ as $z \rightarrow 0$.

Inverse problem. The inverse problem is the RHP defined by (3.8) for $|z|=1$ :

$$
\begin{aligned}
& \frac{\mu_{n}^{(1, L)}(z, t)}{a_{11}(z)}-\mu_{n}^{(2, L)}(z, t)=z^{-2 n} \mathrm{e}^{2 i \omega(z) t} \rho_{1}(z, t) \mu_{n}^{(2, R)}(z, t), \\
& \frac{\mu_{n}^{(1, R)}(z, t)}{a_{22}(z)}-\mu_{n}^{(2, R)}(z, t)=z^{2 n} \mathrm{e}^{-2 i \omega(z) t} \rho_{2}(z, t) \mu_{n}^{(2, L)}(z, t),
\end{aligned}
$$

where $\rho_{1}(z)$ and $\rho_{2}(z)$ as in (3.11). Unlike the continuum case, the asymptotics of $\mu_{n}^{(2, L)}(z, t)$ as $z \rightarrow \infty$ depends on the values of the potentials $q_{m}(t)$ and $p_{m}(t)$ for all $m \geq n$ through $C_{n}$ [cf. (3.6)]. This problem can be circumvented by introducing the following renormalizations:

$$
\begin{aligned}
& \mathrm{M}_{n}^{-}(z, t)=\left(\begin{array}{cc}
1 & 0 \\
0 & C_{n}
\end{array}\right)\left(\frac{\mu_{n}^{(1, L)}(z, t)}{a_{11}(z)}, \mu_{n}^{(2, R)}(z, t)\right), \\
& \mathrm{M}_{n}^{+}(z, t)=\left(\begin{array}{cc}
1 & 0 \\
0 & C_{n}
\end{array}\right)\left(\mu_{n}^{(2, L)}(z, t), \frac{\mu_{n}^{(1, R)}(z, t)}{a_{22}(z)}\right) .
\end{aligned}
$$

The matrices $\mathrm{M}_{n}^{ \pm}(z, t)$ are sectionally meromorphic for $|z|<1$ and $|z|>1$, respectively. Moreover, (3.19) yields the following jump condition for the matrices $\mathrm{M}_{n}^{ \pm}(z, t)$ on $|z|=1$ :

$$
\mathrm{M}_{n}^{-}(z, t)=\mathrm{M}_{n}^{+}(z, t)\left(\mathrm{I}-\mathrm{J}_{n}(z, t)\right),
$$

where the jump matrix $\mathrm{J}_{n}(z, t)$ is

$$
\mathrm{J}_{n}(z, t)=\left(\begin{array}{cc}
\rho_{1}(z) \rho_{2}(z) & z^{2 n} \mathrm{e}^{-2 i \omega(z) t} \rho_{2}(z) \\
-z^{-2 n} \mathrm{e}^{2 i \omega(z) t} \rho_{1}(z) & 0
\end{array}\right) .
$$

Moreover, $\mathrm{M}_{n}^{ \pm}(z, t)$ have the following asymptotic behavior:

$$
\begin{aligned}
& \mathrm{M}_{n}^{-}(z, t)=\mathrm{I}+\frac{1}{z}\left(\begin{array}{cc}
0 & -q_{n} / C_{n} \\
p_{n-1} C_{n} & 0
\end{array}\right)+O\left(1 / z^{2}\right) \quad \text { as } z \rightarrow \infty, \\
& \mathrm{M}_{n}^{+}(z, t)=\left(\begin{array}{cc}
1 / C_{n} & 0 \\
0 & C_{n}
\end{array}\right)+z\left(\begin{array}{cc}
0 & q_{n-1} \\
-p_{n} & 0
\end{array}\right)+O\left(z^{2}\right) \quad \text { as } z \rightarrow 0 .
\end{aligned}
$$

In the absence of a discrete spectrum [that is, if $a_{11}(z, t) \neq 0$ for $|z|>1$ and $a_{22} \neq 0$ for $|z|<1$ ] the matrix functions $\mathrm{M}_{n}^{ \pm}(x, t, k)$ are analytic in their respective domains. 
In particular, (3.21a) allows the RHP (3.20) to be solved via the Cauchy projectors $P^{ \pm}$ over the unit circle, as in the linear case. Of course, unlike the linear case the solution is now expressed in terms of a matrix integral equation:

$$
\mathrm{M}_{n}^{+}(z, t)=\mathrm{I}+\frac{1}{2 \pi i} \int_{|\zeta|=1} \mathrm{M}_{n}^{+}(\zeta, t) \frac{\mathrm{J}_{n}(\zeta, t)}{\zeta-z} \mathrm{~d} \zeta
$$

The asymptotic behavior of $\mathrm{M}_{n}^{+}(z, t)$ as $z \rightarrow 0$ is easily obtained from (3.22):

$$
\mathrm{M}_{n}^{+}(z, t)=\mathrm{I}+\frac{1}{2 \pi i} \int_{|\zeta|=1} \mathrm{M}_{n}^{+}(\zeta, t) \mathrm{J}_{n}(\zeta, t) \frac{\mathrm{d} \zeta}{\zeta}+\frac{z}{2 \pi i} \int_{|\zeta|=1} \mathrm{M}_{n}^{+}(\zeta, t) \mathrm{J}_{n}(\zeta, t) \frac{\mathrm{d} \zeta}{\zeta^{2}}+O\left(z^{2}\right)
$$

Comparing the limit as $z \rightarrow 0$ of (3.23) with (3.21b), we see that the off-diagonal portion of the first integral in (3.23) is zero, a fact which is not entirely obvious otherwise. (This integral is missing in the corresponding formula in Ref. [6].) Then, comparing the $(1,2)$ components of (3.23) and (3.21b) we obtain the reconstruction formula for the solution of the IVP:

$$
q_{n}(t)=\frac{1}{2 \pi i} \int_{|z|=1} z^{2 n} \mathrm{e}^{-2 i \omega(z) t} \rho_{2}(z)\left(\mu_{n+1}^{(2)}(z, t)\right)_{11} \mathrm{~d} z
$$

Linear limit. As in the continuum limit, the IST is the nonlinear analogue of the linear transform pair. Namely, if $\mathrm{Q}_{n}=O(\varepsilon)$, then $\mu_{n}^{(1)}=\mathrm{I}+O(\varepsilon)$ and

$$
\mathrm{A}(z)=\mathrm{I}+\mathrm{Z}^{-1} \sum_{n=-\infty}^{\infty} \hat{\mathrm{Z}}^{-n} \mathrm{e}^{-i \omega(z) t \hat{\sigma}_{3}} \mathrm{Q}_{n}(t)+O\left(\varepsilon^{2}\right)
$$

Thus

$$
\rho_{2}(\zeta)=\sum_{n=-\infty}^{\infty} \zeta^{-2 n-1} \mathrm{e}^{-2 i \omega(\zeta) t} q_{n}(t)+O\left(\varepsilon^{2}\right)=\frac{1}{\zeta} \hat{q}\left(\zeta^{2}, 0\right)+O\left(\varepsilon^{2}\right)
$$

where $\hat{q}(z, t)$ is the linear $z$-transform defined in (2.1). Similarly,

$q_{n}(t)=\frac{1}{2 \pi i} \int_{|\zeta|=1} \zeta^{2 n} \mathrm{e}^{-2 i \omega(\zeta) t} \rho_{2}(\zeta) \mathrm{d} \zeta+O\left(\varepsilon^{2}\right)=\frac{1}{2 \pi i} \int_{|z|=1} z^{n-1} \mathrm{e}^{-i \omega_{\mathrm{dls}}(z) t} \hat{q}(z, 0) \mathrm{d} z+O\left(\varepsilon^{2}\right)$,

where the change of variable $\zeta^{2}=z$ was performed in the RHS of (3.24), and where $\omega_{\mathrm{idnls}}(\zeta)=\frac{1}{2} \omega_{\mathrm{dls}}\left(\zeta^{2}\right)$, as discussed in section 2.1 .

\subsection{The Ablowitz-Ladik system on the naturals}

We now consider the IBVP for the IDNLS. That is, we solve (1.2) with $n \in \mathbb{N}, t \in \mathbb{R}^{+}$and with $q_{n}(0)$ and $q_{0}(t)$ given. The approach we will follow is a combination of the method for the IVP for the IDNLS on the integers and that for the IBVP for the DLS on the naturals.

Eigenfunctions and analyticity. Making use of the modified eigenfunction $\Psi_{n}(z, t)$ in $(3.3)$, we define three eigenfunctions $\mu_{n}^{(j)}(z, t)$ which reduce to the identity matrix respectively when $(n, t)=(0,0)$, as $(n, t) \rightarrow(\infty, t)$ and at $(n, t)=(0, T)$ :

$$
\begin{aligned}
& \mu_{n}^{(1)}(z, t)=\mathrm{I}+\mathrm{Z}^{-1} \sum_{m=0}^{n-1} \hat{Z}^{n-m}\left(\mathrm{Q}_{m}(t) \mu_{m}^{(1)}(z, t)\right)+\hat{Z}^{n} \int_{0}^{t} \mathrm{e}^{-i \omega(z)\left(t-t^{\prime}\right) \hat{\sigma}_{3}}\left(\mathrm{H}_{0}\left(z, t^{\prime}\right) \mu_{0}^{(1)}\left(z, t^{\prime}\right)\right) \mathrm{d} t^{\prime} \\
& \mu_{n}^{(2)}(z, t)=\mathrm{I}-\mathrm{Z}^{-1} \sum_{m=n}^{\infty} \hat{Z}^{n-m}\left(\mathrm{Q}_{m}(t) \mu_{m}^{(2)}(z, t)\right), \\
& \mu_{n}^{(3)}(z, t)=\mathrm{I}+\mathrm{Z}^{-1} \sum_{m=0}^{n-1} \hat{Z}^{n-m}\left(\mathrm{Q}_{m}(t) \mu_{m}^{(3)}(z, t)\right)-\hat{Z}^{n} \int_{t}^{T} \mathrm{e}^{-i \omega(z)\left(t-t^{\prime}\right) \hat{\sigma}_{3}}\left(\mathrm{H}_{0}\left(z, t^{\prime}\right) \mu_{0}^{(3)}\left(z, t^{\prime}\right)\right) \mathrm{d} t^{\prime}
\end{aligned}
$$



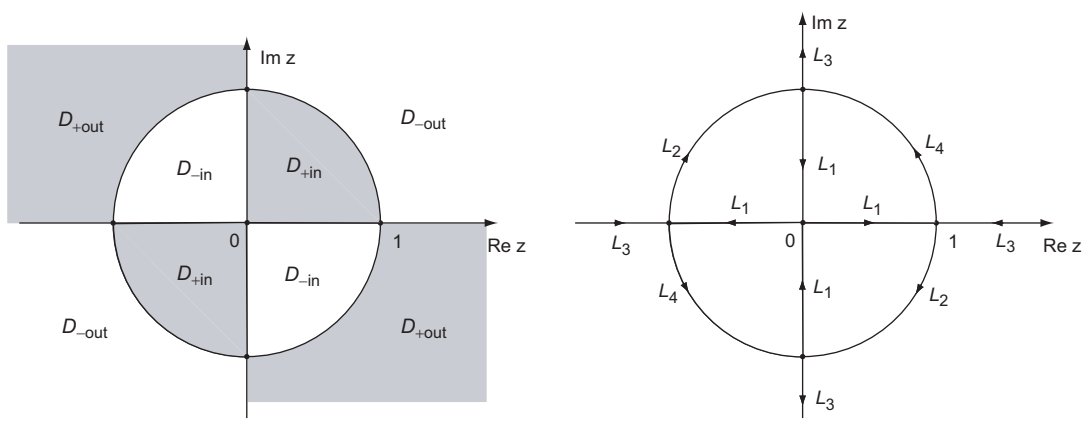

Figure 3. (Left) The regions $D_{+}$(shaded) and $D_{-}$(white) of the $z$-plane where $\operatorname{Im}[\omega(z)] \gtrless 0$ in the nonlinear case, with $D_{ \pm}=D_{ \pm \text {in }} \cup D_{ \pm \text {out }}$. (Right) The contours $L_{1}, \ldots, L_{4}$ that define the Riemann-Hilbert problem (see text for details)

Note that $\mu_{n}^{(2)}(z, t)$ coincides with the eigenfunction in the IVP, defined in (3.5). As in the linear case, we partition the complex $z$-plane into the domains $D_{ \pm}$defined as $D_{ \pm}=\{z \in \mathbb{C}$ : $\operatorname{Im} \omega(z) \gtrless 0\}$. We then write $D_{ \pm}=D_{ \pm \text {in }} \cup D_{ \pm \text {out }}$ where the subscripts "in" and "out" denote the portions of $D_{ \pm}$inside and outside the unit disk, respectively. That is (cf. Fig 3),

$$
\begin{aligned}
& D_{\text {+in }}=\{z \in \mathbb{C}:|z|<1 \wedge \arg z \in(0, \pi / 2) \cup(\pi, 3 \pi / 2)\}, \\
& D_{- \text {in }}=\{z \in \mathbb{C}:|z|<1 \wedge \arg z \in(\pi / 2, \pi) \cup(3 \pi / 2,2 \pi)\}, \\
& D_{\text {+out }}=\{z \in \mathbb{C}:|z|>1 \wedge \arg z \in(\pi / 2, \pi) \cup(3 \pi / 2,2 \pi)\}, \\
& D_{\text {-out }}=\{z \in \mathbb{C}:|z|>1 \wedge \arg z \in(0, \pi / 2) \cup(\pi, 3 \pi / 2)\} .
\end{aligned}
$$

Then, in a similar way as in the IVP on the whole line and the IBVP in the linear problem, we can obtain the regions of analyticity and boundedness of the eigenfunctions. More precisely, writing again $\mu_{n}^{(j)}(z, t)=\left(\mu_{n}^{(j, L)}, \mu_{n}^{(j, R)}\right)$, we have:

- $\mu_{n}^{(1)}(z, t)$ and $\mu_{n}^{(3)}(z, t)$ are analytic in the punctured complex $z$-plane $\mathbb{C}^{[\emptyset]}$;

- $\mu_{n}^{(1, L)}(z, t)$ is continuous and bounded in $\bar{D}_{\text {+out }}$;

- the restriction of $\mu_{n}^{(1, R)}(z, t)$ to $D_{- \text {in }}$ is continuous and bounded in $\bar{D}_{- \text {in }}$;

- $\mu_{n}^{(3, L)}(z, t)$ is continuous and bounded in $\bar{D}_{- \text {out }}$;

- the restriction of $\mu_{n}^{(3, R)}(z, t)$ to $D_{+ \text {in }}$ is continuous and bounded in $\bar{D}_{+ \text {in }}$;

- $\mu_{n}^{(2, L)}(z, t)$ is analytic for $|z|<1$ and continuous and bounded for $|z| \leq 1$;

- $\mu_{n}^{(2, R)}(z, t)$ is analytic for $|z|>1$ and continuous and bounded for $|z| \geq 1$.

The analyticity of the eigenfunctions is formally proven via Neumann series as in the IVP [6] and as in the IVP for the continuum case [29]. However, showing the continuity of $\mu_{n}^{(1, R)}(z, t)$ and $\mu_{n}^{(3, R)}(z, t)$ at $z=0$ is nontrivial, and it requires studying the asymptotic behavior of the eigenfunctions as $z \rightarrow 0$ (see Appendix D).

Scattering matrices. The relation $\operatorname{det} \Phi_{n+1}=\left(1-q_{n} p_{n}\right) \operatorname{det} \Phi_{n}$ still holds. Therefore $\operatorname{det} \Phi_{n}^{(1)}$ and $\operatorname{det} \Phi_{n}^{(2)}$ are still given by (3.6), and $\operatorname{det} \Phi_{n}^{(1)}=\operatorname{det} \Phi_{n}^{(3)}$. [Note that $\mu_{t}=\mathrm{L} \mu+\mu \mathrm{R}$ implies $(\operatorname{det} \mu)_{t}=\operatorname{tr}(\mathrm{L}+\mathrm{R}) \operatorname{det} \mu$, and in our case both $\mathrm{L}$ and $\mathrm{R}$ are traceless; cf. (A.5) and Appendix A.] Hence, under the same regularity hypotheses as before, $\Phi_{n}^{(1)}, \Phi_{n}^{(2)}$ and $\Phi_{n}^{(3)}$ 
are each fundamental solutions of the Lax pair (A.5). We can therefore write the following relations among the modified eigenfunctions:

$$
\begin{aligned}
& \mu_{n}^{(2)}(z, t)=\mu_{n}^{(1)}(z, t) \hat{Z}^{n} \mathrm{e}^{-i \omega(z) t \hat{\sigma}_{3}} \mathrm{~S}(z), \\
& \mu_{n}^{(3)}(z, t)=\mu_{n}^{(1)}(z, t) \hat{Z}^{n} \mathrm{e}^{-i \omega(z) t \hat{\sigma}_{3}} \mathrm{~S}(z, T),
\end{aligned}
$$

which hold wherever all terms are defined, namely: the first column of (3.27a) holds for $0<|z| \leq 1$, the second column for $|z| \geq 1$ and (3.27b) holds $\forall z \neq 0$. Thus

$$
\mathrm{s}(z)=\mu_{0}^{(2)}(z, 0), \quad \mathrm{S}(z, T)=\left(\mathrm{e}^{i \omega(z) T \hat{\sigma}_{3}} \mu_{0}^{(1)}(z, T)\right)^{-1} .
$$

Equation (3.28) allows us to write integral representations for the scattering matrices:

$$
\begin{aligned}
& \mathrm{S}(z)=\mathrm{I}-\mathrm{Z}^{-1} \sum_{n=0}^{\infty} \hat{Z}^{-n}\left(\mathrm{Q}_{n}(t) \mu_{n}^{(2)}(z, 0)\right), \\
& \mathrm{S}^{-1}(z, T)=\mathrm{I}+\int_{0}^{T} \mathrm{e}^{i \omega(z) t \hat{\sigma}_{3}}\left(\mathrm{H}_{0}(z, t) \mu_{0}^{(1)}(z, t)\right) \mathrm{d} t .
\end{aligned}
$$

Note that $\mathrm{s}(z)$ is again independent of time, since $\mathrm{s}^{-1}(z)=\lim _{n \rightarrow \infty} \hat{\mathrm{Z}}^{-n} \mathrm{e}^{i \omega(z) t \hat{\sigma}_{3}} \mu_{n}^{(1)}(z, t)=$ $\lim _{n \rightarrow \infty} \Psi_{n}^{(1)}(z, t)$, as in the IVP. Note also that (3.27) implies

$$
\operatorname{det} \mathrm{s}(z)=1 / C_{0}, \quad \operatorname{det} \mathrm{S}(z, T)=1 .
$$

The analyticity properties of $\mu_{0}^{(2)}(z, t)$ are the same as those of $\mu_{n}^{(2)}(z, t)$. However, $\mu_{0}^{(1)}(z, t)$ enjoys larger domains of analyticity and boundedness than $\mu_{n}^{(1)}(z, t)$. The analyticity and boundedness regions of the scattering matrices are determined correspondingly via (3.28):

- $\mathrm{s}_{L}(z)$ is analytic for $|z|<1$ and continuous and bounded for $|z| \leq 1$; while $\mathrm{s}_{R}(z)$ is analytic in $|z|>1$ and continuous and bounded for $|z| \geq 1$;

- $\mathrm{S}(z, T)$ is analytic in $\mathbb{C}^{[\emptyset]}$; moreover, $\mathrm{S}_{L}(z, T)$ is continuous and bounded in $\bar{D}_{-}$, while $\mathrm{S}_{R}(z, T)$ is continuous and bounded in $\bar{D}_{+}$.

The above boundedness properties of $\mathrm{S}(z, T)$ can be obtained as follows. Let us write the matrix $\mathrm{S}(z, T)$ as

$$
\mathrm{S}(z, T)=\left(\begin{array}{ll}
A(z, T) & \tilde{B}(z, T) \\
B(z, T) & \tilde{A}(z, T)
\end{array}\right) .
$$

As we show below, the symmetries of the problem imply that $\tilde{A}(z, T)$ and $\tilde{B}(z, T)$ can be obtained respectively in terms of $A(z, T)$ and $B(z, T)$. Hence, we only need to discuss the properties of $A(z, T)$ and $B(z, T)$. Recall that $\mathrm{S}(z, T)$ is an entire function of $z$, and note that (3.30) implies

$$
\mathrm{S}^{-1}(z, T)=\left(\begin{array}{cc}
\tilde{A}(z, T) & -\tilde{B}(z, T) \\
-B(z, T) & A(z, T)
\end{array}\right) .
$$

Then (3.28) and the analyticity properties of $\mu_{0}^{(1)}(z, T)$ imply that $A(z, T)$ is bounded in $\bar{D}_{-}$. Also, (3.29b) and the integral representation (3.25a) with $n=0$ can be used to write a Neumann series for $\mathrm{S}^{-1}(z, T)$, which in turn can be used to prove analyticity and boundedness of $B(z, T)$ in $\bar{D}_{-}$.

The involution symmetry discussed when dealing with the IVP is a local property. Therefore, when $p_{n}(t)=v q_{n}^{*}(t)$, it also applies for the IBVP. That is, (3.13) still holds, as does (5.30) for $j=1,2,3$. This implies

$$
\mathrm{s}(z)=\left(\begin{array}{cc}
a(z) & v b^{*}\left(1 / z^{*}\right) \\
b(z) & a^{*}\left(1 / z^{*}\right)
\end{array}\right), \quad \mathrm{S}(z, T)=\left(\begin{array}{cc}
A(z, T) & v B^{*}\left(1 / z^{*}, T\right) \\
B(z, T) & A^{*}\left(1 / z^{*}, T\right)
\end{array}\right) .
$$

Note that (3.30) imply

$$
\begin{aligned}
& a(z) a^{*}\left(1 / z^{*}\right)-v b(z) b^{*}\left(1 / z^{*}\right)=1 / C_{0}, \\
& A(z, T) A^{*}\left(1 / z^{*}, T\right)-v B(z, T) B^{*}\left(1 / z^{*}, T\right)=1 .
\end{aligned}
$$


Asymptotics. Since $\mu_{n}^{(2)}(z, t)$ coincides with (3.5), its asymptotics as $z \rightarrow(0, \infty)$ is still given by (3.18). Also, in Appendix D we show that, even though the definition of $\mu_{n}^{(1)}(z, t)$ and $\mu_{n}^{(3)}(z, t)$ involves time integrals, it is still $\mu_{n}^{(j)}(z, t)=\mathrm{I}+O\left(\mathrm{Z}^{-1}\right)$ as $z \rightarrow(\infty, 0)$ for $j=1$ and $j=3$ in their respective domains of boundedness. More precisely, for all $n>0$ it is

$$
\mu_{n}^{(j)}(z, t)=\mathrm{I}+\mathrm{Q}_{n-1}(t) \mathrm{Z}^{-1}+O\left(\mathrm{Z}^{-2}\right), \quad \text { as } z \rightarrow(\infty, 0)
$$

for $j=1,3$, and the limits are restricted the appropriate regions of the complex plane, where the corresponding columns are bounded. For $n=0$ it is instead

$$
\begin{aligned}
& \mu_{0}^{(1)}(z, t)=\mathrm{I}+\left(\mathrm{Q}_{-1}(t)-\mathrm{e}^{-i \omega(z) t \hat{\sigma}_{3}} \mathrm{Q}_{-1}(0)\right) \mathrm{Z}^{-1}+O\left(\mathrm{Z}^{-2}\right), \\
& \mu_{0}^{(3)}(z, t)=\mathrm{I}+\left(\mathrm{Q}_{-1}(t)-\mathrm{e}^{-i \omega(z)(t-T) \hat{\sigma}_{3}} \mathrm{Q}_{-1}(T)\right) \mathrm{Z}^{-1}+O\left(\mathrm{Z}^{-2}\right),
\end{aligned}
$$

as $z \rightarrow(\infty, 0)$. The above yield, for all $n \geq 0$,

$$
\mathrm{Q}_{n-1}(t)=\lim _{z \rightarrow(\infty, 0)}\left(\mu_{n}^{(j)}(z, t)-1\right) Z, \quad \text { for } j=1,3 .
$$

Also, the asymptotic behavior of the eigenfunctions determines that of the scattering matrices. In particular, from the second of (3.28) we have

$$
A^{*}\left(1 / z^{*}, T\right)=1+O\left(1 / z^{2}\right), B^{*}\left(1 / z^{*}, T\right)=O(1 / z) \text { as } z \rightarrow \infty \text { in } \bar{D}_{+ \text {out }},(3.34 a)
$$

while $(3.27 b)$ implies

$$
A^{*}\left(1 / z^{*}, T\right)=1+O\left(z^{2}\right), \quad B^{*}\left(1 / z^{*}, T\right)=O(z) \quad \text { as } z \rightarrow 0 \text { in } \bar{D}_{+ \text {in }} .
$$

Similarly, (3.18) and (3.27a) yield

$$
a^{*}\left(1 / z^{*}\right)=1 / C_{0}+O\left(1 / z^{2}\right), \quad b^{*}\left(1 / z^{*}\right)=O(1 / z) \quad \text { as } z \rightarrow \infty \text { in } \bar{D}_{+ \text {out }} .
$$

Riemann-Hilbert problem, solution and reconstruction formula. We now formulate the RHP whose solution will enable us to obtain a representation for the solution of the AL system on the naturals. For later reference, we introduce the quantities

with

$$
\gamma(z)=\frac{v b^{*}(z)}{a(z)}, \quad R(z, t)=\frac{B^{*}\left(1 / z^{*}, t\right)}{A^{*}\left(1 / z^{*}, t\right)}, \quad \Gamma(z)=\frac{B(z, T)}{a^{*}\left(1 / z^{*}\right) d^{*}\left(1 / z^{*}\right)},
$$

$$
d(z)=a(z) A^{*}\left(1 / z^{*}, T\right)-v b(z) B^{*}\left(1 / z^{*}, T\right) .
$$

Note that $R(z, T)$ is defined $\forall z \in \mathbb{C}$ except where $A^{*}\left(1 / z^{*}, T\right)=0, \Gamma(z)$ is defined for $z \in L_{3} \cup L_{4}$, $d(z)$ for $z \in \bar{D}_{ \pm \text {in }}$, and $\gamma(z)$ for $|z|=1$. Moreover, $d^{*}\left(1 / z^{*}\right)=1 / C_{0}+O\left(1 / z^{2}\right)$ as $z \rightarrow \infty$. In the analysis of linearizable BCs, it will be useful to write $\Gamma^{*}\left(1 / z^{*}\right)$ in terms of only $a(z), b(z)$ and $R(z, T)$ as

$$
\Gamma^{*}\left(1 / z^{*}\right)=\frac{R(z, T)}{a(z)(a(z)-v b(z) R(z, T))} .
$$

Finally, we introduce the normalization matrix $\mathrm{C}_{n}=\operatorname{diag}\left(1 / C_{0}, C_{n}\right)$.

We are now ready to formulate the RHP, which we do using (3.27). We introduce the matrix functions $\mathrm{M}_{n}^{ \pm}(z, t)$ defined as:

$$
\begin{aligned}
& \mathrm{M}_{n}^{+}(z, t)= \begin{cases}\mathrm{C}_{n}\left(\mu_{n}^{(2, L)}(z, t), \frac{\mu_{n}^{(3, R)}(z, t)}{d(z)}\right), & z \in D_{+\mathrm{in}}, \\
\mathrm{C}_{n}\left(\frac{\mu_{n}^{(1, L)}(z, t)}{a^{*}\left(1 / z^{*}\right)}, \mu_{n}^{(2, R)}(z, t)\right), & z \in D_{+ \text {out }},\end{cases} \\
& \mathrm{M}_{n}^{-}(z, t)=\left\{\begin{array}{l}
\mathrm{C}_{n}\left(\mu_{n}^{(2, L)}, \frac{\mu_{n}^{(1, R)}}{a(z)}\right), \quad z \in D_{\text {-in }}, \\
\mathrm{C}_{n}\left(\frac{\mu_{n}^{(3, L)}}{d^{*}\left(1 / z^{*}\right)}, \mu_{n}^{(2, R)}\right), \quad z \in D_{- \text {out }} .
\end{array}\right.
\end{aligned}
$$


Note that $\mathrm{M}_{n}^{ \pm}(z, t)$ are sectionally meromorphic respectively for $z \in D_{+}$and $z \in D_{-}$. Moreover, after some tedious but straightforward algebra, equations (3.27) yield the jump conditions as

$$
\mathrm{M}_{n}^{-}(z, t)=\mathrm{M}_{n}^{+}(z, t)\left(\mathrm{I}-\mathrm{J}_{n}(z, t)\right), \quad z \in L,
$$

where the contours $L=L_{1} \cup L_{2} \cup L_{3} \cup L_{4}$ are (cf. Fig. 3)

$L_{1}=\bar{D}_{\text {+in }} \cap \bar{D}_{\text {-in }}, \quad L_{2}=\bar{D}_{\text {-in }} \cap \bar{D}_{\text {+out }}, \quad L_{3}=\bar{D}_{\text {+out }} \cap \bar{D}_{\text {-out }}, \quad L_{4}=\bar{D}_{\text {+in }} \cap \bar{D}_{\text {-out }}$, and the jump matrices $\mathrm{J}_{n}^{(1)}, \ldots, \mathrm{J}_{n}^{(4)}$ are defined by

$$
\begin{aligned}
& \mathrm{J}_{n}^{(1)}(z, t)=\left(\begin{array}{cc}
0 & v z^{2 n} \mathrm{e}^{-2 i \omega(z) t} \Gamma^{*}\left(1 / z^{*}, T\right) \\
0 & 0
\end{array}\right), \quad z \in L_{1}, \\
& \mathrm{~J}_{n}^{(2)}(z, t)=\left(\begin{array}{cc}
1-1 / C_{0} & z^{2 n} \mathrm{e}^{-2 i \omega(z) t} \gamma(z) \\
-v z^{-2 n} \mathrm{e}^{2 i \omega(z) t} \gamma^{*}(z) & 1-C_{0}\left(1-v|\gamma(z)|^{2}\right)
\end{array}\right), \quad z \in L_{2}, \\
& \mathrm{~J}_{n}^{(3)}(z, t)=\left(\begin{array}{cc}
0 & 0 \\
-z^{-2 n} \mathrm{e}^{2 i \omega(z) t} \Gamma(z, T) & 0
\end{array}\right), \quad z \in L_{3}, \\
& \mathrm{~J}_{n}^{(4)}(z, t)=\mathrm{I}-\left(\mathrm{I}-\mathrm{J}_{n}^{(1)}\right)\left(\mathrm{I}-\mathrm{J}_{n}^{(2)}\right)^{-1}\left(\mathrm{I}-\mathrm{J}_{n}^{(3)}\right), \quad z \in L_{4} .
\end{aligned}
$$

As in the IVP, we first consider the case in which no discrete spectrum is present. For the IBVP, this corresponds to assuming that $a(z) \neq 0$ for $z \in D_{- \text {in }}$ and $d(z) \neq 0$ for $z \in D_{+ \text {in }}$. In this case, the matrix functions $\mathrm{M}_{n}^{ \pm}(z, t)$ are analytic in their respective domains. Also, $\mathrm{M}_{n}(z, t) \rightarrow \mathrm{I}$ as $z \rightarrow \infty$ thanks to (3.18), (3.32a), (3.34) and (3.35). Hence the matrix RHP (3.36) is solved by the Cauchy projectors $P^{ \pm}$over the contour $L$, namely $P^{ \pm}=1 /(2 \pi i) \int_{L}\left[1 /\left(k^{\prime}-k\right)\right] \mathrm{d} k^{\prime}$. That is,

$$
\mathrm{M}_{n}^{+}(z, t)=\mathrm{I}+\frac{1}{2 \pi i} \int_{L} \mathrm{M}_{n}^{+}(\zeta, t) \frac{\mathrm{J}_{n}(\zeta, t)}{\zeta-z} \mathrm{~d} \zeta
$$

Equation (3.37) also yields the asymptotic expansion of $\mathrm{M}_{n}^{+}(z, t)$ as $z \rightarrow 0$, namely,

$$
\mathrm{M}_{n}^{+}(z, t)=\mathrm{I}+\frac{1}{2 \pi i} \int_{L} \mathrm{M}_{n}^{+}(\zeta, t) \mathrm{J}_{n}(\zeta, t) \frac{\mathrm{d} \zeta}{\zeta}+\frac{z}{2 \pi i} \int_{L} \mathrm{M}_{n}^{+}(\zeta, t) \mathrm{J}_{n}(\zeta, t) \frac{\mathrm{d} \zeta}{\zeta^{2}}+O\left(z^{2}\right)
$$

Note that we can write (3.38) as

$$
\mathrm{M}_{n}^{+}(z, t)=\operatorname{diag}\left[1 /\left(C_{0} C_{n}\right), C_{0} C_{n}\right]+\frac{z}{2 \pi i} \int_{L} \mathrm{M}_{n}^{+}(\zeta, t)\left(\mathrm{I}-\mathrm{J}_{n}(\zeta, t)\right) \frac{\mathrm{d} \zeta}{\zeta^{2}}+O\left(z^{2}\right) .
$$

Now note that the matrix $\mathrm{C}_{n}^{-1} \mathrm{M}_{n}(z, t)$ satisfies the $n$-part of the Lax pair (A.5a). Also, thanks to (3.18), (3.32a) and (3.34), it is

$$
\mathrm{C}_{n}^{-1} \mathrm{M}_{n}^{+}(z, t)=\operatorname{diag}\left[1 / C_{n}, C_{0}\right]+O(z) \quad \text { as } z \rightarrow 0 .
$$

Hence, substituting the asymptotic expansion of $\mathrm{M}_{n}(z, t)$ into (A.5a) and comparing the (1,2)components of the $O(z)$ terms, we can recover the scattering potentials as

$$
q_{n}(t)=\lim _{z \rightarrow 0}\left(\mathrm{M}_{n+1}^{+}(z, t)-\mathrm{I}\right)_{12} / z .
$$

Taking the (1,2)-component of (3.39) and comparing with (3.40), we then obtain the reconstruction formula for the solution of the IDNLS equation on the natural numbers:

$$
\begin{gathered}
q_{n}(t)=-\frac{1}{2 \pi i} \int_{|z|=1} z^{2 n} \mathrm{e}^{-2 i \omega(z) t} \gamma(z)\left(\mathrm{M}_{n+1}^{+}(z, t)\right)_{11} \mathrm{~d} z+\frac{v}{2 \pi i} \int_{L_{1}} z^{2 n} \mathrm{e}^{-2 i \omega(z) t} \Gamma^{*}\left(1 / z^{*}\right)\left(\mathrm{M}_{n+1}^{+}(z, t)\right)_{11} \mathrm{~d} z \\
+\frac{1}{2 \pi i} \int_{L_{2}}\left(v C_{0}|\gamma(z)|^{2}-C_{0}+1\right)\left(\mathrm{M}_{n+1}^{+}(z, t)\right)_{12} \frac{\mathrm{d} z}{z^{2}}+\frac{1}{2 \pi i}\left(1-\frac{1}{C_{0}}\right) \int_{L_{4}}\left(\mathrm{M}_{n+1}^{+}(z, t)\right)_{12} \frac{\mathrm{d} z}{z^{2}} \\
+\frac{1}{2 \pi i} \frac{v}{C_{0}} \int_{L_{4}} z^{2 n} \mathrm{e}^{-2 i \omega(z) t} \Gamma^{*}\left(1 / z^{*}\right)\left(\mathrm{M}_{n+1}^{+}(z, t)\right)_{11} \mathrm{~d} z .
\end{gathered}
$$


Global relation. As in the linear problem and the continuum limit, the unknown boundary datum can be obtained in terms of the known initial-boundary conditions using the global relation and the symmetries of the system.

Integrating (3.4) around the boundary of the region $\mathbb{N}_{0} \times[0, t]$, one obtains

$$
\mathrm{Z} \int_{0}^{t} \mathrm{e}^{i \omega(z) t^{\prime} \hat{\sigma}_{3}}\left(\mathrm{H}_{0}\left(z, t^{\prime}\right) \mu_{0}\left(z, t^{\prime}\right)\right) \mathrm{d} t^{\prime}+\mathrm{e}^{i \omega(z) t^{\prime} \hat{\sigma}_{3}} \sum_{n=0}^{\infty} \hat{Z}^{-n}\left(\mathrm{Q}_{n}(t) \mu_{n}(z, t)\right)=\sum_{n=0}^{\infty} \hat{Z}^{-n}\left(\mathrm{Q}_{n}(0) \mu_{n}(z, 0)\right) \text {. }
$$

When (3.42) is evaluated with $\mu_{n}(z, t) \equiv \mu_{n}^{(2)}(z, t)$ and $t=T$, the first and second columns of the resulting equation are valid respectively for $z \in \bar{D}_{ \pm \text {in }}$ and $z \in \bar{D}_{ \pm \text {out }}$. Moreover, the RHS of (3.42) becomes $Z(I-s(z))$ thanks to (3.29a). Finally, using (3.27), we can write the first term and the second term in (3.42) respectively as $Z\left(\mathrm{~S}^{-1}(z, T)-\mathrm{I}\right) \mathrm{s}(z)$ and $\mathrm{Z}\left(\mathrm{I}-\mathrm{e}^{i \omega(z) t \hat{\sigma}_{3}} \mu_{0}^{(2)}(z, T)\right)$. We therefore have the following global relation in terms of the scattering data:

$$
\mathrm{S}^{-1}(z, T) \mathrm{S}(z)=\mathrm{I}-\mathrm{e}^{i \omega(z) T \hat{\sigma}_{3}} \mathrm{G}(z, T),
$$

where

$$
\mathrm{G}(z, t)=\mathrm{Z}^{-1} \sum_{n=0}^{\infty} \hat{\mathrm{Z}}^{-n}\left(\mathrm{Q}_{n} \mu_{n}^{(2)}(z, t)\right) .
$$

Like for (3.42), the first and second column of (3.43) are respectively valid for $|z| \leq 1$ and $|z| \geq 1$. Also, from the analyticity domains of $\mu_{n}^{(2)}(z, t)$ it follows that $\mathrm{G}_{L}(z, t)$ is analytic in $|z|<1$ and $\mathrm{G}_{R}(z, t)$ is analytic in $|z|>1$. Taking the $(1,2)$ component of $(3.43)$ we have

$$
A^{*}\left(1 / z^{*}, T\right) b^{*}\left(1 / z^{*}\right)-B^{*}\left(1 / z^{*}, T\right) a^{*}\left(1 / z^{*}\right)=-v \mathrm{e}^{2 i \omega(z) T} G(z, T),|z|>1,
$$

where

$$
G(z, T)=\sum_{n=0}^{\infty} z^{-2 n-1} q_{n}(T)\left(\mu_{n}^{(2)}(z, T)\right)_{22} .
$$

Also note that the RHS of (3.44) is bounded for $z \in \bar{D}_{+ \text {out }}$. Then, for $z \in \bar{D}_{+ \text {out }}$ the RHS vanishes in the limit $T \rightarrow \infty$, implying

$$
A^{*}\left(1 / z^{*}, T\right) b^{*}\left(1 / z^{*}\right)-B^{*}\left(1 / z^{*}, T\right) a^{*}\left(1 / z^{*}\right)=0, \quad z \in \bar{D}_{\text {+out }} .
$$

For finite values of $T$, letting $r(z)=b(z) / a(z)$, the global relation is now

$$
B^{*}\left(1 / z^{*}, T\right)-r\left(1 / z^{*}\right) A^{*}\left(1 / z^{*}, T\right)=v \mathrm{e}^{2 i \omega(z) T} G(z, T) / a^{*}\left(1 / z^{*}\right) .
$$

Since $G(z, t)=O(1 / z)$ as $z \rightarrow \infty$ for $z \in D_{+ \text {out }}$, multiplying by $z \mathrm{e}^{-2 i \omega(z) t}$ and integrating over $\partial \tilde{D}_{\text {+out }}\left[\right.$ where $\left.\tilde{D}_{\text {+out }}=\left\{D_{\text {+out }} \wedge \operatorname{Im} z>0\right\}\right]$ we obtain the integral relation:

$$
\int_{\partial \tilde{D}_{\text {+out }}} z \mathrm{e}^{-2 i \omega(z) t}\left(B^{*}\left(1 / z^{*}, T\right)-r\left(1 / z^{*}\right) A^{*}\left(1 / z^{*}, T\right)\right) \mathrm{d} z=0 .
$$

This is the discrete analogue of the one that in the continuum case is used to obtain the Dirichlet-to-Neumann map [28]. In the discrete case, however, the unknown boundary datum can be obtained using an alternative, simpler method, as we will show in section 4 .

Linear limit. The linear limit of the solution (3.41) of the IBVP for the IDNLS equation coincides with the solution of the IBVP for the DLS equation, as we show next. Suppose $\mathrm{Q}_{n}(t)=O(\varepsilon)$. From (3.25) it follows that $\mu_{n}=\mathrm{I}+O(\varepsilon)$. Recalling (3.29a), we obtain, to $O(\varepsilon)$ :

$\gamma(z)=-\frac{1}{z} \hat{q}\left(z^{2}, 0\right), \quad d(z)=1, \quad C_{0}=1, \quad \Gamma^{*}\left(1 / z^{*}\right)=i v\left(\frac{1}{z} \hat{f}_{-1}\left(z^{2}, T\right)-z \hat{f}_{0}\left(z^{2}, T\right)\right)$. 
Thus (3.41) yields, to $O(\varepsilon)$,

$q_{n}(t)=\frac{1}{2 \pi i} \int_{|\zeta|=1} \zeta^{2 n-1} \mathrm{e}^{-i \omega\left(\zeta^{2}\right) t} \hat{q}\left(\zeta^{2}, 0\right) \mathrm{d} \zeta+\frac{1}{2 \pi} \int_{L_{1}+L_{4}} \zeta^{2 n} \mathrm{e}^{-i \omega\left(\zeta^{2}\right) t}\left(\frac{1}{\zeta} \hat{f}_{-1}\left(\zeta^{2}, T\right)-\zeta \hat{f}_{0}\left(\zeta^{2}, T\right)\right) \mathrm{d} \zeta$,

where the integrals are taken in Cauchy's principal value sense. Now note that the contour $L_{1} \cup L_{4}$ can be deformed to $\partial D_{+ \text {in }}$ by Cauchy's theorem. Performing the change of variable $\zeta^{2}=z$, we then obtain that the linear limit of (3.41) coincides with the solution of the IBVP for the DLS on the natural numbers, namely (2.24).

Continuum limit. Reinstating the lattice spacing $h$, it is easy to show that the Lax pair for the NLS is the continuum limit of that for the AL system as $h \rightarrow 0[4,6]$. The continuum limit is formally obtained by writing the solution of the discrete case as $Q_{n}(t)=h q(n h, t)$ and $P_{n}(t)=h p(n h, t)$. Then for $z=\mathrm{e}^{i k h}$, the Lax pair (3.2) becomes

$$
\begin{aligned}
& \frac{\mu_{n+1}-\mu_{n}}{h}-i k\left[\sigma_{3}, \mu_{n}\right]=\mathrm{Q}_{n}(t) \mu_{n}+O\left(h^{2}\right), \\
& \dot{\mu}_{n}+i \omega(k)\left[\sigma_{3}, \mu_{n}\right]=\mathrm{H}_{n}(t, k) \mu_{n}+O(h),
\end{aligned}
$$

where now $\omega(k)=(1-\cos 2 k h) / h^{2}$, with $\mu_{n}=\mu(n h, t, k), q_{n}=q(n h, t)$ and $p_{n}=p(n h, t)$ for brevity, and where

$$
\mathrm{H}_{n}(t, k)=i\left(\begin{array}{cc}
-q_{n} p_{n-1} & \left(q_{n}-q_{n-1}\right) / h+i k\left(q_{n}+q_{n-1}\right) \\
-\left(p_{n}-p_{n-1}\right) / h+i k\left(p_{n}+p_{n-1}\right) & q_{n-1} p_{n}
\end{array}\right) .
$$

Correspondingly, the Jost solutions are obtained from (3.25), for example,

$\mu_{n}^{(1)}(k, t)=\mathrm{I}+h \sum_{m=0}^{n-1} \mathrm{e}^{i k h(n-m) \hat{\sigma}_{3}}\left(\mathrm{Q}_{m}(t) \mu_{m}^{(1)}(k, t)\right)+\int_{0}^{t} \mathrm{e}^{i\left[n k h-\omega(k)\left(t-t^{\prime}\right)\right] \hat{\sigma}_{3}}\left(\mathrm{H}\left(0, k, t^{\prime}\right) \mu_{0}^{(1)}\left(k, t^{\prime}\right)\right) \mathrm{d} t^{\prime}$.

As $h \rightarrow 0$ with $x=n h$ fixed, we have $\omega(k) \rightarrow 2 k^{2}$, together with $\mathrm{H}_{n}(t, k) \rightarrow \mathrm{H}(x, t, k)$ and $\mu_{n}^{(j)}(k, t) \rightarrow \mu^{(j)}(x, t, k), j=1,2,3$, where $\mu^{(j)}(x, t, k)$ are the Jost solutions for the IBVP of the NLS, namely (5.38). Note also that $C_{n} \rightarrow 1$ as $h \rightarrow 0$. Hence, in the continuum limit, the solution of the IBVP for the IDNLS becomes exactly that of the IBVP for NLS.

The result can also be verified directly via the continuum limit of the solution (3.41). Explicitly, since $C_{0}=1+O\left(h^{2}\right)$, as $h \rightarrow 0$ we have

$$
\begin{aligned}
Q_{n}(t) & =-\frac{1}{2 \pi i} \int_{|\zeta|=1} \zeta^{2 n} \mathrm{e}^{-2 i \omega(\zeta) t} \gamma(\zeta)\left(\mathrm{M}_{n+1}^{+}(\zeta, t)\right)_{11} \mathrm{~d} \zeta \\
& +\frac{v}{2 \pi i} \int_{L_{1}+L_{4}} \zeta^{2 n} \mathrm{e}^{-2 i \omega(\zeta) t} \Gamma^{*}\left(1 / \zeta^{*}\right)\left(\mathrm{M}_{n+1}^{+}(\zeta, t)\right)_{11} \mathrm{~d} \zeta+\frac{v}{2 \pi i} \int_{L_{2}}|\gamma(\zeta)|^{2}\left(\mathrm{M}_{n+1}^{+}(\zeta, t)\right)_{12} \frac{\mathrm{d} \zeta}{\zeta^{2}}+O\left(h^{2}\right) .
\end{aligned}
$$

The oriented contour $L_{1} \cup L_{4}$ can be deformed onto $|\zeta|=1$ since the corresponding integrand is analytic in $D_{- \text {in }}$. In terms of $q(n h, t)=Q_{n}(t) / h$, and performing the substitution $\zeta=\mathrm{e}^{i k h}$, we then have

$$
\begin{aligned}
& q(n h, t)=-\frac{h}{\pi} \int_{-\pi / h}^{\pi / h} \mathrm{e}^{2 i(n k h-\omega(k) t)} \gamma(k)\left(\mathrm{M}_{n+1}^{+}(k, t)\right)_{11} \mathrm{e}^{i k h} \mathrm{~d} k \\
&+\frac{h}{\pi} \int_{-\pi / h}^{\pi / h} v \mathrm{e}^{2 i(n k h-\omega(k) t)} \Gamma^{*}\left(k^{*}\right)\left(\mathrm{M}_{n+1}^{+}(k, t)\right)_{11} \mathrm{e}^{i k h} \mathrm{~d} k-\frac{h}{\pi} \int_{\pi / 2 h}^{\pi / h} v|\gamma(k)|^{2}\left(\mathrm{M}_{n+1}^{+}(k, t)\right)_{12} \mathrm{e}^{-i k h} \mathrm{~d} k \\
&-\frac{h}{\pi} \int_{0}^{\pi / 2 h} v|\gamma(-k)|^{2}\left(\mathrm{M}_{n+1}^{+}(-k, t)\right)_{12} \mathrm{e}^{i k h} \mathrm{~d} k .
\end{aligned}
$$


Now note that, since $\mathrm{e}^{-4 i k^{2} t} \Gamma^{*}\left(k^{*}\right)\left(\mathrm{M}_{n+1}^{+}(x, k, t)\right)_{11}$ is analytic and bounded for (Re $k \in$ $[-\pi / h, 0]) \wedge(\operatorname{Im} k>0)$ [which becomes $\mathbb{C}_{\text {II }}$ in the limit $h \rightarrow 0$ ], the portion of the corresponding integral on the negative real axis can be deformed onto the positive imaginary axis. Then, taking the continuum limit of all the integrals in (3.47) we obtain that $q(n h, t)$ coincides with the solution of the IBVP for the NLS, namely (5.45), in the limit $h \rightarrow 0$.

Remarks. A few comments are now in order:

- Equation (3.41) provides the Ehrenpreis [18, 37, 32] representation for the solution of the IBVP for the IDNLS, in analogy with Ref. [27] in the continuum limit.

- One can now use (3.41) as a starting point to formally prove that the function $q(x, t)$ given by the reconstruction formula satisfies (1.2) as well as the initial-boundary conditions, using the dressing method, as in Ref. [29] in the continuum limit.

- In the continuum problem, the location of the jumps is the union of the jumps for the scattering problem in the linear case and those of its adjoint. In the discrete problem, however, this is not the case. Indeed, the extra jump along the imaginary axis arises as a consequence of the rescaling $z \rightarrow z^{2}$ when going from the linear to the nonlinear case.

- The scattering matrix $\mathrm{S}(z, T)$ involves $T$ explicitly. In Appendix E, however, we show that the solution of the IBVP for the AL system on the naturals does not depend on future values of the boundary datum.

- With the due modifications, the method presented here can also be used to solve the IBVP for all members of the Ablowitz-Ladik hierarchy. Moreover, the method can be generalized to any integrable differential-difference evolution equation.

\section{Elimination of the unknown boundary datum, linearizable BCs and soliton solutions}

\subsection{Elimination of the unknown boundary datum}

The scattering matrix $\mathrm{S}(z, T)$ depends on the both the known and the unknown boundary datum. In the linear problem, it was possible to overcome this difficulty by making use of the fact that the transformation $z \rightarrow 1 / z$ leaves the transforms of the boundary data unchanged. In the nonlinear problem, however, the matrix $\mathrm{S}(z, T)$ is not invariant under this transformation, because it is defined in terms of the eigenfunction $\mu_{n}^{(1)}(z, t)$, which is not invariant under $z \rightarrow 1 / z$. As in the continuum case [28], the determination of the unknown boundary datum in terms of the known initial-boundary conditions is in general a nontrivial issue.

For linearizable BCs it is possible to express the RHP only in terms of the initial data, as we show in section 4.2. This is not possible for generic BCs, however. In this case one must solve a coupled system of nonlinear ordinary differential equations (ODEs) to obtain simultaneously the unknown boundary datum $q_{-1}(t)$ as well as scattering coefficients $A(z, T)$ and $B(z, T)$, as we show next.

The boundary data enters the RHP only via the ratio $R(z, T)=B(z, T) / A(z, T)$ appearing in $\Gamma(z)$. Recalling (3.1) and (3.28), we have $\mathrm{S}(z, t)=\tilde{\Phi}^{-1}(z, t) \mathrm{e}^{-i \omega(z) t \sigma_{3}}$, where the matrix

$$
\tilde{\Phi}(z, t)=\Phi_{0}^{(1)}(z, t)=\left(\begin{array}{cc}
e^{-i \omega(z) t} A^{*}\left(1 / z^{*}\right) & -v e^{-i \omega(z) t} B^{*}\left(1 / z^{*}\right) \\
-e^{i \omega(z) t} B(z) & e^{i \omega(z) t} A(z)
\end{array}\right),
$$

satisfies the $t$-part of the Lax pair (A.5) for $n=0$, namely:

$$
\dot{\tilde{\Phi}}=\left(-i \omega(z) \sigma_{3}+\mathrm{H}_{0}(z, t)\right) \tilde{\Phi},
$$

together with the initial condition $\tilde{\Phi}(z, 0)=\mathrm{I}$. 
The term $\mathrm{H}_{0}(z, t)$ in (A.6b) contains $q_{-1}(t)$, of course. Note however that using (3.33) with $n=0$, we can express $q_{-1}(t)$ in terms of $\mu_{0}^{(1)}(z, t)$ :

$$
q_{-1}(t)=\lim _{z \rightarrow 0}\left(\mu_{0}^{(1)}(z, t)\right)_{12} / z \quad z \in D_{- \text {in }} .
$$

The simultaneous solution of (4.1) and (4.3) provides the unknown boundary datum as well as the auxiliary spectral functions $A(z, t)$ and $B(z, t)$, allowing one to completely define the RHP and therefore we also obtain the solution of the inverse problem. Note that this procedure is significantly simpler than that requried to obtain the generalized Dirichlet-to-Neumann map in the continuum case [28].

\subsection{Linearizable boundary conditions}

Like in the continuum case, there is a class of BCs, called linearizable, for which it is possible to obtain the unknown boundary datum via only algebraic manipulations of the global relation.

Recall that $A(z, t)$ and $B(z, t)$ are given in terms of $\Phi(z, t)=\mu_{0}^{(1)} \mathrm{e}^{-i \omega(z) t \sigma_{3}}$ by (4.1) which solves the ODE (4.2) together with the initial condition $\tilde{\Phi}(z, 0)=\mathrm{I}$. Since $\omega(1 / z)=\omega(z)$, the matrix $\tilde{\Phi}(1 / z, t)$ satisfies equations identical to $(4.2)$ except that $\mathrm{H}_{0}(z, t)$ is replaced by $\mathrm{H}_{0}(1 / z, t)$. If there exists a time-independent matrix $\mathrm{N}(z)$ such that

$$
\mathrm{N}(z)\left(-i \omega(z) \sigma_{3}+\mathrm{H}_{0}(z, t)\right)=\left(-i \omega(z) \sigma_{3}+\mathrm{H}_{0}(1 / z, t)\right) \mathrm{N}(z),
$$

it is then easy to show that

$$
\tilde{\Phi}(1 / z, t)=\mathrm{N}(z) \tilde{\Phi}(z, t) \mathrm{N}(z)^{-1} .
$$

A necessary condition for (4.4) to be satisfied is obviously that $\operatorname{det}\left[-i \omega(z) \sigma_{3}+\mathrm{H}_{0}(z, t)\right]=$ $\left[\left(z^{2}-1 / z^{2}\right)\left(q_{0} p_{-1}-q_{-1} p_{0}\right)\right]^{2}$ be invariant under the transformation $z \rightarrow 1 / z$. In turn, for this condition to be satisfied one needs

$$
q_{0} p_{-1}-q_{-1} p_{0}=0 \text {. }
$$

In the reduction $p_{n}(t)=v q_{n}^{*}(t)$ to IDNLS, (4.6) is satisfied by the discrete analogue of homogeneous Robin BCs:

$$
q_{-1}-\chi q_{0}=0, \quad \chi \in \mathbb{R} .
$$

These BCs had been previously identified via algebraic methods [31]. For the BCs (4.7), we can solve the system (4.4) for $\mathrm{N}(z)$, obtaining $N_{12}=N_{21}=0$ and $N_{11}=f(z) N_{22}$, where

$$
f(z)=\frac{1-\chi z^{2}}{z^{2}-\chi} \text {. }
$$

Recalling (4.5), we then find the following symmetries for the scattering data:

$$
A^{*}\left(z^{*}, T\right)=A^{*}\left(1 / z^{*}, T\right), \quad B^{*}\left(z^{*}, T\right)=f(z) B^{*}\left(1 / z^{*}, T\right) .
$$

Note that $\mathrm{N}(z)$ is not invertible for $z= \pm \chi^{1 / 2}, \pm \chi^{-1 / 2}$. However, (4.8) is still valid at such values of $z$. Indeed, since $\tilde{\Phi}(z, t)$ solves $(4.2)$, writing a Neumann series for $\tilde{\Phi}(z, t)$ one finds $\tilde{\Phi}\left( \pm \chi^{1 / 2}, t\right)_{12}=0$, which implies that $B^{*}\left( \pm \chi^{-1 / 2}, t\right)=0$. As a consequence, since $A^{*}\left(1 / z^{*}, T\right)$ and $B^{*}\left(1 / z^{*}, T\right)$ are analytic for $z \in \mathbb{C}^{[\emptyset]}$, we can conclude that the limit as $z \rightarrow \pm \chi^{1 / 2}$ of the product $f(z) B^{*}\left(1 / z^{*}, T\right)$ exists and is finite.

The above properties now allow $\Gamma^{*}\left(1 / z^{*}\right)$ to be expressed in terms of the known functions, $a(z)$ and $b(z)$. For simplicity, we consider the case in which no discrete spectrum is present. Consider first the case $T=\infty$. The global relation in this case is simply given by (3.45). Replacing $1 / z$ by $z$ and using (4.8), we obtain

$$
A^{*}\left(1 / z^{*}\right)=\frac{a^{*}\left(z^{*}\right) d(z)}{\Delta(1 / z)}, \quad B^{*}\left(1 / z^{*}\right)=\frac{f(1 / z) b^{*}\left(z^{*}\right) d(z)}{\Delta(1 / z)} \quad z \in D_{+ \text {in }},
$$


where

$$
\Delta(z)=a(1 / z) a^{*}\left(1 / z^{*}\right)-v f(z) b(1 / z) b^{*}\left(1 / z^{*}\right) .
$$

As a result, we can express the ratio $R(z, T)=B^{*}\left(1 / z^{*}, T\right) / A^{*}\left(1 / z^{*}, T\right)$ as

$$
R(z, T)=f(1 / z) \frac{b^{*}\left(z^{*}\right)}{a^{*}\left(z^{*}\right)} \quad z \in D_{+ \text {in }},
$$

and we therefore obtain $\Gamma^{*}\left(1 / z^{*}\right)$ only in terms of known spectral functions. Now consider the case $T<\infty$. The global relation in this case is (3.44). Replacing $1 / z$ by $z$ in (3.44) and using the symmetry (4.8) as before, we obtain

$$
R(z, T)=f(1 / z) \frac{b^{*}\left(z^{*}\right)}{a^{*}\left(z^{*}\right)}+v \mathrm{e}^{2 i \omega(z) T} \frac{f(1 / z) G(1 / z, T)}{a^{*}\left(z^{*}\right) A^{*}\left(z^{*}, T\right)}, \quad z \in D_{\text {+in }} .
$$

We therefore see that the difference from the case $T=\infty$ is simply the appearance of an additional term in the RHS of (4.10). In Appendix E, however, we show that the second term in the RHS of (4.11) does not affect the solution of the IBVP for the IDNLS. Hence, even in the case $T<\infty$, we can use (4.10) in the RHP (3.36).

\subsection{Discrete spectrum and soliton solutions}

Equations (3.35) imply that when the functions $a(z)$ and $d(z)$ possess zeros the matrices $\mathrm{M}_{n}^{ \pm}(z, t)$ are only meromorphic functions in $D_{+}$and $D_{-}$, respectively. As a consequence, the RHP (3.36) formulated becomes singular. As in the IVP, however, it can be converted to a regular RHP by taking into account the appropriate residue relations. We assume that these discrete eigenvalues are all simple. More precisely, we assume that:

- $a(z)$ has simples zeros in $D_{\text {-in }}$. We label such zeros $\pm z_{j}$ for $j=1, \ldots, J$;

- $d(z)$ has simple zeros in $D_{+ \text {in }}$. We label such zeros $\pm \lambda_{j}$ for $j=1, \ldots, J^{\prime}$.

We also assume that there are no zeros on the boundaries of these domains and that there are no common zeros of $a(z)$ and $d(z)$ in $D_{+ \text {in }}$.

The fact that the zeros of $a(z)$ and $d(z)$ always appear in opposite pairs is a trivial consequence of $a(z)$ and $d(z)$ both being even functions of $z$ [cf. Appendix D]. Also, the symmetry $p_{n}(t)=v q_{n}(t)$ of the potentials implies that, corresponding to these zeros, there is an equal number of zeros of $a^{*}\left(1 / z^{*}\right)$ and $d^{*}\left(1 / z^{*}\right)$ in $D_{+ \text {out }}$ and $D_{- \text {out }}$, respectively, which we denote respectively by $\bar{z}_{j}=1 / z_{j}^{*}$ and $\bar{\lambda}_{j}=1 / \lambda_{j}^{*}$. Thus, discrete eigenvalues in the IBVP can appear in two different kinds of quartets, namely,

$$
\left\{ \pm z_{j}, \pm \bar{z}_{j}\right\}_{j=1}^{J}, \quad\left\{ \pm \lambda_{j}, \pm \bar{\lambda}_{j}\right\}_{j=1}^{J^{\prime}} .
$$

Similarly to the IVP, from (3.27) and (3.36) we find the following residue relations;

$$
\begin{array}{ll}
\operatorname{Res}_{z=z_{j}}\left[\mathrm{M}_{n}^{(-, R)}\right]=a_{j} \mathrm{M}_{n}^{(-, L)}\left(z_{j}\right), & \operatorname{Res}_{z=\bar{z}_{j}}\left[\mathrm{M}_{n}^{(+, L)}\right]=\bar{a}_{j} \mathrm{M}_{n}^{(+, R)}\left(\bar{z}_{j}\right), \\
\operatorname{Res}_{z=\lambda_{j}}\left[\mathrm{M}_{n}^{(+, R)}\right]=d_{j} \mathrm{M}_{n}^{(+, L)}\left(\lambda_{j}\right), & \operatorname{Res}_{z=\bar{\lambda}_{j}}\left[\mathrm{M}_{n}^{(-, L)}\right]=\bar{d}_{j} \mathrm{M}_{n}^{(-, R)}\left(\bar{\lambda}_{j}\right),
\end{array}
$$

where

$$
\begin{array}{ll}
a_{j}=K_{j} z_{j}^{2 n} \mathrm{e}^{-2 i \omega\left(z_{j}\right) t}, \quad \bar{a}_{j}=\bar{K}_{j} \bar{z}_{j}^{-2 n} \mathrm{e}^{2 i \omega\left(\bar{z}_{j}\right) t}, \quad d_{j}=\Lambda_{j} \lambda_{j}^{2 n} \mathrm{e}^{-2 i \omega\left(\lambda_{j}\right) t}, \quad \bar{d}_{j}=\bar{\Lambda}_{j} \bar{\lambda}_{j}^{-2 n} \mathrm{e}^{2 i \omega\left(\bar{\lambda}_{j}\right) t}, \\
K_{j}=1 /\left(\dot{a}\left(z_{j}\right) b\left(z_{j}\right)\right), \quad \Lambda_{j}=v B^{*}\left(\bar{\lambda}_{j}\right) /\left(a\left(\lambda_{j}\right) \dot{d}\left(\lambda_{j}\right)\right), \quad \bar{K}_{j}=\left(-z_{j}^{*}\right)^{-2} v K_{j}, \quad \bar{\Lambda}_{j}=\left(-\lambda_{j}^{*}\right)^{-2} v \Lambda_{j} .
\end{array}
$$

and as customary $K_{j}, \Lambda_{j}, \bar{K}_{j}$ and $\bar{\Lambda}_{j}$ are referred to as norming constants. Note that since $b(z)$ and $B^{*}\left(1 / z^{*}\right)$ are odd functions of $z$ [cf. Appendix D], the norming constants $K_{j}$ at $z= \pm z_{j}$ are identical, and the same follows for $\Lambda_{j}$ at $z= \pm \lambda_{j}$. 
The RHP is now solved by removing the singularities, which is done by subtracting the residue contributions at the poles. As usual, the solution of the RHP then has additional terms compared to the case of no poles (3.37), and is given by

$$
\begin{aligned}
\mathrm{M}_{n}(z, t)=\mathrm{I}+\frac{1}{2 \pi i} \int_{L} \mathrm{M}_{n}^{+}(\zeta, t) \frac{\mathrm{J}_{n}(\zeta, t)}{\zeta-z} \mathrm{~d} \zeta,+\sum_{j=1}^{2 J}\left(\frac{1}{z-z_{j}} \operatorname{Res}_{z=z_{j}}\left[\mathrm{M}_{n}^{-}(z)\right]+\frac{1}{z-\bar{z}_{j}} \operatorname{Res}_{z=\bar{z}_{j}}\left[\mathrm{M}_{n}^{+}(z)\right]\right) \\
+\sum_{j=1}^{2 J^{\prime}}\left(\frac{1}{z-\lambda_{j}} \operatorname{Res}_{z=\lambda_{j}}\left[\mathrm{M}_{n}^{+}(z)\right]+\frac{1}{z-\bar{\lambda}_{j}} \operatorname{Res}_{z=\bar{\lambda}_{j}}\left[\mathrm{M}_{n}^{-}(z)\right]\right),
\end{aligned}
$$

where we defined $z_{j+J}=-z_{j}$ for $j=1, \ldots, J$ and $\lambda_{j+J^{\prime}}=-\lambda_{j}$ for $j=1, \ldots, J^{\prime}$. From the asymptotic expansion of (4.13) and the symmetries (D.8), we then obtain the reconstruction formula:

$q_{n}(t)=-2 \sum_{j=1}^{J} z_{j}^{2 n} \mathrm{e}^{-2 i \omega\left(z_{j}\right) t} K_{j} \mathrm{M}_{n+1,11}^{-}\left(z_{j}\right)-2 \sum_{j=1}^{J^{\prime}} \lambda_{j}^{2 n} \mathrm{e}^{-2 i \omega\left(\lambda_{j}\right) t} \Lambda_{j} \mathrm{M}_{n+1,11}^{+}\left(\lambda_{j}\right)+\tilde{q}_{n}(t)$,

where $\tilde{q}_{n}(t)$ is given by (3.41).

In the reflectionless case with $v=-1$, we obtain the soliton solution solving the following algebraic system of equations for $\mathrm{M}_{n+1,11}^{-}\left(z_{j}\right)$ and $\mathrm{M}_{n+1,11}^{+}\left(\lambda_{j}\right)$ :

$$
\begin{aligned}
& \mathrm{M}_{n, 11}^{-}\left(z_{l}\right)=1+\sum_{j=1}^{J} \bar{a}_{j}\left(\frac{1}{z_{l}-\bar{z}_{l}}-\frac{1}{z_{l}+\bar{z}_{j}}\right) \mathrm{M}_{n, 12}^{+}\left(\bar{z}_{j}\right)+\sum_{j=1}^{J^{\prime}} \bar{d}_{j}\left(\frac{1}{z_{l}-\bar{\lambda}_{j}}-\frac{1}{z_{l}+\bar{\lambda}_{j}}\right) \mathrm{M}_{n, 12}^{-}\left(\bar{\lambda}_{j}\right) \\
& \mathrm{M}_{n, 12}^{+}\left(\bar{z}_{l}\right)=\sum_{j=1}^{J} a_{j}\left(\frac{1}{\bar{z}_{l}-z_{j}}+\frac{1}{\bar{z}_{l}+z_{j}}\right) \mathrm{M}_{n, 11}^{-}\left(z_{j}\right)+\sum_{j=1}^{J^{\prime}} d_{j}\left(\frac{1}{\bar{z}_{l}-\lambda_{j}}+\frac{1}{\bar{z}_{l}+\lambda_{j}}\right) \mathrm{M}_{n, 11}^{+}\left(\lambda_{j}\right), \\
& \mathrm{M}_{n, 11}^{+}\left(\lambda_{l}\right)=1+\sum_{j=1}^{J} \bar{a}_{j}\left(\frac{1}{\lambda_{l}-\bar{z}_{l}}-\frac{1}{\lambda_{l}+\bar{z}_{j}}\right) \mathrm{M}_{n, 12}^{+}\left(\bar{z}_{j}\right)+\sum_{j=1}^{J^{\prime}} \bar{d}_{j}\left(\frac{1}{\lambda_{l}-\bar{\lambda}_{j}}-\frac{1}{\lambda_{l}+\bar{\lambda}_{j}}\right) \mathrm{M}_{n, 12}^{-}\left(\bar{\lambda}_{j}\right), \\
& \mathrm{M}_{n, 12}^{-}\left(\bar{\lambda}_{l}\right)=\sum_{j=1}^{J} a_{j}\left(\frac{1}{\bar{\lambda}_{l}-z_{j}}+\frac{1}{\bar{\lambda}_{l}+z_{j}}\right) \mathrm{M}_{n, 11}^{-}\left(z_{j}\right)+\sum_{j=1}^{J^{\prime}} d_{j}\left(\frac{1}{\bar{\lambda}_{l}-\lambda_{j}}+\frac{1}{\bar{\lambda}_{l}+\lambda_{j}}\right) \mathrm{M}_{n, 11}^{+}\left(\lambda_{j}\right) .
\end{aligned}
$$

For a single quartet $\left\{ \pm z_{1}, \pm \bar{z}_{1}\right\}$, the solution of the above system with $J=1$ and $J^{\prime}=0$ yields the one-soliton solution of the IDNLS as

$$
q_{n}(t)=\mathrm{e}^{2 i[(n+1) \beta+2 w t+\phi]} \sinh (2 \alpha) \operatorname{sech}[2((n+1) \alpha-v t-\delta)],
$$

where $z_{1}=\mathrm{e}^{\alpha+i \beta}$ and

$$
\begin{aligned}
& w=\cosh (2 \alpha) \cos (2 \beta)-1, \quad v=\sinh (2 \alpha) \sin (2 \beta), \\
& \delta=\frac{1}{2} \log (\sinh (2 \alpha))-\frac{1}{2} \log \left|K_{1}\right|+\log \left|z_{1}\right|, \quad \phi=\frac{\pi}{2}-\arg z_{1}+\frac{1}{2} \arg K_{1} .
\end{aligned}
$$

The soliton solution corresponding to a single quartet $\left\{ \pm \lambda_{1}, \pm \bar{\lambda}_{1}\right\}$ has an identical functional representation, which also coincides with the well-known one-soliton solution in the IVP.

Note that the norming constants $\Lambda_{j}$ contain the unknown scattering datum $q_{-1}(t)$ through the spectral functions $A(z, t)$ and $B(z, t)$. In general, this datum must be obtained by solving a nonlinear system of ODEs, as explained previously. In the case of linearizable BCs, however, $\Lambda_{j}$ can be expressed only in terms of known scattering data. In particular, with $T=\infty$, the global relation implies

$$
\Lambda_{j}=f\left(1 / \lambda_{j}\right) b^{*}\left(\lambda_{j}^{*}\right) /\left[a\left(\lambda_{j}\right) \dot{\Delta}\left(1 / \lambda_{j}\right)\right],
$$

where $\Delta(z)$ was defined in (4.9). This result can then be used in the residue relations (4.12b). Equation (4.16) is a consequence of the fact that $d(z)$ and $\Delta(1 / z)$ have the same set of zeros in $D_{+ \text {in }}$, which in turn can be easily proved considering the analyticity of $A^{*}\left(1 / z^{*}\right)$ and $B^{*}\left(1 / z^{*}\right)$ with (4.9). 


\section{Continuum: linear and nonlinear Schrödinger equations}

In order to compare the solution of the IBVP in the discrete case to its continuum limit, and to appreciate the differences between the method for discrete problems and its continuum counterpart, here we briefly review the solution of IBVPs for the linear Schrödinger (LS) equation and the nonlinear Schrödinger (NLS) equation:

$$
i \frac{\partial q}{\partial t}+\frac{\partial^{2} q}{\partial x^{2}}-2 v|q|^{2} q=0
$$

(with $v=0, \pm 1$ denoting respectively the linear, defocusing and focusing cases), to which (1.1) and (1.2) reduce to in the limit $h \rightarrow 0$. Note that, even though the IVP for (5.1) was solved in the early days of integrable systems for both vanishing [44] and nonzero [45] BCs, the IBVP on the half line was solved only recently [29]. Also, even though the IVP for the vector generalization of (5.1) was also solved early on in the case of vanishing BCs [34], the analogue problem with nonzero BCs was also only recently solved [38].

Linear Schrödinger equation: IVP and IBVP via Fourier methods. Consider first the initial value problem for the LS equation with $x \in \mathbb{R}, t>0$ and $q(x, 0)$ given. For simplicity we assume that $q(x, 0)$ belongs to the Schwartz class, which we denote by $\mathcal{S}(\mathbb{R})$. The IVP is trivially solved using the Fourier transform pair, defined as

$$
\hat{q}(k, t)=\int_{-\infty}^{\infty} \mathrm{e}^{-i k x} q(x, t) \mathrm{d} x, \quad q(x, t)=\frac{1}{2 \pi} \int_{-\infty}^{\infty} \mathrm{e}^{i k x} \hat{q}(k, t) \mathrm{d} k .
$$

Use of (5.2) yields the solution of the IVP as

$$
q(x, t)=\frac{1}{2 \pi} \int_{-\infty}^{\infty} \mathrm{e}^{i\left(k x-k^{2} t\right)} \hat{q}(k, 0) \mathrm{d} k .
$$

Now consider the IBVP for the LS equation on the half line with Dirichlet BCs; i.e., $x>0$, $t>0$ and with $q(x, 0) \in \mathcal{S}\left(\mathbb{R}^{+}\right)$and $q(0, t) \in C\left(\mathbb{R}^{+}\right)$given. Employing the sine transform pair

$$
\hat{q}^{(s)}(k, t)=\int_{0}^{\infty} \sin (k x) q(x, t) \mathrm{d} x, \quad q(x, t)=\frac{2}{\pi} \int_{0}^{\infty} \sin (k x) \hat{q}^{(s)}(k, t) \mathrm{d} k,
$$

yields the solution of the IBVP as

$$
q(x, t)=\frac{2}{\pi} \int_{0}^{\infty} \mathrm{e}^{-i k^{2} t} \sin (k x) \hat{q}^{(s)}(k, 0) \mathrm{d} k+\frac{1}{\pi} \int_{0}^{\infty} \mathrm{e}^{-i k^{2} t} \sin (k x) \hat{g}(k, t) \mathrm{d} k,
$$

where

$$
\hat{g}(k, t)=2 i k \int_{0}^{t} \mathrm{e}^{i k^{2} t^{\prime}} q\left(0, t^{\prime}\right) \mathrm{d} t^{\prime}
$$

\subsection{Linear Schrödinger equation: IVP and IBVPs via spectral methods}

An algorithmic method to obtain the Lax pair of linear PDEs was given in Ref. [25]. However, one can also obtain the Lax pair for the LS equation via the linear limit of the Lax pair of the NLS equation, namely (A.2). Let $\mathrm{Q}=O(\varepsilon)$ and take $\Phi(x, t, k)=\mathbf{v}(x, t, k)$ to be a twocomponent vector. To leading order it is $\mathbf{v}(x, t, k)=\mathrm{e}^{i\left(k x-2 k^{2} t\right) \hat{\sigma}_{3}} \mathbf{v}_{o}$, where $\mathbf{v}_{o}=\left(v_{1, o}, v_{2, o}\right)$ is an arbitrary constant vector. Choosing $v_{2, o}=1$ and substituting into the RHS of (A.2a) then yields the following equations for $\mu(x, t, k)=\mathrm{e}^{i\left(k x-2 k^{2} t\right)} v_{1}(x, t, k)$ up to $O\left(\varepsilon^{2}\right)$ terms:

$$
\mu_{x}-i k^{\prime} \mu=q, \quad \mu_{t}+i k^{2} \mu=i q_{x}-k^{\prime} q,
$$

where $k^{\prime}=2 k$. One can now verify that enforcing the compatibility of (5.7) yields the LS equation. Hereafter, for convenience, we will omit the primes. 
Initial value problem. Introduce a modified eigenfunction $\psi(x, t, k)=\mathrm{e}^{-i\left(k x-k^{2} t\right)} \mu(x, t, k)$, which satisfies the simplified Lax pair

$$
\psi_{x}=\mathrm{e}^{-i\left(k x-k^{2} t\right)} q, \quad \psi_{t}=\mathrm{e}^{-i\left(k x-k^{2} t\right)}\left(i q_{x}-k q\right) .
$$

It is then easy to obtain the solutions of (5.7) which decay as $x \rightarrow \pm \infty$ respectively as:

$$
\mu^{(1)}(x, t, k)=\int_{-\infty}^{x} \mathrm{e}^{i k\left(x-x^{\prime}\right)} q\left(x^{\prime}, t\right) \mathrm{d} x^{\prime}, \quad \mu^{(2)}(x, t, k)=-\int_{x}^{\infty} \mathrm{e}^{i k\left(x-x^{\prime}\right)} q\left(x^{\prime}, t\right) \mathrm{d} x^{\prime} .
$$

Note that $\mu^{(1,2)}(x, t, k)$ are analytic for $\operatorname{Im} k \gtrless 0$, respectively Also, on $\operatorname{Im} k=0$ it is

$$
\mu^{(1)}(x, t, k)-\mu^{(2)}(x, t, k)=\mathrm{e}^{i k x} \hat{q}(k, t)=\mathrm{e}^{i\left(k x-k^{2} t\right)} \hat{q}(k, 0),
$$

where $\hat{q}(k, t)$ is the Fourier transform of $q(x, t)$ :

$$
\hat{q}(k, t)=\int_{-\infty}^{\infty} \mathrm{e}^{-i k x^{\prime}} q\left(x^{\prime}, t\right) \mathrm{d} x^{\prime} .
$$

Also, $\mu^{(1,2)}(x, t, k)=O(1 / k)$ as $k \rightarrow \infty$ in their respective half planes. Thus (5.9) defines a scalar RHP which is trivially solved via the standard Cauchy projectors $P^{ \pm}$over the real line:

$$
\mu(x, t, k)=\frac{1}{2 \pi i} \int_{-\infty}^{\infty} \mathrm{e}^{i\left(k^{\prime} x-k^{\prime 2} t\right)} \frac{\hat{q}\left(k^{\prime}, 0\right)}{k^{\prime}-k} \mathrm{~d} k^{\prime} .
$$

Inserting (5.11) into (5.7) then yields (5.3) as the solution of the IVP.

Initial-boundary value problems. We now consider the IBVP for the LS equation on the half line. Define simultaneous solutions of both the $x$-part and the $t$-part of the Lax pair:

$$
\mu^{(j)}(x, t, k)=\int_{\left(x_{j}, t_{j}\right)}^{(x, t)} \mathrm{e}^{i k\left(x-x^{\prime}\right)-i k^{2}\left(t-t^{\prime}\right)}\left[q\left(x^{\prime}, t^{\prime}\right) \mathrm{d} x^{\prime}+\left(i q_{x^{\prime}}\left(x^{\prime}, t^{\prime}\right)-k q\left(x^{\prime}, t^{\prime}\right)\right) \mathrm{d} t^{\prime}\right] .
$$

In particular, consider the three eigenfunctions $\mu^{(j)}(x, t, k), j=1,2,3$, defined by the choices $\left(x_{1}, t_{1}\right)=(0,0),\left(x_{2}, t_{2}\right)=(\infty, t)$ and $\left(x_{3}, t_{3}\right)=(0, T)$ :

$$
\begin{aligned}
& \mu^{(1)}(x, t, k)=\int_{0}^{x} \mathrm{e}^{i k\left(x-x^{\prime}\right)} q\left(x^{\prime}, t\right) \mathrm{d} x^{\prime}+\int_{0}^{t} \mathrm{e}^{i k x-i k^{2}\left(t-t^{\prime}\right)}\left(i q_{x}\left(0, t^{\prime}\right)-k q\left(0, t^{\prime}\right)\right) \mathrm{d} t^{\prime}, \\
& \mu^{(2)}(x, t, k)=-\int_{x}^{\infty} \mathrm{e}^{i k\left(x-x^{\prime}\right)} q\left(x^{\prime}, t\right) \mathrm{d} x^{\prime}, \\
& \mu^{(3)}(x, t, k)=\int_{0}^{x} \mathrm{e}^{i k\left(x-x^{\prime}\right)} q\left(x^{\prime}, t\right) \mathrm{d} x^{\prime}-\int_{t}^{T} \mathrm{e}^{i k x-i k^{2}\left(t-t^{\prime}\right)}\left(i q_{x}\left(0, t^{\prime}\right)-k q\left(0, t^{\prime}\right)\right) \mathrm{d} t^{\prime} .
\end{aligned}
$$

Note that $\mu^{(2)}$ coincides with the eigenfunction in the IVP. As for $\mu^{(1)}$ and $\mu^{(3)}$, they are entire functions of $k$. These eigenfunctions have the following domains of analyticity and boundedness:

$$
\mu^{(1)}: k \in \mathbb{C}_{\mathrm{II}}, \quad \mu^{(2)}: k \in \mathbb{C}_{\mathrm{III}+\mathrm{IV}}, \quad \mu^{(3)}: k \in \mathbb{C}_{\mathrm{I}},
$$

where $\mathbb{C}_{\text {III }+ \text { IV }}$ is the lower-half plane. The two jumps on $\operatorname{Im} k=0$ and the jump on $\operatorname{Re} k=0$ (with $\operatorname{Im} k \geq 0$ ) then define a scalar RHP:

$$
\begin{array}{lrl}
\mu^{(1)}(x, t, k)-\mu^{(3)}(x, t, k) & =\mathrm{e}^{i k x-i k^{2} t} \hat{F}(k, T) & \operatorname{Re} k=0 \wedge \operatorname{Im} k \geq 0, \\
& \\
\mu^{(1)}(x, t, k)-\mu^{(2)}(x, t, k)=\mathrm{e}^{i k x-i k^{2} t} \hat{q}(k, 0), & \operatorname{Im} k=0 \wedge \operatorname{Re} k \leq 0, \\
\mu^{(3)}(x, t, k)-\mu^{(2)}(x, t, k)=\mathrm{e}^{i k x-i k^{2} t}(\hat{q}(k, 0)-\hat{F}(k, T)), & \operatorname{Im} k=0 \wedge \operatorname{Re} k \geq 0,
\end{array}
$$


where $\hat{F}(k, t)=i \hat{f}_{1}(k, t)-k \hat{f}_{0}(k, t)$, and with

$$
\hat{q}(k, t)=\int_{0}^{\infty} \mathrm{e}^{-i k x} q(x, t) \mathrm{d} x, \quad \hat{f}_{n}(k, t)=\left.\int_{0}^{t} \mathrm{e}^{i k^{2} t^{\prime}} \partial_{x}^{n} q\left(x, t^{\prime}\right)\right|_{x=0} \mathrm{~d} t^{\prime} .
$$

The one-sided Fourier transform $\hat{q}(k, t)$ is analytic and bounded for $\operatorname{Im} k<0$, while the transforms $\hat{f}_{n}(k, t)$ of the boundary data are entire, and are bounded for $\operatorname{Im} k^{2} \geq 0$. Moreover, $\hat{q}(k, t) \rightarrow 0$ as $k \rightarrow \infty$ with $\operatorname{Im} k<0$, and $\hat{f}_{n}(z, t) \rightarrow 0$ as $k \rightarrow \infty$ with $\operatorname{Im} k^{2}<0$. The solution of the RHP defined by (5.14) is thus given by

$$
\mu(x, t, k)=\frac{1}{2 \pi i} \int_{-\infty}^{\infty} \mathrm{e}^{i k^{\prime} x-i k^{\prime 2} t} \frac{\hat{q}\left(k^{\prime}, 0\right)}{k^{\prime}-k} \mathrm{~d} k^{\prime}-\frac{1}{2 \pi i} \int_{\partial \mathbb{C}_{\mathrm{I}}} \mathrm{e}^{i k^{\prime} x-i k^{\prime 2} t} \frac{F\left(k^{\prime}, T\right)}{k^{\prime}-k} \mathrm{~d} k^{\prime} .
$$

Inserting (5.16) into the first of (5.7) then yields the reconstruction formula:

$$
q(x, t)=\frac{1}{2 \pi} \int_{-\infty}^{\infty} \mathrm{e}^{i k x-i k^{2} t} \hat{q}(k, 0) \mathrm{d} k-\frac{1}{2 \pi} \int_{\partial \mathbb{C}_{\mathrm{I}}} \mathrm{e}^{i k x-i k^{2} t} F(k, T) \mathrm{d} k .
$$

As in the discrete case, (5.17) still depends on the unknown boundary datum $q_{x}(0, t)$ via its transform in $F(k, t)$. Integrating (5.7) from $(0,0)$ to $(0, T),(\infty, T),(\infty, 0)$ and back yields the global relation as

$$
\int_{0}^{T} \mathrm{e}^{i k^{2} t}\left(i q_{x}(0, t)-k q(0, t)\right) \mathrm{d} t+\mathrm{e}^{i k^{2} T} \int_{0}^{\infty} \mathrm{e}^{-i k x} q(x, T) \mathrm{d} x=\int_{0}^{\infty} \mathrm{e}^{-i k x} q(x, 0) \mathrm{d} x,
$$

which holds for $\operatorname{Im} k \leq 0 \wedge \operatorname{Im} k^{2} \leq 0$, i.e., $k \in \mathbb{C}_{\mathrm{III}}$. In terms of the spectral data:

$$
i \hat{f}_{1}(k, T)-k \hat{f}_{0}(k, T)+\mathrm{e}^{i k^{2} T} \hat{q}(k, T)=\hat{q}(k, 0), \quad \forall k \in \overline{\mathbb{C}}_{\mathrm{III}} .
$$

Using the the transformation $k \rightarrow-k$, which leaves $\hat{f}_{n}(k, t)$ invariant, from (5.18a) we obtain

$$
i \hat{f}_{1}(k, T)+k \hat{f}_{0}(k, T)+\mathrm{e}^{i k^{2} T} \hat{q}(-k, T)=\hat{q}(-k, 0) \quad \forall k \in \overline{\mathbb{C}}_{\mathrm{I}} .
$$

We then solve for $\hat{f}_{1}(k, T)$ and insert the result in (5.17). [The first term in the RHS of (5.18b) yields a zero contribution to the solution.] Thus, the solution of the IBVP is given by

$$
q(x, t)=\frac{1}{2 \pi} \int_{-\infty}^{\infty} \mathrm{e}^{i k x-i k^{2} t} \hat{q}(k, 0) \mathrm{d} k-\frac{1}{2 \pi} \int_{\partial \mathbb{C}_{\mathrm{I}}} \mathrm{e}^{i k x-i k^{2} t}\left[\hat{q}(-k, 0)-2 k \hat{f}_{0}(k, T)\right] \mathrm{d} k .
$$

Note that one can replace $\hat{f}_{0}(k, T)$ with $\hat{f}_{0}(k, t)$. Also, the second integrand in (5.19) is analytic and bounded for $\operatorname{Im} k \geq 0 \wedge \operatorname{Im} k^{2} \leq 0$. Thus, one can deform the integration contour on the second integral onto the real $k$-axis and recover the sine transform solution (5.5). Unlike sine/cosine transform approaches, however, the present method can be applied to solve IBVPs with more complicated BCs, as we show next.

Robin BCs. Consider the IBVP for LS equation with Robin BCs:

$$
\alpha q(0, t)+q_{x}(0, t)=h(t),
$$

with $h(t)$ given and where $\alpha \in \mathbb{C}$ is a nonzero but otherwise arbitrary constant. In a similar way as shown in Appendix $\mathrm{C}$ for the discrete case, one obtains [23, 25]

$$
\hat{F}(k, t)=\frac{\hat{G}(k, t)}{k-i \alpha}+\frac{k+i \alpha}{k-i \alpha} \mathrm{e}^{i k^{2} t} \hat{q}(-k, t),
$$

where

$$
\hat{G}(k, t)=2 i k \hat{h}(k, t)-(k+i \alpha) \hat{q}(-k, 0)
$$


contains the known portion of (5.21a) and where $\hat{h}(k, t)$ is defined according to (5.15). Then, again following similar steps as in the discrete case, one obtains the solution of the IBVP as:

$q(x, t)=\frac{1}{2 \pi} \int_{-\infty}^{\infty} \mathrm{e}^{i k x-i k^{2} t} \hat{q}(k, 0) \mathrm{d} k-\frac{1}{2 \pi} \int_{\partial \mathbb{C}_{\mathrm{I}}} \mathrm{e}^{i k x-i k^{2} t} \frac{\hat{G}(k, t)}{k-i \alpha} \mathrm{d} k+i v_{\alpha} \mathrm{e}^{-\alpha x+i \alpha^{2} t} \hat{G}(i \alpha, t)$,

where $v_{\alpha}=1$ for $-\pi / 2<\arg \alpha<0, v_{\alpha}=1 / 2$ for $\arg \alpha=0,-\pi / 2$ and $v_{\alpha}=0$ for $0<\arg \alpha<3 \pi / 2$, and where the integral along $\partial \mathbb{C}_{\mathrm{I}}$ is to be taken in the principal value sense when $\arg \alpha=$ $0,-\pi / 2$. (The last term in the RHS of (5.22) is missing in Refs. [23, 25]. One can easily show, however, that without this term $q(x, t)$ does not satisfy the $\mathrm{BC}$ at $x=0$.)

\subsection{Nonlinear Schrödinger equation: initial value problem}

As in the linear case we assume that $q(x, 0) \in \mathcal{S}(\mathbb{R})$. Recall that the Lax pair for the NLS equation (5.1) is given by (A.2) with $p(x, t)=v q^{*}(x, t)$. For the present purposes, we consider $\Phi(x, t, k)$ to be a $2 \times 2$ matrix.

Analyticity. Introduce a modified eigenfunction which has a well-defined limit as $x \rightarrow \pm \infty$ :

$$
\mu(x, t, k)=\Phi(x, t, k) \mathrm{e}^{-i \theta(x, t, k) \sigma_{3}},
$$

with $\theta(x, t, k)=k x-2 k^{2} t$. Note $\mu(x, t, k)$ satisfies the following modified Lax pair:

$$
\mu_{x}-i k\left[\sigma_{3}, \mu\right]=\mathrm{Q} \mu, \quad \mu_{t}+2 i k^{2}\left[\sigma_{3}, \mu\right]=\mathrm{H} \mu .
$$

Then, letting $\mu(x, t, k)=\mathrm{e}^{i \theta \hat{\sigma}_{3}} \Psi(x, t, k)$, we obtain the simplified Lax pair: $\Psi_{x}=\mathrm{e}^{-i \theta \hat{\sigma}_{3}}(\mathrm{Q}) \Psi$ and $\Psi_{t}=\mathrm{e}^{-i \theta \hat{\sigma}_{3}}(\mathrm{H}) \Psi$. We then define the Jost eigenfunctions as the solutions of (5.24) that reduce to the identity as $x \rightarrow \pm \infty$ :

$$
\begin{aligned}
& \mu^{(1)}(x, t, k)=\mathrm{I}+\int_{-\infty}^{x} \mathrm{e}^{i k\left(x-x^{\prime}\right) \hat{\sigma}_{3}}\left(\mathrm{Q}\left(x^{\prime}, t\right) \mu^{(1)}\left(x^{\prime}, t, k\right)\right) \mathrm{d} x^{\prime}, \\
& \mu^{(2)}(x, t, k)=\mathrm{I}-\int_{x}^{\infty} \mathrm{e}^{i k\left(x-x^{\prime}\right) \hat{\sigma}_{3}}\left(\mathrm{Q}\left(x^{\prime}, t\right) \mu^{(2)}\left(x^{\prime}, t, k\right)\right) \mathrm{d} x^{\prime} .
\end{aligned}
$$

We have the following regions of analyticity and boundedness [6]:

$$
\mu^{(1, L)}, \mu^{(2, R)}: \quad \operatorname{Im} k<0, \quad \mu^{(1, R)}, \mu^{(2, L)}: \quad \operatorname{Im} k>0,
$$

where $\mu^{(j)}(x, t, k)=\left(\mu^{(j, L)}, \mu^{(j, R)}\right)$, as before. The analyticity properties of $\Phi^{(j)}(x, t, k)=$ $\mu^{(j)}(x, t, k) \mathrm{e}^{i \theta \sigma_{3}}, j=1,2$, follow trivially.

Scattering matrix. Note $\operatorname{det} \Phi^{(j)}=\operatorname{det} \mu^{(j)}=1$ for $j=1,2$. Thus $\Phi^{(1)}$ and $\Phi^{(2)}$ are both fundamental solutions of (A.2) $\forall k \in \mathbb{R}$. Hence $\Phi^{(1)}(x, t, k)=\Phi^{(2)}(x, t, k) \mathrm{A}(k)$, where $\mathrm{A}(k)$ is the scattering matrix. Equivalently,

$$
\mu^{(1)}(x, t, k)=\mu^{(2)}(x, t, k) \mathrm{e}^{i \theta \hat{\sigma}_{3}} \mathrm{~A}(k) .
$$

Note that $\mathrm{A}(k)$ is indeed independent of time, and $\operatorname{det} \mathrm{A}(k)=1$. Moreover,

$$
\mathrm{A}(k)=\mathrm{I}+\int_{-\infty}^{\infty} \mathrm{e}^{-i\left(k x-2 k^{2} t\right) \hat{\sigma}_{3}}\left(\mathrm{Q}(x, t) \mu^{(1)}(x, t, k)\right) \mathrm{d} x,
$$

and

$$
\begin{aligned}
& a_{11}(k)=\operatorname{Wr}\left(\Phi^{(1, L)}, \Phi^{(2, R)}\right), \quad a_{12}(k)=\operatorname{Wr}\left(\Phi^{(1, R)}, \Phi^{(2, R)}\right), \\
& a_{21}(k)=-\operatorname{Wr}\left(\Phi^{(1, L)}, \Phi^{(2, L)}\right), \quad a_{22}(k)=-\operatorname{Wr}\left(\Phi^{(1, R)}, \Phi^{(2, L)}\right) .
\end{aligned}
$$

Thus, $a_{11}(k)$ and $a_{22}(k)$ can be analytically continued respectively on $\operatorname{Im} k<0$ and $\operatorname{Im} k>0$, but $a_{12}(k)$ and $a_{21}(k)$ are nowhere analytic, in general. 
Symmetries. When $p(x, t)=v q^{*}(x, t)$, with $v= \pm 1$, the scattering problem (A.2) admits an involution expressed via the matrix $\sigma_{v}$ in (A.11a): if $\Phi(x, t, k)$ is a solution of (A.2a), so is

$$
\Phi^{\prime}(x, t, k)=\sigma_{v} \Phi^{*}\left(x, t, k^{*}\right) .
$$

Comparing the behavior of the Jost eigenfunctions as $x \rightarrow \pm \infty$ we then have

$$
\Phi^{(j, L)}(x, t, k)=\sigma_{v}\left(\Phi^{(j, R)}\left(x, t, k^{*}\right)\right)^{*}, \quad \Phi^{(j, R)}(x, t, k)=v \sigma_{v}\left(\Phi^{(j, L)}\left(x, t, k^{*}\right)\right)^{*},
$$

for $j=1,2$. Hence the following relations hold for the elements of the scattering matrix $\mathrm{A}(k)$ :

$$
a_{22}(k)=a_{11}^{*}\left(k^{*}\right), \quad a_{21}(k)=v a_{12}^{*}\left(k^{*}\right) .
$$

Note that, since $\operatorname{det} \mathrm{A}(k)=1$, (5.31) imply $\left|a_{11}(k)\right|^{2}-v\left|a_{12}(k)\right|^{2}=1 \forall k \in \mathbb{R}$.

Asymptotics. The asymptotics of the Jost solutions as $k \rightarrow \infty$ in their half planes is:

$$
\begin{aligned}
& \mu^{(1)}(x, t, k)=\mathrm{I}-\frac{1}{2 i k} \sigma_{3} \mathrm{Q}+\frac{1}{2 i k} \sigma_{3} \int_{-\infty}^{x} q\left(x^{\prime}, t\right) p\left(x^{\prime}, t\right) \mathrm{d} x^{\prime}+O\left(1 / k^{2}\right), \\
& \mu^{(2)}(x, t, k)=\mathrm{I}-\frac{1}{2 i k} \sigma_{3} \mathrm{Q}-\frac{1}{2 i k} \sigma_{3} \int_{x}^{\infty} q\left(x^{\prime}, t\right) p\left(x^{\prime}, t\right) \mathrm{d} x^{\prime}+O\left(1 / k^{2}\right) .
\end{aligned}
$$

Moreover, from (5.28) and (5.32) one also obtains

$$
a_{22}(k)=1-\frac{1}{2 i k} \int_{-\infty}^{\infty} q(x, t) p(x, t) \mathrm{d} x+O\left(1 / k^{2}\right) .
$$

Inverse problem. The inverse problem is the RHP defined by (5.26) for $k \in \mathbb{R}$ :

$$
\mathrm{M}^{-}(x, t, k)=\mathrm{M}^{+}(x, t, k)(\mathrm{I}-\mathrm{J}(k, t)),
$$

where the matrix-valued sectionally meromorphic functions are

$$
\mathrm{M}^{+}(x, t, k)=\left(\mu^{(2, L)}(x, t, k), \frac{\mu^{(1, R)}(x, t, k)}{a_{22}(k)}\right), \quad \mathrm{M}^{-}(x, t, k)=\left(\frac{\mu^{(1, L)}(x, t, k)}{a_{11}(k)}, \mu^{(2, R)}(x, t, k)\right),
$$

the jump matrix is

$$
\mathrm{J}(k, t)=\left(\begin{array}{cc}
\rho_{1}(k) \rho_{2}(k) & \mathrm{e}^{2 i \theta} \rho_{2}(k) \\
-\mathrm{e}^{-2 i \theta} \rho_{1}(k) & 0
\end{array}\right),
$$

and the reflection coefficients, defined $\forall k \in \mathbb{R}$, are

$$
\rho_{1}(k)=a_{21}(k) / a_{11}(k), \quad \rho_{2}(k)=a_{12}(k) / a_{22}(k) .
$$

Of course (5.31) imply $\rho_{1}(k)=v \rho_{2}^{*}\left(k^{*}\right)$ when $p(x, t)=v q^{*}(x, t)$. In the absence of a discrete spectrum [i.e., if $a_{11}(k) \neq 0 \forall \operatorname{Im} k<0$ and $a_{22}(k) \neq 0 \forall \operatorname{Im} k>0$ ] the matrix functions $\mathrm{M}^{ \pm}(x, t, k)-\mathrm{I}$ are sectionally analytic in their respective half planes, and they vanish as $k \rightarrow \infty$. Therefore the RHP (5.34) is solved via the Cauchy projectors $P^{ \pm}$, as for the linear case:

$$
\mathrm{M}^{+}(x, t, k)=\mathrm{I}+\frac{1}{2 \pi i} \int_{-\infty}^{\infty} \mathrm{M}^{+}\left(x, t, k^{\prime}\right) \frac{\mathrm{J}\left(k^{\prime}, t\right)}{k^{\prime}-k} \mathrm{~d} k^{\prime} .
$$

The asymptotic behavior of $\mathrm{M}(x, t, k)$ as $k \rightarrow \infty$ is easily obtained from (5.35): for $\operatorname{Im} k>0$,

$$
\mathrm{M}^{+}(x, t, k)=\mathrm{I}-\frac{1}{2 i \pi k} \int_{-\infty}^{\infty} \mathrm{M}^{+}\left(x, t, k^{\prime}\right) \mathrm{J}\left(k^{\prime}, t\right) \mathrm{d} k^{\prime}+O\left(1 / k^{2}\right) .
$$

Comparing the (1,2)-components of (5.36) and (5.32) then yields the reconstruction formula:

$$
q(x, t)=\frac{1}{\pi} \int_{-\infty}^{\infty} \mathrm{e}^{2 i\left(k x-2 k^{2} t\right)} \rho_{2}(k)\left(\mu^{(2)}(x, t, k)\right)_{11} \mathrm{~d} k .
$$


Linear limit. If $\mathrm{Q}(x, t)=O(\varepsilon)$ one has $\mu(x, t, k)=\mathrm{I}+O(\varepsilon)$ and, to $O(\varepsilon)$,

$$
\mathrm{A}(k)=\mathrm{I}+\int_{-\infty}^{\infty} \mathrm{e}^{-i\left(k x-2 k^{2} t\right) \hat{\sigma}_{3}} \mathrm{Q}(x, t) \mathrm{d} x
$$

From here and (5.37) one then obtains, to $O(\varepsilon)$,

$$
q(x, t)=\frac{1}{\pi} \int_{-\infty}^{\infty} e^{2 i\left(k x-2 k^{2} t\right)} \rho_{2}(k) \mathrm{d} k, \quad \rho_{2}(k)=\int_{-\infty}^{\infty} \mathrm{e}^{-2 i k x} q(x, 0) \mathrm{d} x,
$$

which, with the familiar rescaling $k^{\prime}=2 k$, coincide with the Fourier transform pair (5.2).

\subsection{Nonlinear Schrödinger equation: initial-boundary value problem}

We now discuss the IBVP for the NLS equation (5.1) on the half line. As in the linear case, we assume $q(x, 0) \in \mathcal{S}\left(\mathbb{R}^{+}\right)$and $q(0, t) \in C\left(\mathbb{R}^{+}\right)$.

Eigenfunctions and analyticity. Introduce three Jost eigenfunctions as the solutions of (5.24) that reduce to the identity respectively at $(x, t)=(0,0),(x, t) \rightarrow(\infty, t)$ and $(x, t)=(0, T)$ :

$$
\begin{aligned}
& \mu^{(1)}(x, t, k)=\mathrm{I}+\int_{0}^{x} \mathrm{e}^{i k\left(x-x^{\prime}\right) \hat{\sigma}_{3}}\left(\mathrm{Q}\left(x^{\prime}, t\right) \mu^{(1)}\left(x^{\prime}, t, k\right)\right) \mathrm{d} x^{\prime} \\
& +\int_{0}^{t} \mathrm{e}^{i\left[k x-2 k^{2}\left(t-t^{\prime}\right)\right] \hat{\sigma}_{3}}\left(\mathrm{H}\left(0, t^{\prime}, k\right) \mu^{(1)}\left(0, t^{\prime}, k\right)\right) \mathrm{d} t^{\prime}, \\
& \mu^{(2)}(x, t, k)=\mathrm{I}-\int_{x}^{\infty} \mathrm{e}^{i k\left(x-x^{\prime}\right) \hat{\sigma}_{3}}\left(\mathrm{Q}\left(x^{\prime}, t\right) \mu^{(2)}\left(x^{\prime}, t, k\right)\right) \mathrm{d} x^{\prime}, \\
& \mu^{(3)}(x, t, k)=\mathrm{I}+\int_{0}^{x} \mathrm{e}^{i k\left(x-x^{\prime}\right) \hat{\sigma}_{3}}\left(\mathrm{Q}\left(x^{\prime}, t\right) \mu^{(3)}\left(x^{\prime}, t, k\right)\right) \mathrm{d} x^{\prime} \\
& -\int_{t}^{T} \mathrm{e}^{i\left[k x-2 k^{2}\left(t-t^{\prime}\right)\right] \hat{\sigma}_{3}}\left(\mathrm{H}\left(0, t^{\prime}, k\right) \mu^{(3)}\left(0, t^{\prime}, k\right)\right) \mathrm{d} t^{\prime} .
\end{aligned}
$$

Note that $\mu^{(1)}(x, t, k)$ and $\mu^{(3)}(x, t, k)$ are entire functions of $k$, while $\mu^{(2)}(x, t, k)$ coincides with (5.25b). Moreover, (5.38) imply the following domains of analyticity and boundedness:

$$
\begin{array}{llll}
\mu^{(1, L)}: & \mathbb{C}_{\mathrm{III}}, & \mu^{(1, R)}: \mathbb{C}_{\mathrm{II}}, \quad \mu^{(3, L)}: \mathbb{C}_{\mathrm{IV}}, \quad \mu^{(3, R)}: \mathbb{C}_{\mathrm{I}}, \\
\mu^{(2, L)}: & \mathbb{C}_{\mathrm{I}+\mathrm{II}}, \quad \mu^{(2, R)}: \mathbb{C}_{\mathrm{III}+\mathrm{IV} .} &
\end{array}
$$

Scattering matrices. We still have $\operatorname{det} \Phi^{(j)}(x, t, k)=1$ for all $x, t \in \mathbb{R}^{+}$and for all $j=1,2,3$. Hence the matrices $\Phi^{(j)}(x, t, k), j=1,2,3$ are three fundamental solutions of the Lax pair (A.2), and they must be proportional to each other. In terms of the modified eigenfunctions:

$$
\begin{aligned}
& \mu^{(2)}(x, t, k)=\mu^{(1)}(x, t, k) \mathrm{e}^{i\left(k x-2 k^{2} t\right) \hat{\sigma}_{3}} \mathrm{~S}(k), \\
& \mu^{(3)}(x, t, k)=\mu^{(1)}(x, t, k) \mathrm{e}^{i\left(k x-2 k^{2} t\right) \hat{\sigma}_{3}} \mathrm{~S}(k, T) .
\end{aligned}
$$

Note that the first column of $(5.39 a)$ is defined $\forall k \in \overline{\mathbb{C}}_{\mathrm{I}+\mathrm{II}}$, the second column $\forall k \in \overline{\mathbb{C}}_{\mathrm{III}+\mathrm{IV}}$ and $(5.39 b)$ holds $\forall k \in \mathbb{C}$. Also, $\operatorname{det} \mathrm{s}(k)=\operatorname{det} \mathrm{S}(k, T)=1$. The scattering matrices $\mathrm{s}(k)$ and $\mathrm{S}(k, T)$ are obtained from the boundary values of the eigenfunctions, namely, $\forall k \in \mathbb{C}$,

$$
\mathrm{s}(k)=\mu^{(2)}(0,0, k), \quad \mathrm{S}(k, T)=\left(\mathrm{e}^{2 i k^{2} T \hat{\sigma}_{3}} \mu^{(1)}(0, T, k)\right)^{-1} .
$$


Then, from (5.38) we have the following integral representations of the scattering matrices:

$$
\begin{aligned}
& \mathrm{S}(k)=\mathrm{I}-\int_{0}^{\infty} \mathrm{e}^{-i k x \hat{\sigma}_{3}}\left(\mathrm{Q}(x, 0) \mu^{(2)}(x, 0, k)\right) \mathrm{d} x, \\
& \mathrm{~S}^{-1}(k, T)=\mathrm{I}+\int_{0}^{T} \mathrm{e}^{2 i k^{2} t \hat{\sigma}_{3}}\left(\mathrm{H}(0, t, k) \mu^{(1)}(0, t, k)\right) \mathrm{d} t .
\end{aligned}
$$

These imply that: $\mathbf{s}_{L}(k)$ and $\mathbf{s}_{R}(k)$ are analytic respectively for $k \in \mathbb{C}_{\mathrm{I}+\mathrm{II}}$ and $k \in \mathbb{C}_{\mathrm{III}+\mathrm{IV}}$, and their restriction to these domains are continuous and bounded on the boundary; $\mathrm{S}(k, T)$ is entire, and $\mathrm{S}_{L}(k, T)$ and $\mathrm{S}_{R}(k, T)$ are bounded respectively for $k \in \overline{\mathbb{C}}_{\mathrm{II}+\mathrm{IV}}$ and $k \in \overline{\mathbb{C}}_{\mathrm{I}+\mathrm{III}}$.

Symmetries, discrete spectrum and asymptotics. When $p(x, t)=v q^{*}(x, t),(5.29)$ still holds, as does (5.30) for $j=1,2,3$. This implies that the scattering matrices can be expressed as

$$
\mathrm{s}(k)=\left(\begin{array}{cc}
a(k) & v b^{*}\left(k^{*}\right) \\
b(k) & a^{*}\left(k^{*}\right)
\end{array}\right), \quad \mathrm{S}(k, T)=\left(\begin{array}{cc}
A(k, T) & v B^{*}\left(k^{*}, T\right) \\
B(k, T) & A^{*}\left(k^{*}, T\right)
\end{array}\right) .
$$

The properties of $a(k), b(k), A(k, T)$ and $B(k, T)$ follow trivially from those of $\mathrm{s}(k)$ and $\mathrm{S}(k, T)$. Also, one can show that $\mu^{(j)}(x, t, k)=\mathrm{I}+O(1 / k)$ for $j=1,2,3$ as $k \rightarrow \infty$ in the respective domains of boundedness of their columns. The asymptotics of the eigenfunctions then determines that of the scattering matrices. In particular, $a(k)=1+O(1 / k)$ and $b(k)=O(1 / k)$ as $k \rightarrow \infty$ in $\overline{\mathbb{C}}_{\mathrm{I}+\mathrm{II}}$, and $A(k, T)=1+O(1 / k)$ and $B(k, T)=O(1 / k)$ as $k \rightarrow \infty$ in $\overline{\mathbb{C}}_{\mathrm{III}+\mathrm{IV}}$.

Riemann-Hilbert problem, solution and reconstruction formula. Equations (5.39) allow us to formulate the following RHP:

$$
\mathrm{M}^{-}(x, t, k)=\mathrm{M}^{+}(x, t, k)(\mathrm{I}-\mathrm{J}(k, t)), \quad k \in L,
$$

with $L=\partial \mathbb{C}_{\mathrm{I}} \cup \partial \mathbb{C}_{\text {III }}=L_{1} \cup L_{2} \cup L_{3} \cup L_{4}$, where

$$
L_{1}=\overline{\mathbb{C}}_{\mathrm{I}} \cap \overline{\mathbb{C}}_{\mathrm{II}}, \quad L_{2}=\overline{\mathbb{C}}_{\mathrm{II}} \cap \overline{\mathbb{C}}_{\mathrm{III}}, \quad L_{3}=\overline{\mathbb{C}}_{\mathrm{III}} \cap \overline{\mathbb{C}}_{\mathrm{IV}}, \quad L_{4}=\overline{\mathbb{C}}_{\mathrm{I}} \cap \overline{\mathbb{C}}_{\mathrm{IV}},
$$

and where

$$
\mathrm{M}^{+}(x, t, k)=\left\{\begin{array}{ll}
\left(\mu^{(2, L)}, \frac{\mu^{(3, R)}}{d(k)}\right), & k \in \mathbb{C}_{\mathrm{I}}, \\
\left(\frac{\mu^{(1, L)}}{a^{*}\left(k^{*}\right)}, \mu^{(2, R)}\right), & k \in \mathbb{C}_{\mathrm{III}},
\end{array} \quad \mathrm{M}^{-}(x, t, k)= \begin{cases}\left(\mu^{(2, L)}, \frac{\mu^{(1, R)}}{a(k)}\right), & k \in \mathbb{C}_{\mathrm{II}}, \\
\left(\frac{\mu^{(3, L)}}{d^{*}\left(k^{*}\right)}, \mu^{(2, R)}\right), & k \in \mathbb{C}_{\mathrm{IV}} .\end{cases}\right.
$$

The jump matrices $\mathrm{J}_{j}(k, t)$, each defined for $k \in L_{j}$, are:

$$
\begin{array}{ll}
\mathrm{J}_{1}(k, t)=\left(\begin{array}{cc}
0 & v \mathrm{e}^{2 i \theta} \Gamma^{*}\left(k^{*}\right) \\
0 & 0
\end{array}\right), & \mathrm{J}_{2}(k, t)=\left(\begin{array}{cc}
0 & \mathrm{e}^{2 i \theta} \gamma(k) \\
-v \mathrm{e}^{-2 i \theta} \gamma^{*}(k) & v|\gamma(k)|^{2}
\end{array}\right), \\
\mathrm{J}_{3}(k, t)=\left(\begin{array}{cc}
0 & 0 \\
-\mathrm{e}^{-2 i \theta} \Gamma(k) & 0
\end{array}\right), & \mathrm{J}_{4}(k, t)=\mathrm{I}-\left(\mathrm{I}-\mathrm{J}_{1}\right)\left(\mathrm{I}-\mathrm{J}_{2}\right)^{-1}\left(\mathrm{I}-\mathrm{J}_{3}\right),
\end{array}
$$

and the reflection coefficients are

$\gamma(k)=\frac{v b^{*}(k)}{a(k)}, \quad d(k)=a(k) A^{*}\left(k^{*}, T\right)-v b(k) B^{*}\left(k^{*}, T\right), \quad \Gamma(k)=\frac{B(k, T)}{a^{*}\left(k^{*}\right) d^{*}\left(k^{*}\right)}$.

Note that $d(k)$ is defined $\forall k \in \overline{\mathbb{C}}_{\mathrm{I}+\mathrm{II}}, \Gamma(k)$ for $k \in L_{3} \cup L_{4}$ and $\gamma(k) \forall k \in \mathbb{R}$. Their asymptotics as $k \rightarrow \infty$ follow trivially from those of $\mathbf{S}(k)$ and $\mathrm{S}(k, T)$. As a result, $\mathrm{M}(x, t, k) \rightarrow \mathrm{I}$ as $k \rightarrow \infty$. Hence, in the absence of a discrete spectrum [that is, assuming that $a(k)$ and $d(k)$ have no zero respectively for $k \in \mathbb{C}_{\mathrm{II}}$ and $k \in \mathbb{C}_{\mathrm{I}}$ ], the RHP (5.42) is solved by Cauchy projectors:

$$
\mathrm{M}^{+}(x, t, k)=\mathrm{I}+\frac{1}{2 \pi i} \int_{L} \mathrm{M}^{+}\left(x, t, k^{\prime}\right) \frac{\mathrm{J}\left(k^{\prime}, t\right)}{k^{\prime}-k} \mathrm{~d} k^{\prime} .
$$


Substituting the asymptotic expansion for $\mathrm{M}(x, t, k)$ into the $x$-part of the Lax pair and comparing the $(1,2)$ components, we have

$$
q(x, t)=-2 i \lim _{k \rightarrow \infty} k(\mathrm{M}(x, t, k)-\mathrm{I})_{12} .
$$

Using the asymptotic expansion for $\mathrm{M}(x, t, k)$ as $k \rightarrow \infty$, from (5.43) and comparing the $(1,2)$ components, we obtain the solution of the IBVP for the NLS equation as

$$
\begin{aligned}
q(x, t)=\frac{1}{\pi} \int_{\partial \mathbb{C}_{\mathrm{I}}} v \mathrm{e}^{2 i \theta\left(x, t, k^{\prime}\right)} \Gamma^{*}\left(k^{\prime *}\right) \mathrm{M}_{11}^{+}\left(x, t, k^{\prime}\right) \mathrm{d} k^{\prime} \\
\quad-\frac{1}{\pi} \int_{-\infty}^{\infty} \mathrm{e}^{2 i \theta\left(x, t, k^{\prime}\right)} \gamma\left(k^{\prime}\right) \mathrm{M}_{11}^{+}\left(x, t, k^{\prime}\right) \mathrm{d} k^{\prime}-\frac{1}{\pi} \int_{0}^{\infty} v\left|\gamma\left(-k^{\prime}\right)\right|^{2} \mathrm{M}_{12}^{+}\left(x, t,-k^{\prime}\right) \mathrm{d} k^{\prime} .
\end{aligned}
$$

Linear limit. Supppose that $\mathrm{Q}=O(\varepsilon)$. From (5.38) and (5.45) we have $\mu=\mathrm{I}+O(\varepsilon)$ and $\mathrm{M}=\mathrm{I}+O(\varepsilon)$. Also, (5.41) imply $\gamma(k)=-\hat{q}(2 k, 0)+O\left(\varepsilon^{2}\right)$ and $d(k)=1+O\left(\varepsilon^{2}\right)$, as well as

$$
\Gamma^{*}\left(k^{*}\right)=v\left(2 k \hat{f}_{0}(2 k, T)-i \hat{f}(2 k, T)\right)+O\left(\varepsilon^{2}\right) .
$$

Thus (5.45) yields, to $O(\varepsilon)$,

$q(x, t)=\frac{1}{\pi} \int_{\partial \mathbb{C}_{\mathrm{I}}} \mathrm{e}^{2 i\left(k^{\prime} x-2 k^{\prime 2} t\right)}\left(2 k^{\prime} \hat{f}_{0}\left(2 k^{\prime}, T\right)-i \hat{f}_{1}\left(2 k^{\prime}, T\right)\right) \mathrm{d} k^{\prime}+\frac{1}{\pi} \int_{-\infty}^{\infty} \mathrm{e}^{2 i\left(k^{\prime} x-2 k^{\prime 2} t\right)} \hat{q}\left(2 k^{\prime}, 0\right) \mathrm{d} k^{\prime}$.

Performing the change of variable $2 k^{\prime}=k$, we then see that, to leading order, this expression yields exactly the solution of the linear Schrödinger equation on the half line, namely (5.17).

Global relation and Dirichlet-to-Neumann map. Equations (5.41) involve all initial and boundary data for $\mathrm{Q}(x, t)$. These values are not all independent, however, since they satisfy the global relation

$$
\begin{aligned}
\int_{0}^{T} \mathrm{e}^{2 i k^{2} t \hat{\sigma}_{3}}(\mathrm{H}(0, t, k) \mu(0, t, k)) \mathrm{d} t+\mathrm{e}^{2 i k^{2} T \hat{\sigma}_{3}} \int_{0}^{\infty} \mathrm{e}^{-i k x \hat{\sigma}_{3}}(\mathrm{Q}(x, T) \mu(x, T, k)) \mathrm{d} x & \\
= & \int_{0}^{\infty} \mathrm{e}^{-i k x \hat{\sigma}_{3}}(\mathrm{Q}(x, 0) \mu(x, 0, k)) \mathrm{d} x .
\end{aligned}
$$

When (5.46) is evaluated with $\mu \equiv \mu^{(2)}(x, t, k)$, its first column is defined $\forall k \in \overline{\mathbb{C}}_{\mathrm{I}+\mathrm{II}}$, its second column $\forall k \in \overline{\mathbb{C}}_{\mathrm{III}+\mathrm{IV}}$. Moreover, when $\mu(x, t, k)=\mu^{(2)}(x, t, k)$, the RHS of (5.46) equals I- $\mathrm{s}(k)$. Using $(5.39 b)$ in the LHS, one then obtains a relation between the scattering matrices:

$$
\mathrm{S}^{-1}(k, T) \mathrm{S}(k)=\mathrm{I}-\mathrm{e}^{2 i k^{2} T \hat{\sigma}_{3}} \mathrm{G}(k, T),
$$

where

$$
\mathrm{G}(k, t)=\int_{0}^{\infty} \mathrm{e}^{-i k x \hat{\sigma}_{3}}\left(\mathrm{Q}(x, t) \mu^{(2)}(x, t, k)\right) \mathrm{d} x,
$$

and $\mathrm{G}_{L}(k, t)$ and $\mathrm{G}_{R}(k, t)$ are analytic respectively for $k \in \mathbb{C}_{\mathrm{I}+\mathrm{II}}$ and $k \in \mathbb{C}_{\mathrm{III}+\mathrm{IV}}$, and continuous and bounded on the boundary of these domains. In particular, for $k \in \mathbb{C}_{\text {III+IV }}$ we have

$$
A^{*}\left(k^{*}, T\right) b^{*}\left(k^{*}\right)-B^{*}\left(k^{*}, T\right) a^{*}(k)=-v \mathrm{e}^{4 i k^{2} T} \int_{0}^{\infty} \mathrm{e}^{-2 i k x} q(x, T) \mu_{22}^{(2)}(x, T, k) \mathrm{d} x .
$$

Since the integral term in the RHS is of $O(1 / k)$, as $k \rightarrow \infty$ in $\mathbb{C}_{\text {III }}$, integrating along $\partial \mathbb{C}_{\text {III }}$ we obtain the following integral relation:

$$
\int_{\partial \mathrm{C}_{\text {III }}} k \mathrm{e}^{-4 i k^{2} t}\left(B^{*}\left(k^{*}\right)-r\left(k^{*}\right) A^{*}\left(k^{*}\right)\right) \mathrm{d} k=0,
$$

where $r(k)=b(k) / a(k)$. As shown in [28], this relation can be solved to obtain the Dirichlet-toNeumann map, which expresses the unknown boundary datum $q_{x}(0, t)$ in terms of the known one, $q(0, t)$. 
Linearizable BCs and soliton solutions. One can write $\mathrm{S}(k, t)=\tilde{\Phi}^{-1}(k, t) \mathrm{e}^{-2 i k^{2} t \sigma_{3} \hat{\sigma}_{3}}$, where $\tilde{\Phi}(k, t)=\Phi^{(1)}(0, t, k)$ solves the $t$-part of the Lax pair (A.2b) for $x=0$, namely

$$
\tilde{\Phi}_{t}+2 i k^{2} \sigma_{3} \tilde{\Phi}=\mathrm{H}(0, t, k) \tilde{\Phi}
$$

with $\tilde{\Phi}(k, 0)=$ I. The matrix $\tilde{\Phi}(-k, t)$ solves an equation identical to (5.49) except that $\mathrm{H}(0, t, k)$ is replaced by $\mathrm{H}(0, t,-k)$. If there is an invertible time-independent matrix $\mathrm{N}(k)$ such that

$$
\mathrm{N}(k)\left(2 i k^{2} \sigma_{3}-\mathrm{H}(0, t, k)\right)=\left(2 i k^{2} \sigma_{3}-\mathrm{H}(0, t,-k)\right) \mathrm{N}(k),
$$

it then is easy to see that $\tilde{\Phi}(-k, t)=\mathrm{N}(k) \tilde{\Phi}(k, t) \mathrm{N}^{-1}(k)$. One can show that a suitable matrix $\mathrm{N}(k)$ only exists for homogeneous Robin BCs, namely,

$$
q_{x}(0, t)-\chi q(0, t)=0 \text {, }
$$

with $\chi \in \mathbb{R}$ arbitrary. In that case, (5.50) implies $N_{12}=N_{21}=0$ and $N_{11}=f(k) N_{22}$, where $f(k)=-(2 i k-\chi) /(2 i k+\chi)$, which in turn imply $A^{*}\left(k^{*}, T\right)=A^{*}\left(-k^{*}, T\right)$ and $B^{*}\left(k^{*}, T\right)=$ $f(k) B^{*}\left(-k^{*}, T\right)$. From here, similar arguments to those used in the discrete problem can be applied to the analysis of linearizable BCs.

As in the discrete case, the poles for the IBVP occur at the zeros of $a(k)$ in $\mathbb{C}_{\mathrm{II}}$ and those of $d(k)$ in $\mathbb{C}_{\mathrm{I}}$, plus their complex conjugates in $\mathbb{C}_{\mathrm{III}}$ and $\mathbb{C}_{\mathrm{IV}}$ [29]. Each of these pairs of zeros, by itself, generates the well-known one-soliton solution of NLS:

$$
q(x, t)=2 \eta \mathrm{e}^{2 i \xi x-4 i\left(\xi^{2}-\eta^{2}\right) t+i(\phi-\pi / 2)} \operatorname{sech}(2 \eta x-8 \xi \eta t-2 \delta),
$$

where $k_{1}=\xi+i \eta$ is the zero of $a(k)$ or of $d(k)$, and $C_{1}=2 \eta \mathrm{e}^{2 \delta+i \phi}$ is the norming constant (see [29] for further details).

\section{Conclusion}

In conclusion, we have demonstrated a method to solve initial-boundary value problems for linear and integrable nonlinear discrete evolution equations. We have done so by solving the IBVP for the discrete linear Schrödinger (DLS) and integrable discrete nonlinear Schrödinger (IDNLS) equations on the natural numbers. Moreover, we have illustrated the similarities and differences between the method for differential-difference equations and PDEs by showing explicitly the correspondence between the discrete and its continuum limit. While the differential form representation of the continuum is lost, the essential ideas of the method can be carried over to the discrete, but the actual implementation of the method presents some additional difficulties. In particular, the jump location in the nonlinear case differs because of the rescaling $z^{\prime}=z^{2}$ in the dispersion relation $\omega(z)$ when going from the linear to the nonlinear case. This is a significant difference from continuum limit, where the jumps in the nonlinear case are given by the union of those for the linear problem and its adjoint (cf. sections 3 and 5). Also, the limit $k \rightarrow \infty$ in the continumm becomes $z \rightarrow 0$ (for $\operatorname{Im} k>0$ ) and $z \rightarrow \infty$ (for $\operatorname{Im} k<0)$ in the discrete. As a consequence, the behavior of the eigenfuncions and spectral data as $z \rightarrow 0$ in the discrete problem must also be studied in addition to that as $z \rightarrow \infty$. This is the why the point $z=0$ plays such a special role in the discrete problem, similarly to Ref. [1], and is one of the reason why discrete problems are more complicated than their continuum counterparts.

For the DLS, in addition to solving the IBVP with Dirichlet-type BCs we have shown that, contrary to Fourier series approaches, the method can deal with more complicated kinds of BCs just as effectively. For the IDNLS, in addition to solving the IBVP (showing explicitly how to eliminate the unknown boundary datum), we have characterized the linear limit, the linearizable BCs (showing how they fit within the IST framework), and we have obtained explicitly the soliton solutions. 
It should be clear that, similarly to the continuum, the method can be generalized to solve IBVPs for both the DLS and IDNLS equation defined on a finite set of integers. It would also be straightforward to generalize this method to any discrete linear evolution equation and to other integrable discrete nonlinear evolution equations.

Several interesting questions can now be effectively addressed using the present method. For example, one can use the expression for the solution to study its long-time asymptotics, using the Deift-Zhou method [17], or to study the "small dispersion" or "anti-continuum" limit (i.e., the limit $h \rightarrow \infty$ ), e.g., using the Deift-Venakides-Zhou method [16]. Doing so is a nontrivial task, however, which is beyond the scope of this work.

\section{Acknowledgements}

It is a pleasure to thank Mark Ablowitz, Athanassios Fokas, Beatrice Pelloni and Barbara Prinari for many insightful discussions. This work was partially supported by the National Science Foundation under grant number DMS-0506101.

\section{Appendix A. Notation and frequently used formulae}

We denote the closure, interior and boundary of a domain $D$ respectively by $\bar{D}, D^{o}$ and $\partial D$, where as usual $\partial D$ is oriented so as to leave $D$ to its left. We also occasionally refer to punctured regions of the complex plane, which we denote as $R^{[\emptyset]}=R-\{0\}$. As usual, $[A, B]=A B-B A$ is the commutator of two matrices $A$ and $B$. We use a superscript asterisk to denote the complex conjugate $z^{*}$ of a complex number $z$, and $|z|^{2}=z^{*} z$. Throughout, $\mathbb{R}^{+}=\{x \in \mathbb{R}: x>0\}$ and $\mathbb{R}_{0}^{+}=\mathbb{R}^{+} \cup\{0\}$. Similarly, $\mathbb{N}=\{1,2,3, \ldots\}$ and $\mathbb{N}_{0}=\mathbb{N} \cup\{0\}$. Finally, we denote by $\mathbb{C}_{\mathrm{I}}, \ldots, \mathbb{C}_{\mathrm{IV}}$ the first, second, third and fourth quadrants of the complex plane: $\mathbb{C}_{\mathrm{I}}=\{k \in \mathbb{C}: \operatorname{Re} k>0 \wedge \operatorname{Im} k>0\}$, etc. Similarly, we denote by $\mathbb{C}_{\mathrm{I}+\mathrm{II}}=\{k \in \mathbb{C}: \operatorname{Im} k>0\}$ and $\mathbb{C}_{\text {III }+ \text { IV }}=\{k \in \mathbb{C}: \operatorname{Im} k<0\}$ the upper-half and lower-half planes, respectively.

The nonlinear Schrödinger (NLS) equation (5.1) is a reduction of the system

$$
\begin{aligned}
& i q_{t}+q_{x x}+2 q^{2} p=0, \\
& -i p_{t}+p_{x x}+2 p^{2} q=0 .
\end{aligned}
$$

That is, (5.1) follows by imposing $p(x, 0)=v q^{*}(x, 0)$ in (A.1), which then implies that $p(x, t)=v q^{*}(x, t) \forall t>0$ and $q(x, t)$ is a solution of (5.1). A Lax pair for (A.1) is given by:

$$
\begin{aligned}
& \Phi_{x}-i k \sigma_{3} \Phi=\mathrm{Q} \Phi, \\
& \Phi_{t}+2 i k^{2} \sigma_{3} \Phi=\mathrm{H} \Phi,
\end{aligned}
$$

where $\Phi(x, t, k)$ is either a 2 -component vector or a $2 \times 2$ matrix, and where

$$
\begin{aligned}
& \sigma_{3}=\left(\begin{array}{cc}
1 & 0 \\
0 & -1
\end{array}\right), \quad \mathrm{Q}(x, t)=\left(\begin{array}{cc}
0 & q \\
p & 0
\end{array}\right), \\
& \mathrm{H}(x, t, k)=i \sigma_{3}\left(\mathrm{Q}_{x}-\mathrm{Q}^{2}\right)-2 k \mathrm{Q}=\left(\begin{array}{cc}
-i q p & i q_{x}-2 k q \\
-i p_{x}-2 k p & i q p
\end{array}\right) .
\end{aligned}
$$

(The present pair differs from that in Ref. [26] by the rescaling $k \rightarrow-k$, and from that in Ref. [6] by $k \rightarrow-k$ and $t \rightarrow-t$.) Similarly, the integrable discrete NLS equation (1.2) is a reduction of the system of differential-difference equations

$$
\begin{aligned}
& i \dot{q}_{n}+\left(q_{n+1}-2 q_{n}+q_{n-1}\right)-q_{n} p_{n}\left(q_{n+1}+q_{n-1}\right)=0, \\
& i \dot{p}_{n}+\left(p_{n+1}-2 p_{n}+p_{n-1}\right)-p_{n} q_{n}\left(p_{n+1}+p_{n-1}\right)=0 .
\end{aligned}
$$


That is, imposing $p_{n}(0)=v q_{n}^{*}(0)$ on (A.4) yields $p_{n}(t)=v q_{n}^{*}(t) \quad \forall t>0$, with $q_{n}(t)$ satisfying (1.2). In the literature, the name Ablowitz-Ladik (AL) is associated to both (1.2) and (A.4). To avoid confusion, here we will simply refer to (1.2) as the IDNLS equation, reserving the name AL for the more general system (A.4). A Lax pair for the AL system (A.4) is:

$$
\begin{aligned}
& \Phi_{n+1}-\mathrm{Z} \Phi_{n}=\mathrm{Q}_{n} \Phi_{n}, \\
& \dot{\Phi}_{n}-\frac{i}{2}(z-1 / z)^{2} \sigma_{3} \Phi_{n}=\mathrm{H}_{n} \Phi_{n},
\end{aligned}
$$

where $\Phi_{n}(z, t)$ is either a two-component column vector or a $2 \times 2$ matrix, and where

$$
\begin{aligned}
& \mathrm{Z}=\mathrm{e}^{\sigma_{3} \log z}=\left(\begin{array}{cc}
z & 0 \\
0 & 1 / z
\end{array}\right), \quad \mathrm{Q}_{n}(t)=\left(\begin{array}{cc}
0 & q_{n} \\
p_{n} & 0
\end{array}\right), \\
& \mathrm{H}_{n}(z, t)=i \sigma_{3}\left(\mathrm{Q}_{n} \mathrm{Z}^{-1}-\mathrm{Q}_{n-1} \mathrm{Z}-\mathrm{Q}_{n} \mathrm{Q}_{n-1}\right)=i\left(\begin{array}{cc}
-q_{n} p_{n-1} & z q_{n}-q_{n-1} / z \\
z p_{n-1}-p_{n} / z & p_{n} q_{n-1}
\end{array}\right) .
\end{aligned}
$$

In sections 3 and 5 we make frequent use of the integrating factors

$$
\begin{aligned}
& \hat{\mathrm{Z}}(\mathrm{A})=\mathrm{ZAZ}^{-1}=\left(\begin{array}{cc}
a_{11} & z^{2} a_{12} \\
a_{21} / z^{2} & a_{22}
\end{array}\right), \quad \hat{\sigma}_{3} \mathrm{~A}=\left(\begin{array}{cc}
a_{11} & -a_{12} \\
-a_{21} & a_{22}
\end{array}\right), \\
& \mathrm{e}^{i \theta \hat{\sigma}_{3}}(\mathrm{~A})=\mathrm{e}^{i \theta \sigma_{3}} \mathrm{Ae}^{-i \theta \sigma_{3}}=\left(\begin{array}{cc}
a_{11} & \mathrm{e}^{2 i \theta} a_{12} \\
\mathrm{e}^{-2 i \theta} a_{21} & a_{22}
\end{array}\right) .
\end{aligned}
$$

For any matrix $\mathrm{A}$, we write $\mathrm{A}=\left(\mathrm{A}^{(L)}, \mathrm{A}^{(R)}\right)$, where the superscripts $L$ and $R$ (left and right) denote respectively the first and second column of $A$. We also write $A=A_{D}+A_{O}$, where $A_{D}$ and $\mathrm{A}_{O}$ denote respectively the diagonal and off-diagonal part of $\mathrm{A}$. Note that

$$
\begin{aligned}
& (\mathrm{A} \mu)_{D}=\mathrm{A}_{D} \mu_{D}+\mathrm{A}_{O} \mu_{O}, \quad(\mathrm{~A} \mu)_{O}=\mathrm{A}_{O} \mu_{D}+\mathrm{A}_{D} \mu_{O}, \\
& (\mathrm{Q} \mu)_{D}=\mathrm{Q} \mu_{O}, \quad(\mathrm{Q} \mu)_{O}=\mathrm{Q} \mu_{D},
\end{aligned}
$$

and in particular

$$
\mathrm{H}_{n, D}(z, t)=-i \sigma_{3} \mathrm{Q}_{n} \mathrm{Q}_{n-1}, \quad \mathrm{H}_{n, O}(z, t)=i\left(\mathrm{Z} \sigma_{3} \mathrm{Q}_{n}+\mathrm{Q}_{n-1} \mathrm{Z} \sigma_{3}\right) .
$$

Note also that $Z \mathrm{~A}_{O}=\mathbf{A}_{O} \mathbf{Z}^{-1}$ and $\sigma_{3} \mathbf{A}_{O}=-\mathbf{A}_{O} \sigma_{3}$.

The "involution symmetry" of the scattering problems of NLS and IDNLS is expressed through the matrix

$$
\sigma_{v}=\left(\begin{array}{ll}
0 & 1 \\
v & 0
\end{array}\right) .
$$

That is, when $p(x, t)=v q^{*}(x, t)$ in $(\mathrm{A} .3 a)$, or $p_{n}(t)=v q_{n}^{*}(t)$ in (A.6a), it is, respectively:

$$
\sigma_{\nu} \mathrm{Q}^{*}=\mathrm{Q} \sigma_{v}, \quad \sigma_{\nu} \mathrm{Q}_{n}^{*}=\mathrm{Q}_{n} \sigma_{v} .
$$

Note also that $\sigma_{v} \mathbf{Z}=\mathbf{Z}^{-1} \sigma_{v}, \sigma_{\nu} \sigma_{3}=-\sigma_{3} \sigma_{v}$, and $\sigma_{v}^{-1}=\sigma_{v}^{t}=v \sigma_{v}$.

When discussing the asymptotic behavior of the eigenfunctions, the behavior of the matrix product $A Z$ motivates the following definitions: for any matrix $A=\left(A^{(L)}, A^{(R)}\right)$, we write $\mathrm{A}=O\left(\mathrm{Z}^{m}\right)$ as $z \rightarrow(0, \infty)$ if $\mathrm{A}^{(L)}=O\left(z^{m}\right)$ as $z \rightarrow 0$ and $\mathrm{A}^{(R)}=O\left(1 / z^{m}\right)$ as $z \rightarrow \infty$. Similarly, we write $\mathrm{A}=O\left(\mathrm{Z}^{m}\right)$ as $z \rightarrow(\infty, 0)$ if $\mathrm{A}^{(L)}=O\left(z^{m}\right)$ as $z \rightarrow \infty$ and $\mathrm{A}^{(R)}=O\left(1 / z^{m}\right)$ as $z \rightarrow 0$.

\section{Appendix B. Spectral analysis of the $t$-part of the Lax pair of the DLS}

The inversion formulae for the spectral functions (2.14) in the linear problem can be obtained by performing spectral anlaysis of the individual parts of the Lax pair (2.6). The first of (2.17) 
can be derived from similar steps as in section 2.2. As for the second of (2.17), consider the following spectral problem

$$
\mu_{t}+i \omega(z) \mu=f(t)
$$

where $\omega(z)=2-(z+1 / z)$. The Jost solutions are easily obtained, and are:

$$
\mu^{(1)}(z, t)=\int_{0}^{t} \mathrm{e}^{-i \omega(z)\left(t-t^{\prime}\right)} f\left(t^{\prime}\right) \mathrm{d} t^{\prime}, \quad \mu^{(2)}(z, t)=-\int_{t}^{T} \mathrm{e}^{-i \omega(z)\left(t-t^{\prime}\right)} f\left(t^{\prime}\right) \mathrm{d} t^{\prime} .
$$

Note that $\mu^{(1)}$ and $\mu^{(2)}$ are analytic for $z \notin D_{+}$and $z \in D_{+}$, respectively, where $D_{+}$is the same as in section 2.3. Also, the jump condition is

$$
\mu^{(1)}-\mu^{(2)}=\mathrm{e}^{-i \omega(z) t} \hat{f}(z, T), \quad z \in \partial D_{+},
$$

where

$$
\hat{f}(z, t)=\int_{0}^{t} \mathrm{e}^{i \omega(z) t^{\prime}} f\left(t^{\prime}\right) \mathrm{d} t^{\prime} .
$$

Using integration by parts, one can show that $\mu^{ \pm}=O(1 / z)$ as $z \rightarrow \infty$ in their corresponding domains. Hence the solution of the RHP (B.2) is given by

$$
\mu(z, t)=\frac{1}{2 \pi i} \int_{\partial D_{+}} \frac{\mathrm{e}^{-i \omega(\zeta) t} \hat{f}(\zeta, T)}{\zeta-z} \mathrm{~d} \zeta
$$

Substituting this into (B.1), we then find the reconstruction formula

$$
f(t)=-\frac{1}{2 \pi i} \int_{\partial D_{+}} \frac{\omega(\zeta)-\omega(z)}{\zeta-z} \mathrm{e}^{-i \omega(\zeta) t} \hat{f}(\zeta, T) \mathrm{d} \zeta
$$

Recall that $\partial D_{+}=\partial D_{+ \text {in }} \cup \partial D_{+ \text {out }}$. Also note that $\partial D_{+ \text {in }}$ can be deformed to $\partial D_{+ \text {out }}$ by letting $z \rightarrow 1 / z$, and $\omega(z)$ and $\hat{f}(z, t)$ are invariant under this transformation. After some algebra, we then obtain

$$
f(t)=\frac{1}{2 \pi} \int_{\partial D_{\text {+out }}}\left(\frac{1}{z^{2}}-1\right) \mathrm{e}^{-i \omega(z) t} \hat{f}(z, T) \mathrm{d} z .
$$

Replacing $f(z, t)$ by $q_{n}(t)$, we finally obtain the second of (2.17).

Both of (2.17) could also be obtained by more direct methods. The first of (2.17) of course just defines the coefficients of the principal part in the Laurent expansion of $\hat{q}(z, t)$. As for the second of (2.17), it can be obtained as follows. Define $\tilde{q}(t)$ to be the function which equals $q_{n}(t)$ for $0 \leq t \leq T$ and is 0 otherwise. Also, let $\tilde{Q}(\omega)=\int_{-\infty}^{\infty} \mathrm{e}^{i \omega t} \tilde{q}_{n}(t) \mathrm{d} t$ be its Fourier transform. Then, for all $0<t<T$ it is $q_{n}(t)=(1 / 2 \pi) \int_{-\infty}^{\infty} \mathrm{e}^{-i \omega t} \tilde{Q}(\omega) \mathrm{d} \omega$. Note however that the transformation $z \rightarrow \omega(z)$ maps $\partial D_{+ \text {out }}$ onto the real $\omega$-axis, with $\omega(z)$ decreasing monotonically as $\operatorname{Re} z$ increases. Moreover, $\tilde{Q}(\omega(z))=\hat{f}_{n}(z, T)$. Hence we can rewrite the previous integral as $q_{n}(t)=(1 / 2 \pi) \int_{\partial D_{\text {+out }}} \omega^{\prime}(z) \mathrm{e}^{-i \omega(z) t} \hat{f}_{n}(z, T) \mathrm{d} z$.

\section{Appendix C. IBVPs for DLS with Robin-type boundary conditions}

Consider the DLS equation (1.1) for $n \in \mathbb{N}_{0}$ and $t \in \mathbb{R}^{+}$with mixed BCs. The spectral transform of (2.27) yields, $\forall z \in \mathbb{C}^{[\emptyset]}$,

$$
\hat{f}_{-1}(z, t)-\alpha \hat{f}_{0}(z, t)=\hat{h}(z, t),
$$

where the $\hat{f}_{j}(z, t)$ are given by $(2.14)$, and $\hat{h}(z, t)$ is defined similarly. Recall that the reconstruction formula (2.19) contains the quantity $\hat{F}(z, t)=i\left(z \hat{f}_{0}(z, t)-\hat{f}_{-1}(z, t)\right)$. Use of (C.1) 
and the transformed global relation (2.23) allows one to eliminate $\hat{f}_{0}(z, t)$ and $\hat{f}_{-1}(z, t)$ and express $\hat{F}(z, t)$, for all $0<|z| \leq 1$, as

$$
\hat{F}(z, t)=\frac{\hat{G}(z, t)}{1 / z-\alpha}-\frac{z-\alpha}{1 / z-\alpha} \mathrm{e}^{i \omega(z) t} \hat{q}(1 / z, t),
$$

where $\hat{G}(z, t)$, which contains the known portion of the RHS, was given in (2.29). Now recall that, in (2.19), $\hat{F}(z, t)$ is integrated along $\partial D_{+ \text {in }}$. Three possible situations can arise: (i) $\alpha \in D_{+ \text {out }}$, (ii) $\alpha \in \partial D_{+ \text {out }}$, (iii) $\alpha \notin \bar{D}_{+ \text {out }}$. We discuss each of these cases in turn.

If $\alpha \notin \bar{D}_{\text {+out }}$, the denominator of (C.2) never vanishes in $\bar{D}_{\text {+in }}$. Thus the second part of the RHS of (C.2), when inserted in (2.19), gives rise to an integrand that is analytic and bounded in $\bar{D}_{+ \text {in }}$. Hence, that part of the integral is zero. As a result, the solution of the IBVP is simply

$q_{n}(t)=\frac{1}{2 \pi i} \oint_{|z|=1} z^{n-1} \mathrm{e}^{-i \omega(z) t} \hat{q}(z, 0) \mathrm{d} z-\frac{1}{2 \pi i} \int_{\partial D_{+ \text {in }}} z^{n-1} \mathrm{e}^{-i \omega(z) t} \frac{\hat{G}(z, T)}{1 / z-\alpha} \mathrm{d} z$,

with $\hat{G}(z, t)$ again given by (2.29). Now suppose $\alpha \in D_{+ \text {out }}$. In this case $1 / z-\alpha$ vanishes at $z=1 / \alpha \in D_{+ \text {in }}$. Even though each of the two terms in the RHS of (C.2) has a simple pole at this point, however, their sum is finite there, since $\hat{F}(z, t)$ is analytic in $\mathbb{C}^{[\emptyset]}$. Thus,

$$
\begin{aligned}
& \frac{1}{2 \pi i} \int_{\partial D_{+ \text {in }}} z^{n-1} \frac{z-\alpha}{1 / z-\alpha} \hat{q}(1 / z, t) \mathrm{d} z=\operatorname{Res}_{z=1 / \alpha} {\left[z^{n-1} \frac{z-\alpha}{1 / z-\alpha} \hat{q}(1 / z, t)\right] } \\
&=\operatorname{Res}_{z=1 / \alpha}\left[z^{n-1} \mathrm{e}^{-i \omega(z) t} \frac{\hat{G}(z, t)}{1 / z-\alpha}\right]=-\alpha^{-n-1} \mathrm{e}^{-i \omega(\alpha) t} \hat{G}(1 / \alpha, t),
\end{aligned}
$$

which implies the solution of the IBVP as

$q_{n}(t)=\frac{1}{2 \pi i} \oint_{|z|=1} z^{n-1} \mathrm{e}^{-i \omega(z) t} \hat{q}(z, 0) \mathrm{d} z-\frac{1}{2 \pi i} \int_{\partial D_{+ \text {in }}} z^{n-1} \mathrm{e}^{-i \omega(z) t} \frac{\hat{G}(z, T)}{1 / z-\alpha} \mathrm{d} z-\alpha^{-n-1} \mathrm{e}^{-i \omega(\alpha) t} \hat{G}(1 / \alpha, t)$.

Finally, if $\alpha \in \partial D_{+ \text {out }}$, the pole is along the integration contour. In this case one should go back to the RHP and subtract the pole contribution. In this way, the solution of the IBVP can be obtained as

$q_{n}(t)=\frac{1}{2 \pi i} \oint_{|z|=1} z^{n-1} \mathrm{e}^{-i \omega(z) t} \hat{q}(z, 0) \mathrm{d} z-\frac{1}{2 \pi i} \int_{\partial D_{+ \text {in }}} z^{n-1} \mathrm{e}^{-i \omega(z) t} \frac{\hat{G}(z, T)}{1 / z-\alpha} \mathrm{d} z-\frac{1}{2} \alpha^{-n-1} \mathrm{e}^{-i \omega(\alpha) t} \hat{G}(1 / \alpha, t)$.

Combining (C.3), (C.4) and (C.5) one then obtains (2.28).

\section{Appendix D. Asymptotic behavior of the eigenfunctions of the IBVP}

$D L S$. We first compute the asymptotics for for $n=0$ (where no summation is present), then consider the case $n \geq 1$. Note that $\omega(z)=-1 / z+O\left(1 / z^{2}\right)$ as $z \rightarrow 0$. Integration by parts yields, as $z \rightarrow 0$ with $\operatorname{Im} z \leq 0$,

$$
\phi_{0}^{(1)}(z, t)=q_{-1}(t)-\mathrm{e}^{-i \omega(z) t} q_{-1}(0)+O(z),
$$

while as $z \rightarrow 0$ with $\operatorname{Im} z \geq 0$ it is

$$
\phi_{0}^{(3)}(z, t)=q_{-1}(t)-\mathrm{e}^{i \omega(z)(t-T)} q_{-1}(T)+O(z) .
$$

Using these in (2.13) with $n \geq 1$ we then have immediately $\phi_{n}^{(j)}(z, t)=q_{n-1}(t)+O(z)$ as $z \rightarrow 0$ with $\operatorname{Im} z \leq 0$ for $j=1$ and $\operatorname{Im} z \geq 0$ for $j=3$. Note also that $\phi_{0}^{(1)}(z, t)-\phi_{0}^{(3)}(z, t)=$ $-\mathrm{e}^{-i \omega(z) t}\left(q_{-1}(0)-\mathrm{e}^{i \omega(z) T} q_{-1}(T)\right)+O(z)$ as $z \rightarrow 0$, implying that the ratio $\hat{F}(z, T) / z$ in $(2.14 b)$ remains bounded as $z \rightarrow 0$ along the real axis. As for $\phi_{n}^{(2)}(z, t),(2.9)$ implies immediately $\phi_{n}^{(2)}(z, t)=O(1 / z)$ as $z \rightarrow \infty$. 
IDNLS. The determination of the asymptotic behavior in the nonlinear case is considerably more involved, and requires the use of a Neumann series approach:

$$
\mu_{n}^{(j)}(z, t)=\sum_{m=0}^{\infty} \mu_{n}^{(j, m)}(z, t) .
$$

We now show that, $\forall n \in \mathbb{N}_{0}, m \geq 0$ and $j=1,3$, as $z \rightarrow(\infty, 0)$ it is

$$
\begin{aligned}
& \mu_{n, D}^{(j, 2 m-1)}(z, t)=O\left(\mathrm{Z}^{-2 m}\right), \quad \mu_{n, O}^{(j, 2 m-1)}(z, t)=O\left(\mathrm{Z}^{-2 m+1}\right), \\
& \mu_{n, D}^{(j, 2 m)}(z, t)=O\left(\mathrm{Z}^{-2 m}\right), \quad \mu_{n, O}^{(j, 2 m)}(z, t)=O\left(\mathrm{Z}^{-2 m-1}\right) .
\end{aligned}
$$

The proof proceeds by induction. Consider $\mu_{n}^{(1)}(z, t)$ first. Separating (3.25a) into its diagonal and off-diagonal components then yields $\mu_{n, D}^{(1,0)}(z, t)=\mathrm{I}$ and $\mu_{n, O}^{(1,0)}(z, t)=\mathrm{O}$, as well as

$$
\begin{aligned}
\mu_{n, D}^{(1, m+1)}(z, t) & =\sum_{n^{\prime}=0}^{n-1} \mathrm{Q}_{n^{\prime}}(t) \mu_{n^{\prime}, O}^{(1, m+1)}(z, t) \mathrm{Z}^{-1}+\int_{0}^{t}\left(\mathrm{H}_{0, D} \mu_{0, D}^{(1, m)}+\mathrm{H}_{0, O} \mu_{0, O}^{(1, m+1)}\right)\left(z, t^{\prime}\right) \mathrm{d} t^{\prime}, \\
\mu_{n, O}^{(1, m+1)}(z, t)= & \sum_{n^{\prime}=0}^{n-1} \mathrm{Q}_{n^{\prime}}(t) \mu_{n^{\prime}, D}^{(1, m)}(z, t) \mathrm{Z}^{-2\left(n-n^{\prime}\right)+1} \\
& \quad \int_{0}^{t} \mathrm{e}^{-i \omega(z)\left(t-t^{\prime}\right) \hat{\sigma}_{3}}\left(\mathrm{H}_{0, O} \mu_{0, D}^{(1, m)}+\mathrm{H}_{0, D} \mu_{0, O}^{(1, m)}\right)\left(z, t^{\prime}\right) \mathrm{Z}^{-2 n} \mathrm{~d} t^{\prime} .
\end{aligned}
$$

Note that

$$
\frac{1}{2 \omega(z)} \mathrm{I}=-\mathrm{Z}^{-2}+O\left(\mathrm{Z}^{-4}\right), \quad \text { as } z \rightarrow(\infty, 0) .
$$

First consider the case $n=0$. Using integration by parts in (D.3b), we obtain, as $z \rightarrow(\infty, 0)$,

$$
\begin{aligned}
\mu_{0, O}^{(1, m+1)}(z, t)=\left\{\mathrm{Q}_{-1}(t) \mu_{0, D}^{(1, m)}(z, t)-\mathrm{e}^{-i \omega(z) t \hat{\sigma}_{3}}\left[\mathrm{Q}_{-1}(0) \mu_{0, D}^{(1, m)}(z, 0)\right]\right\} \mathrm{Z}^{-1} \\
+ \\
+\left\{\left(\mathrm{Q}_{0} \mathrm{Q}_{-1}\right)(t) \mu_{0, O}^{(1, m)}(z, t)-\mathrm{e}^{-i \omega(z) t \hat{\sigma}_{3}}\left[\left(\mathrm{Q}_{0} \mathrm{Q}_{-1}\right)(0) \mu_{0, O}^{(1, m)}(z, 0)\right]\right\} \mathrm{Z}^{-2},
\end{aligned}
$$

plus higher order terms. Substituting (D.4a) into (D.3a) with $n=0$, one finds

$$
\begin{gathered}
\mu_{0, D}^{(1, m+1)}(z, t)=-i \int_{0}^{t} \sigma_{3}\left(\mathrm{Q}_{0} \mathrm{Q}_{-1}\right)\left(t^{\prime}\right) \mu_{0, D}^{(1, m)}\left(z, t^{\prime}\right) \mathrm{d} t^{\prime}+i \int_{0}^{t} \sigma_{3} \mathrm{Q}_{0}\left(t^{\prime}\right) \mu_{0, O}^{(1, m+1)}\left(z, t^{\prime}\right) \mathrm{Z} t^{\prime} \\
-i \int_{0}^{t} \sigma_{3} \mathrm{Q}_{-1}\left(t^{\prime}\right) \mu_{0, O}^{(1, m+1)}\left(z, t^{\prime}\right) \mathrm{Z}^{-1} \mathrm{~d} t^{\prime} .
\end{gathered}
$$

Using (D.4) one can then obtain (D.2) for $n=0$ and all $m \in \mathbb{N}_{0}$ inductively. Note also that, for $m=0$, (D.4a) yields (3.32b). Similarly, repeating the same arguments, one obtains (3.32c).

Next consider the case $n \geq 1$. The integrals in (D.3) are exactly the same as when $n=0$ except for the fact that the one in (D.3b) is followed by $Z^{-2 n}$. Using the same arguments as before, we obtain

$$
\begin{aligned}
& \mu_{n, O}^{(1, m+1)}(z, t)=\mathrm{Q}_{n-1}(t) \mu_{n-1, D}^{(1, m)}(z, t) \mathrm{Z}^{-1}+\mu_{0, O}^{(1, m+1)}(z, t) \mathrm{Z}^{-2 n}+\cdots, \\
& \mu_{n, D}^{(1, m+1)}(z, t)=\sum_{l=0}^{n-1} \mathrm{Q}_{l}(t) \mu_{l, O}^{(1, m+1)}(z, t) \mathrm{Z}^{-1}+\mu_{0, D}^{(1, m+1)}(z, t) .
\end{aligned}
$$

The induction with (D.5), one can derive (D.2) for $n \geq 1$. Similarly, one obtains (D.2) for $\mu_{n}^{(3)}$. This completes the proof of (D.2).

The above results imply that $\mu_{n}^{(1)}(z, t)=\mathrm{I}+O\left(\mathrm{Z}^{-1}\right)$ as $z \rightarrow(\infty, 0)$. In particular, computing the $O\left(Z^{-1}\right)$ terms explicitly one obtains the first of (3.32a). Similarly, using the same arguments, one can show that $\mu_{n}^{(3)}(z, t)=\mathrm{I}+O\left(\mathrm{Z}^{-1}\right)$ as $z \rightarrow(\infty, 0)$ and verify the second 
of (3.32a). In the IVP, the integrals in the RHS of (D.3a) and (D.3b) are absent, and the summation starts from $n^{\prime}=-\infty$. Hence in this case one simply obtains (3.17).

The determination of the asymptotic behavior of $\mu_{n}^{(2)}(z, t)$ requires a slightly different approach, since following the above steps for $\mu_{n}^{(2)}(z, t)$, yields a $O(1)$ term involving the summation of $\mathrm{Q}_{n}$ in the RHS. To circumvent this difficulty, note that (A.5a) implies $\mu_{n}^{(2)}=$ $\left(\mathrm{Z}+\mathrm{Q}_{n}(t)\right)^{-1} \mu_{n+1}^{(2)}$. For $\tilde{\mu}_{n}(z, t)=C_{n} \mu_{n}^{(2)}(z, t)$ we have

$$
\tilde{\mu}_{n}-\hat{Z}^{-1} \tilde{\mu}_{n+1}=-\mathrm{Q}_{n} \tilde{\mu}_{n} \mathrm{Z}
$$

with $\tilde{\mu}_{n}(z, t) \rightarrow I$ as $n \rightarrow \infty$ thanks to (3.25a) and (3.6). Introducing the auxiliary function $\Psi_{n}(z, t)=\hat{Z}^{-n} \tilde{\mu}_{n}(z, t)$, it is easy to check that $\Psi_{n}(z, t)$ satisfies the equation $\Psi_{n+1}-\Psi_{n}=$ $Z \hat{Z}^{-(n+1)}\left(Q_{n}\right) \Psi_{n+1}$, which can be integrated to obtain the modified Jost solution as

$$
\tilde{\mu}_{n}(z, t)=\mathrm{I}-\mathrm{Z} \sum_{n^{\prime}=n+1}^{\infty} \hat{Z}^{n-n^{\prime}}\left(\mathrm{Q}_{n^{\prime}-1}(t) \tilde{\mu}_{n^{\prime}}(z, t)\right) .
$$

Then, applying the same Neumann series approach as described above to (D.7), one finds the asymptotic expansion for $\mu_{n}^{(2)}$ as (3.18). Since $\mu_{n}^{(2)}(z, t)$ is the same in the IVP and in the IBVP; this asymptotic behavior applies to both problems.

Note that the above results also imply that $a(z)$ and $d(z)$ are even functions in $D_{ \pm \text {in }}$ and the following symmetries of $\mathrm{M}_{n}^{ \pm}$:

$$
\begin{array}{ll}
\mathrm{M}_{n, 11}^{+}(-z, t)=\mathrm{M}_{n, 11}^{+}(z, t), & \mathrm{M}_{n, 12}^{+}(-z, t)=-\mathrm{M}_{n, 12}^{+}(z, t), \\
\mathrm{M}_{n, 11}^{-}(-z, t)=\mathrm{M}_{n, 11}^{-}(z, t), & \mathrm{M}_{n, 12}^{-}(-z, t)=-\mathrm{M}_{n, 12}^{-}(-z, t) .
\end{array}
$$

\section{Appendix E. Independence of the solution on $T$}

The solution of a DDE does not depend on future values of the BCs. Hence, for any $T_{0}<T$ the solution of the IBVP resulting from the RHP obtained by replacing $T$ with $T_{0}$ must be equivalent for all $0<t<T_{0}$ to the solution of the IBVP obtained from the original RHP. We show next that is indeed the case because the RHP obtained from $T_{0}$ and $T$ are related.

Let $\mathrm{M}_{n}(z, t)$ satisfy the RHP (3.36), and let $\mathrm{M}_{n}^{ \pm \text {in }}(z, t)$ and $\mathrm{M}_{n}^{ \pm \text {out }}(z, t)$ denote the restrictions of $\mathrm{M}_{n}(z, t)$ to the domains $D_{ \pm \text {in }}$ and $D_{ \pm \text {out }}$, respectively. Moreover, let $A\left(z, T_{0}\right)$ and $B\left(z, T_{0}\right)$ be the spectral coefficients obtained by replacing $T$ with $T_{0}$ in (E.3), and let $\tilde{J}_{n}^{(1)}(z, t), \ldots, \tilde{J}_{n}^{(4)}(z, t)$ denote the jump matrices obtained by replacing $A(z, T)$ and $B(z, T)$ with $A\left(z, T_{0}\right)$ and $B\left(z, T_{0}\right)$. Finally, let $\tilde{\mathrm{M}}_{n}(z, t)$ satisfy the RHP with the jump matrices $J_{n}^{(1)}, \ldots, J_{n}^{(4)}$ replaced by $\widetilde{J}_{n}^{(1)}, \ldots, \widetilde{J}_{n}^{(4)}$. It is straightforward to see the relations

$$
\begin{aligned}
& \mathrm{M}_{n}^{+ \text {in }}=\tilde{\mathrm{M}}_{n}^{+ \text {in }}\left(\mathrm{I}-\tilde{\mathrm{J}}_{n}^{(1)}\right)\left(\mathrm{I}-\mathrm{J}_{n}^{(1)}\right)^{-1}, \quad \mathrm{M}_{n}^{- \text {in }}=\tilde{\mathrm{M}}_{n}^{- \text {in }}, \\
& \mathrm{M}_{n}^{\text {+out }}=\tilde{\mathrm{M}}_{n}^{\text {+out }}, \quad \mathrm{M}_{n}^{\text {-out }}=\tilde{\mathrm{M}}_{n}^{\text {-out }}\left(\mathrm{I}-\tilde{\mathrm{J}}_{n}^{(3)}\right)^{-1}\left(\mathrm{I}-\mathrm{J}_{n}^{(3)}\right) .
\end{aligned}
$$

Now recall that $q_{n}(t)$ can be obtained from the eigenfunctions via (3.40) or (3.32a) with $j=1$. Note also that $\mu_{n}^{(1, R)}(z, t)$ enters $\mathrm{M}_{n}^{- \text {in }}$ via $(3.35 b)$. Below, we show that the matrices $\left(\mathrm{I}-\tilde{\mathrm{J}}_{n}^{(1)}\right)\left(\mathrm{I}-\mathrm{J}_{n}^{(1)}\right)^{-1}$ and $\left(\mathrm{I}-\tilde{\mathrm{J}}_{n}^{(3)}\right)^{-1}\left(\mathrm{I}-\mathrm{J}_{n}^{(3)}\right)$ are analytic and bounded for $z \in D_{+ \text {in }}$ and $z \in D_{\text {+out }}$, respectively. Since $\mathrm{M}_{n}(z, t)=\tilde{\mathrm{M}}_{n}(z, t)$ for $z \in D_{- \text {in }}$, it then follows that the solutions $q_{n}(t)$ obtained from $\mathrm{M}_{n}$ and $\tilde{\mathrm{M}}_{n}$ coincide.

To show that $\left(\mathrm{I}-\widetilde{J}_{n}^{(1)}\right)\left(\mathrm{I}-\mathrm{J}_{n}^{(1)}\right)^{-1}$ is analytic and bounded for $z \in D_{+ \text {in }}$, note first that

$$
\left(\mathrm{I}-\tilde{\mathrm{J}}_{n}^{(1)}\right)\left(\mathrm{I}-\mathrm{J}_{n}^{(1)}\right)^{-1}=\left(\begin{array}{cc}
1 & v z^{2 n} \mathrm{e}^{-2 i \omega(z) t}\left(\Gamma^{*}\left(1 / z^{*}, T\right)-\Gamma^{*}\left(1 / z^{*}, T_{0}\right)\right) \\
0 & 1
\end{array}\right),
$$

and the $(1,2)$ component of (E.1) can be written as

$$
X_{n}(z)=v z^{2 n} \mathrm{e}^{-2 i \omega(z) t} \frac{A^{*}\left(1 / z^{*}, T_{0}\right) B^{*}\left(1 / z^{*}, T\right)-A^{*}\left(1 / z^{*}, T\right) B^{*}\left(1 / z^{*}, T_{0}\right)}{d(z, T) d\left(z, T_{0}\right)} .
$$


Now note that (3.28) and (3.31) define the scattering data $A(z, T)$ and $B(z, T)$ as

$$
\mu_{0}^{(1, R)}(z, T)=\left(\begin{array}{c}
-v \mathrm{e}^{-2 i \omega(z) T} B^{*}\left(1 / z^{*}, T\right) \\
A(z, T)
\end{array}\right)=:\left(\begin{array}{c}
\mu_{1}(z, T) \\
\mu_{2}(z, T)
\end{array}\right),
$$

Hence

$$
X_{n}(z)=z^{2 n} \mathrm{e}^{2 i \omega(z)\left(T_{0}-t\right)} \frac{\mu_{2}^{*}\left(1 / z^{*}, T\right) \mu_{1}\left(z, T_{0}\right)-\mu_{2}^{*}\left(1 / z^{*}, T_{0}\right) \mu_{1}(z, T) \mathrm{e}^{2 i \omega(z)\left(T-T_{0}\right)}}{d(z, T) d\left(z, T_{0}\right)} .
$$

Also, $\mu_{0}^{(1, R)}(z, t)$ satisfies the second column of the $t$-part of the Lax pair (A.5) at $n=0$ :

$$
\begin{aligned}
& \dot{\mu}_{1}(z, t)+2 i \omega(z) \mu_{1}(z, t)=H_{0,11}(z, t) \mu_{1}(z, t)+H_{0,12}(z, t) \mu_{2}(z, t), \\
& \dot{\mu}_{2}(z, t)=H_{0,21}(z, t) \mu_{1}(t, k)+H_{0,22}(z, t) \mu_{2}(z, t) .
\end{aligned}
$$

Then, introducing

$$
\begin{aligned}
& \varphi_{1}(z, t)=\mu_{2}^{*}\left(1 / z^{*}, T\right) \mu_{1}(z, t)-\mu_{1}(z, T) \mu_{2}^{*}\left(1 / z^{*}, t\right) \mathrm{e}^{2 i \omega(z)(T-t)}, \\
& \varphi_{2}(z, t)=\mu_{2}^{*}\left(1 / z^{*}, T\right) \mu_{2}(z, t)-v \mu_{1}(z, T) \mu_{1}^{*}\left(1 / z^{*}, t\right) \mathrm{e}^{2 i \omega(z)(T-t)},
\end{aligned}
$$

we can rewrite the $(1,2)$ component of $\left(I-\tilde{J}_{n}^{(1)}\right)\left(I-J_{n}^{(1)}\right)^{-1}$ as

$$
X_{n}(z)=\frac{z^{2 n} \mathrm{e}^{2 i \omega(z)\left(T_{0}-t\right)}}{d(z, T) d\left(z, T_{0}\right)} \varphi_{1}\left(z, T_{0}\right)
$$

It is therefore enough to show that $\varphi_{1}(z, t)$ is analytic and bounded for $z \in D_{+}$. The symmetries of $\mathrm{H}_{0}(z, t)$ [namely, $\mathrm{H}_{0,12}(z, t)=v \mathrm{H}_{0,21}^{*}\left(1 / z^{*}, t\right)$ and $\mathrm{H}_{0,11}(z, t)=\mathrm{H}_{0,22}\left(1 / z^{*}, t\right)$ ] imply that $\left(\varphi_{1}, \varphi_{2}\right)^{t}$ satisfies the $t$-part of the Lax pair (A.5b) with $n=0$. Since $\varphi_{1}(z, T)=0$ and $\varphi_{2}(z, T)=1$, we then have the following linear integral equations

$$
\begin{aligned}
& \varphi_{1}(z, t)=-\int_{t}^{T} \mathrm{e}^{2 i \omega(z)\left(t^{\prime}-t\right)}\left(\mathrm{H}_{0,11} \varphi_{1}+\mathrm{H}_{0,12} \varphi_{2}\right)\left(z, t^{\prime}\right) \mathrm{d} t^{\prime}, \\
& \varphi_{2}(z, t)=1-\int_{t}^{T}\left(\mathrm{H}_{0,21} \varphi_{1}+\mathrm{H}_{0,22} \varphi_{2}\right)\left(z, t^{\prime}\right) \mathrm{d} t^{\prime},
\end{aligned}
$$

From here one can show that $\varphi_{1}$ and $\varphi_{2}$ are analytic and bounded for $z \in D_{+}$. As a result, the RHS of (E.2) is analytic and bounded for $z \in D_{+}$. Thus $\left(I-\tilde{J}_{n}^{(1)}\right)\left(I-J_{n}^{(1)}\right)^{-1}$ is analytic and bounded for $z \in D_{+ \text {in }}$. The result for $\left(I-\tilde{J}_{n}^{(3)}\right)^{-1}\left(I-J_{n}^{(3)}\right)$ follows from symmetry considerations.

\section{Appendix F. Linearizable BCs for $T<\infty$}

Here we verify that (4.10) can be used to express $\Gamma^{*}\left(1 / z^{*}\right)$ also when $T<\infty$. To do so, we use the same approach that we used to show that the solution of the IDNLS equation does not depend on $T$. Denote by $X_{n}(z)$ the difference between the contributions to the RHP obtained from $T=\infty$ and $T<\infty$, namely:

$$
X_{n}(z)=v z^{2 n} \mathrm{e}^{-2 i \omega(z) t}\left(\Gamma^{*}\left(1 / z^{*}\right)-\Gamma_{o}^{*}\left(1 / z^{*}\right)\right),
$$

where $\Gamma_{o}^{*}\left(1 / z^{*}\right)$ is obtained by neglecting the second term in the RHS of (4.11). We can write (F.1) as

$$
X_{n}(z)=v z^{2 n} \mathrm{e}^{-2 i \omega(z) t} \frac{R(z, T)-R_{o}(z, T)}{d(z) d_{o}(z) / A^{*}\left(1 / z^{*}, T\right) A_{o}^{*}\left(1 / z^{*}, T\right)},
$$

with $R(z, T)=B^{*}\left(1 / z^{*}, T\right) / A^{*}\left(1 / z^{*}, T\right)$ as before, and where $R_{o}(z)=B_{o}^{*}\left(1 / z^{*}\right) / A_{o}^{*}\left(1 / z^{*}\right)$ is computed using only the first term in the RHS of (4.11) and $d_{o}(z)=a(z) A_{o}^{*}\left(1 / z^{*}, T\right)-$ 
$v b(z) B_{o}^{*}\left(1 / z^{*}, T\right)$. Also, $A_{o}^{*}\left(1 / z^{*}, T\right)$ and $B_{o}^{*}\left(1 / z^{*}, T\right)$ are defined by (4.9). Now, using (4.11), we find

$$
X_{n}(z)=z^{2 n} \mathrm{e}^{2 i \omega(z)(T-t)} f(1 / z) \frac{G(1 / z, T)}{d(z) \Delta(1 / z)} .
$$

In the solitonless case, however, we can assume that $d(z)$ and $\Delta(1 / z)$ never vanish in $\bar{D}_{\text {+in }}$. Then the RHS of (F.2) is analytic and bounded in $D_{+ \text {in }}$ due to the exponential term and now we know that the additional term in (4.11) does not affect the solution of the RHP. Note that $f(1 / z)$ has a pole at $z= \pm 1 / \chi^{1 / 2}$. When $1<\chi$, or $\chi<-1$, these points belong to $D_{+ \text {in }}$. Note, however, that since $a(z)$ and $b(z)$ are bounded in $\bar{D}_{ \pm \text {in }}$, if $f(1 / z)$ has a pole, $\Delta(1 / z)$ does too, and hence the terms causing the poles in (F.2) to cancel out.

\section{References}

[1] M J Ablowitz, G Biondini and B Prinari, "Inverse scattering transform for the integrable discrete nonlinear Schrödinger with non-vanishing boundary conditions”, Inv. Probl. 23, 1711-1758 (2007)

[2] M J Ablowitz and P A Clarkson, Solitons, nonlinear evolution equations and inverse scattering (Cambridge University Press, Cambridge, 1991)

[3] M J Ablowitz, R G Halburd and B Herbst, "On the extension of the Painleve property to difference equations", Nonlinearity 13, 889-905 (2000)

[4] M J Ablowitz and J F Ladik, "Nonlinear differential-difference equations", J. Math. Phys. 16, 598 (1975)

[5] M J Ablowitz and J F Ladik, "Nonlinear differential-difference equations and Fourier analysis", J. Math. Phys. 17, 1011 (1976)

[6] M J Ablowitz, B Prinari and A D Trubatch, Discrete and continuous nonlinear Schrödinger systems, London Mathematical Society Lecture Note Series 302 (Cambridge University Press, 2003)

[7] M J Ablowitz and H Segur, Solitons and the inverse scattering transform (SIAM, Philadelphia, 1981)

[8] M J Ablowitz and H Segur, "The inverse scattering transform: semi-infinite interval", J. Math. Phys. 16, 1054-1056 (1975)

[9] V Adler, B Gurel, M Gurses and I Habibullin, "Boundary conditions for integrable equations", $J$. Phys. A 30, 3505-3513 (1997)

[10] E. D. Belokolos, A. I. Bobenko, V. Z. Enolskii, A. R. Its and V. B. Matveev, Algebro-geometric approach to nonlinear integrable equations (Springer, New York, 1994)

[11] R F Bikbaev and V O Tarasov, "Initial-boundary value problem for the nonlinear Schrödinger equation", J. Phys. A 24, 2507-2516 (1991)

[12] A Boutet de Monvel and V Kotlyarov, "Scattering problem for the Zakharov-Shabat equations on the semi-axis", Inv. Probl. 16, 1813-1837 (2000)

[13] F Calogero and S De Lillo, "The Burgers equation on semi-infinite and infinite intervals", Nonlinearity 2, 37 (1989)

[14] F Calogero and S De Lillo, "The Burgers equation on the semiline with general boundary conditions at the origin", J. Math. Phys. 32, 99 (1991)

[15] A Degasperis, S V Manakov and P M Santini, "On the initial-boundary value problems for soliton equations", JETP Lett. 74, 481-485 (2001)

[16] P Deift, S Venakides and X Zhou, "New results in small dispersion KdV by an extension of the steepest descent method for Riemann-Hilbert problems", Int. Math. Res. Notices 6, 286-299 (1997)

[17] P Deift and X Zhou, "A steepest descent method for oscillatory Riemann-Hilbert problems", Bull. Amer. Math. Soc. 26, 119-123 (1992)

[18] L Ehrenpreis, Fourier analysis in several complex variables (Wiley, New York, 1970) 
[19] L D Faddeev and L A Takhtajan, Hamiltonian Methods in the Theory of Solitons (Springer, Berlin, 1987)

[20] H Flaschka, "On the Toda lattice. 1. Existence of integrals", Phys. Rev. B 9, 1924, (1974)

[21] H Flaschka, "On the Toda lattice. 2. Inverse-scattering solution”, Prog. Theor. Phys. 51, 703 (1974)

[22] A S Fokas, “An initial-boundary value problem for the nonlinear Schrödinger equation”, Phys. $D$ 35, 167-185 (1989)

[23] A S Fokas, "A unified transform method for solving linear and certain nonlinear PDEs", Proc. Roy. Soc. London A 453, 1411-1443 (1997)

[24] A S Fokas, "On the integrability of certain linear and nonlinear partial differential equations", $J$. Math. Phys. 41, 4188-4237 (2000)

[25] A S Fokas, "A new transform method for evolution partial differential equations", IMA J. Appl. Math. 67, 559-590 (2002)

[26] A S Fokas, "Integrable nonlinear evolution equations on the half line", Commun. Math. Phys. 230, 1-39 (2002)

[27] A S Fokas, "Ehrenpreis type representations and their Riemann-Hilbert nonlinearization" $J$. Nonlin. Math. Phys 10, 47-61 (2003)

[28] A S Fokas, "The generalized Dirichlet-to-Neumann map for certain nonlinear evolution PDEs", Commun. Pure Applied Math. 58, 639-670 (2005)

[29] A S Fokas, A R Its and L-Y Sung, "The nonlinear Schrödinger equation on the half-line", Nonlinearity 18, 1771-1822 (2005)

[30] A S Fokas and B Pelloni, "Integral transforms, spectral representations and the D-bar problem", Proc. Roy. Soc. London A 456, 805-833 (2000)

[31] I Habibullin, "Boundary conditions for integrable chains", Phys. Lett. A 207, 263-268 (1995)

[32] G Henkin, "Method of integral representations in complex analysis", Encyclopedia of Mathematical Sciences, Vol. 7: Several complex variables (Springer, Berlin, 1990)

[33] P D Lax, "Integrals of nonlinear equations of evolution and solitary waves" Commun. Pure Appl. Math. 21, 467 (1968)

[34] S V Manakov, "On the theory of two-dimensional stationary self-focusing of electromagnetic waves", Sov. Phys. JETP 38, 248 (1974)

[35] S V Manakov, "Complete integrability and stochastization of discrete dynamical systems", Sov. Phys. JETP 40, 269-274 (1975)

[36] K-i Maruno and G Biondini, "Resonance and web structure in discrete soliton systems: the twodimensional Toda lattice and its fully- and ultra-discrete analogues", J. Phys. A 37, 1181911839 (2004)

[37] V P Palamodov, Linear differential operators with constant coefficients (Springer, Berlin, 1970)

[38] B Prinari, M J Ablowitz and G Biondini, "Inverse scattering transform for the vector nonlinear Schrödinger equation with non-vanishing boundary conditions”, J. Math. Phys. 47, 063508 (2006)

[39] P C Sabatier, "Elbow scattering and inverse scattering: applications to LKdV and KdV", J. Math. Phys. 41, 414-436 (2000)

[40] P C Sabatier, "Generalized inverse scattering transform applied to linear partial differential equations”, Inv. Probl. 22, 209-228 (2006)

[41] E K Skylanin, "Boundary conditions for integrable equations", Funct. Anal. Appl. 21, 86-87 (1987)

[42] M Toda, "Studies of a nonlinear lattice", Phys. Rep. 18, 1-123 (1975)

[43] V E Vekslerchik and V V Konotop, "Discrete nonlinear Schrödinger equation under non-vanishing boundary conditions", Inv. Probl. 8, 889-909 (1992)

[44] V E Zakharov and A B Shabat, "Exact theory of two-dimensional self-focusing and onedimensional self-modulation of waves in nonlinear media", Sov. Phys. JETP, 34, 62 (1972)

[45] V E Zakharov and A B Shabat, "Interaction between solitons in a stable medium", Sov. Phys. JETP 37, 823 (1973) 